TRANSACTIONS OF THE

AMERICAN MATHEMATICAL SOCIETY

Volume 361, Number 4, April 2009, Pages 2109-2161

S 0002-9947(08)04660-6

Article electronically published on November 12, 2008

\title{
DEFORMATIONS OF SINGULARITIES AND VARIATION OF GIT QUOTIENTS
}

\author{
RADU LAZA
}

\begin{abstract}
We study the deformations of the minimally elliptic surface singularity $N_{16}$. A standard argument reduces the study of the deformations of $N_{16}$ to the study of the moduli space of pairs $(C, L)$ consisting of a plane quintic curve and a line. We construct this moduli space in two ways: via the periods of $K 3$ surfaces and by using geometric invariant theory (GIT). The GIT construction depends on the choice of the linearization. In particular, for one choice of linearization we recover the space constructed via $K 3$ surfaces and for another we obtain the full deformation space of $N_{16}$. The two spaces are related by a series of explicit flips. In conclusion, by using the flexibility given by GIT and the standard tools of Hodge theory, we obtain a good understanding of the deformations of $N_{16}$.
\end{abstract}

\section{INTRODUCTION}

Singularities and their deformations have always played a central role in algebraic geometry, being of fundamental importance in several branches of the field, such as the classification of surfaces, the minimal model program, and the compactification problem for moduli spaces. The easiest and the first to be understood were the simple singularities (also known as duVal singularities). The work of many mathematicians, including Brieskorn, Pinkham, and Looijenga, has extended the results for simple singularities to the next level of complexity, the unimodal singularities (simple elliptic, cusp and triangle). The focus of this paper is a detailed study of a new class of singularities: the minimal-elliptic surface singularity $N_{16}$. The class $N_{16}$ sits immediately after the simple and unimodal singularities in Arnol'd's hierarchy of singularities, and its understanding is essential to any attempt of studying deformations of singularities more complex than the unimodal ones.

The most effective tool for the study of the deformations of the unimodal singularities is the theory of deformations with $\mathbb{C}^{*}$-action of Pinkham [28. The starting point is that most of the unimodal singularities have a good $\mathbb{C}^{*}$-action such that the induced action on the tangent space to the deformations has all but one of the weights negative. The non-negative direction is topologically trivial and can be ignored. On the other hand, Pinkham's theory says that the deformations in the negative direction can be globalized and interpreted as a moduli space of certain pairs (see [19, Appendix]). Thus, the deformation problem is essentially reduced to a moduli problem for which standard algebro-geometric tools are available. For example, in the case of the triangle singularities, the pairs $(S, H)$ under consideration

Received by the editors August 28, 2006 and, in revised form, May 21, 2007.

2000 Mathematics Subject Classification. Primary 14J17, 14B07, 32S25; Secondary 14L24.

(C)2008 American Mathematical Society Reverts to public domain 28 years from publication 
consist of a $K 3$ surface $S$ and a divisor $H$ such that $H$ forms a fixed configuration of rational curves. Since the moduli space of (lattice polarized) $K 3$ surfaces is well understood, one obtains a wealth of information about the deformations of the triangle singularities (e.g. [29, 31, 5, 19]).

The case of the singularity $N_{16}$ is similar, but there is one important difference: due to the increase in modality, the zero weight directions are non-trivial and cannot be ignored. A partial modular interpretation exists for the zero weight direction as well, but in contrast to the pure negative weight situation, globalization is no longer guaranteed. As explained below, we solve this globalization problem by using GIT. Once this is done, we use the resulting moduli space to get a good hold on the structure of the deformation space of $N_{16}$. A short description of the content of the paper is given below. Further details and statements of the main results are contained in the introductions of the individual sections.

The singularity $N_{16}$ is a double cover of the cone over 5 points in $\mathbb{P}^{1}$. Consequently, following the method of Pinkham, we can essentially identify the deformations of non-positive weight of $N_{16}$ with the moduli space of pairs $(C, L)$ consisting of a plane quintic curve and a line such that the intersection is transversal. A natural approach is to construct this moduli space as a geometric invariant theory (GIT) quotient. We start by studying the moduli space of pairs $(C, L)$, where $C$ is a plane curve of degree $d$ and $L$ is a line. The moduli of such pairs is then $X / / G$, where $X \cong|d L| \times|L|$ is the parameter space for pairs and $G=\mathrm{SL}(3)$ acts naturally via the diagonal action. By definition, the construction depends on the choice of a linearization $\mathcal{L} \in \operatorname{Pic}^{G}(X)$, which is parameterized by a single rational parameter $t \in \mathbb{Q}_{+}$, the slope of $\mathcal{L}$. For each such choice, we obtain a moduli space of pairs $\mathcal{M}(t)$. This type of situation was analyzed in general circumstances by Thaddeus [37] and Dolgachev-Hu [8]. In particular, it is known that there exists only a finite number of non-isomorphic quotients $\mathcal{M}(t)$ related by explicit birational transformations.

In section 2, we establish a number of general qualitative results (valid for any degree $d$ ) on the dependence of the GIT stability for degree $d$ pairs $(C, L)$ on the parameter $t$. Namely, there are two main results here. The first result, the interpolation theorem (Theorem 2.4), says that the stability at $t=0,1$ and $\frac{d}{2}$ is equivalent to the stability of $C$ as a degree $d$ plane curve, of $C+L$ as a degree $d+1$ curve, and of the intersection $C \cap L$ as a $d$-tuple of points in $\mathbb{P}^{1}$ respectively. The second result (Theorem 2.5) relates the stability of the pair $(C, L)$ for the slope $t$ with the singularities of the divisor pair $\left(\mathbb{P}^{2}, \frac{3}{d+t}(C+t L)\right)$. Namely, if the pair $\left(\mathbb{P}^{2}, \frac{3}{d+t}(C+t L)\right)$ is $\log$ canonical, then $(C, L)$ is semistable for the slope $t$. We note that this is a relative version of earlier results of Hacking [12, $\S 10$ ] and Kim-Lee [14]. As a consequence of these two results, the dependence of the stability condition on the parameter $t$ can be roughly stated as saying that as $t$ increases from 0 to $\frac{d}{2}$ the curve $C$ is allowed to have more complicated singularities, but we require stronger transversality conditions on the intersection $C \cap L$ (see Example 2.6).

Also in section 2, we note that the variation of GIT for the pairs $(C, L)$ is closely related to the deformations of the cones over $d$-tuples of points in $\mathbb{P}^{1}$ (see (2.4). The basic observation is that, due to the interpolation theorem, the minimal closed orbits at $t=\frac{d}{2}$ are the pairs $(C, L)$ with $C$ a cone and $L$ a line not passing through the vertex of $C$. Furthermore, if $C$ is not a cone and $L$ is a transversal, the pair $(C, L)$ is stable at $t=\frac{d}{2}-\epsilon$ for $\epsilon$ small. It follows then that the variation 
of the GIT morphism $\mathcal{M}\left(\frac{d}{2}-\epsilon\right) \rightarrow \mathcal{M}\left(\frac{d}{2}\right)$ is a global object associated to the natural retraction map $S_{\leq 0} \rightarrow S_{0}$ modulo the $\mathbb{C}^{*}$-action, where $S_{\leq 0}$ and $S_{0}$ denote the deformations of the cones of non-positive and zero weights respectively. The fibers of $\mathcal{M}\left(\frac{d}{2}-\epsilon\right) \rightarrow \mathcal{M}\left(\frac{d}{2}\right)$ are (at least generically) weighted projective spaces corresponding to the negative weight deformations modulo the $\mathbb{C}^{*}$-action. In other words, the variation of GIT quotients as $t$ increases from $\frac{d}{2}-\epsilon$ to $\frac{d}{2}$ is the standard globalization of Pinkham in a relative version over the zero weight deformations (i.e. the equisingular stratum).

In section 3, we do a detailed analysis of the stability condition for the degree 5 case. The general results of section 2 specialize nicely in this situation, greatly simplifying the analysis. We restrict here to the discussion of the special role played by the slope $t=1$. First, from Theorem 2.4 it follows that a pair $(C, L)$ is (semi)stable at $t=1$ if and only if $C+L$ is (semi)stable as a plane sextic. Thus, a pair $(C, L)$ such that $C+L$ is reduced and has at worst simple singularities is stable for slope $t=1$ (cf. Shah [35]). We then note that the variation of GIT quotients at $t=1$ corresponds to a natural division of singularities into three large classes. Namely, assume for simplicity that $L$ is transversal to $C$, then the pair $(C, L)$ is semistable for some $t<1$ if and only if $C$ has at worst simple singularities. For $t=1, C$ is allowed to have simple elliptic singularities. Finally, the pairs with $C$ having worst singularities become semistable only for some $t>1$. By taking the double cover of $\mathbb{P}^{2}$ branched along $C+L$, this division of singularities into three types with respect to the stability condition is conceptually explained by Theorem 2.5] and the well-known division of the surface singularities: canonical (the rational double points), log canonical (the simple elliptic and cusp singularities), and not $\log$ canonical. For us, this division is relevant due to a theorem of Shah [34] which says that the log canonical surface singularities are insignificant limit singularities. As a consequence, we can relate the GIT construction of sections 2 and 3 to the Hodge theoretical construction of section 4.

In section 4, we note that there exists a simple alternative construction of the moduli space of degree 5 pairs. Namely, we view a degree 5 pair $(C, L)$ as a plane sextic $B=C+L$ and associate to it the double cover $S_{(C, L)}$ of $\mathbb{P}^{2}$ branched along $B$. Assuming that $(C, L)$ is a GIT stable pair at $t=1$, it follows that $S_{(C, L)}$ has only rational double points. Thus, its desingularization $\widetilde{S}_{(C, L)}$ is a degree two $K 3$ surface. The special nature of the sextic $B$ imposes conditions on the NeronSeveri lattice of $\widetilde{S}_{(C, L)}$. It follows that $\widetilde{S}_{(C, L)}$ is an $M$-polarized $K 3$ surface (see [7) for a certain rank 6 hyperbolic lattice $M$. The moduli space of $M$-polarized $K 3$ surfaces is well known to be locally symmetric of type $\mathcal{D} / \Gamma$ for appropriate choices of a type IV domain $\mathcal{D}$ and of an arithmetic group $\Gamma$ acting on $\mathcal{D}$. The main result of section 4 . Theorem 4.2. says then that this construction gives an isomorphism $\mathcal{M}(1) \cong(\mathcal{D} / \Gamma)^{*}$ between the GIT quotient $\mathcal{M}(1)$ and the Baily-Borel compactification $(\mathcal{D} / \Gamma)^{*}$ of $\mathcal{D} / \Gamma$. In conclusion, we obtain a dual description for $\mathcal{M}(1)$. As explained below, this fact has numerous consequences on the structure of the deformations of $N_{16}$.

In the last section, we discuss the implications of the results described above on the structure of deformations of the singularity $N_{16}$. Similarly to the situation of unimodal singularities, we analyze the structure of the discriminant hypersurface in the versal deformation of $N_{16}$ and the possible combinations of singularities occurring in a nearby fiber. Our main conclusion is that essentially all the results 
of Pinkham, Looijenga, and Brieskorn for unimodal singularities have a natural counterpart in our situation (see Theorems 5.6, 5.7, and 5.13).

There are two main ideas involved in the study of the deformations of $N_{16}$. First, from the results of section 2 the correct global object associated to the deformations of non-positive weight of $N_{16}$ is the fibration $\mathcal{M}\left(\frac{5}{2}-\epsilon\right) \rightarrow \mathcal{M}\left(\frac{5}{2}\right)$. However, by itself, this description does not give much. Thus, the second main idea is to gain information about the deformation space by exploiting the description of the moduli space of degree 5 pairs as the quotient $\mathcal{D} / \Gamma$. The basic observation that makes this useful is that Hodge theory transforms many questions about singularities into purely arithmetic statements. There exists, however, a disadvantage to this approach. Namely, the Hodge-theoretical arguments work well only for the singularities of finite monodromy, i.e. the simple singularities. This means that $\mathcal{D} / \Gamma$ gives a good description only for the simple singularity stratum in the deformation space of $N_{16}$. From our point of view, this is completely natural and it is easily rectified. We recall that $\mathcal{D} / \Gamma$ and the deformation space of $N_{16}$ correspond to the GIT quotients $\mathcal{M}(1)$ and $\mathcal{M}\left(\frac{5}{2}-\epsilon\right)$ respectively. Thus, the information that is missing from the $\mathcal{D} / \Gamma$ description can be recovered by following the series of explicit birational modifications that relate $\mathcal{M}(1)$ and $\mathcal{M}\left(\frac{5}{2}-\epsilon\right)$. Geometrically, the variation of GIT quotients $\mathcal{M}(1) \rightarrow \mathcal{M}\left(\frac{5}{2}-\epsilon\right)$ can be interpreted as introducing, one at a time, the non-simple singularity strata in the deformation of $N_{16}$.

One interesting aspect concerning the dual construction (GIT/Hodge theory) of this paper is that the flips that transform $\mathcal{M}(1)$ into $\mathcal{M}\left(\frac{5}{2}-\epsilon\right)$ can be interpreted also in terms of arithmetic arrangements of hyperplanes (N.B. a priori they have only a GIT meaning). As explained in $\$ 5.3$, this is closely related to Looijenga's construction [20. Essentially, the GIT approach is dual to the approach of [20, §10]. For the deformations of the triangle singularities, the two approaches coincide. For $N_{16}$ the situation is less clear, but conjecturally we should again have a coincidence.

We close by noting that the techniques developed in this paper can be applied to other classes of singularities as well. Specifically, we have in mind the threefold singularity $O_{16}$, the cone over a cubic surface. This case would be the first example of a detailed study of the deformation space for a genuine threefold singularity (not a suspension of a surface singularity). The two key ingredients of our method (the flexibility given by GIT and the explicit model obtained via the period map) have natural counterparts for $O_{16}$. Namely, the GIT analysis adapts well in higher dimensions, and the construction of section 4 can be done by using cubic fourfolds instead of $K 3$ surfaces. Details will appear elsewhere.

Notation and conventions. For the basic GIT notions and notation, we follow Mumford [26. The conventions and notation for singularities are those of Arnol'd et al. 22. The only notable difference is the use of $\widetilde{E_{r}}$ for $r=6,7,8$ to denote the simple elliptic (parabolic) singularities. In addition to the simple $\left(A_{n}, D_{n}\right.$, and $\left.E_{r}\right)$, simple elliptic $\left(\widetilde{E_{r}}\right)$, and cusp singularities $\left(T_{p, q, r}\right)$, we are concerned with the triangle (exceptional unimodal) singularities: $Z_{11}, Z_{12}, W_{12}$, and $W_{13}$ ([2, p. 247]). The singularity $N_{16}$ is a trimodal singularity with normal form:

$$
N_{16}: f_{5}(x, y)+z^{2}
$$

where $f_{5}$ is a homogeneous degree 5 polynomial. The singularity $N_{16}$ deforms only to the simple and unimodal singularities listed above. 
Since the deformation spaces of a singularity and of its suspensions can be identified, all singularities are considered up to stable equivalence (i.e. up to adding squares of new variables). In particular, depending on the context, $N_{16}$ refers to either a surface singularity or a curve singularity.

\section{VARiation of Git quotients for pairs $(C, L)$}

In this section, we construct the moduli space of pairs $(C, L)$ consisting of a plane curve of degree $d$ and a line by using geometric invariant theory (GIT). We then relate this construction to the deformations of ordinary multiplicity $d$ points.

Definition 2.1. We call a pair $(C, L)$ consisting of a plane curve $C$ of degree $d$ and a line $L \subset \mathbb{P}^{2}$ a degree d pair. Two such pairs are equivalent if they are projectively equivalent.

The natural GIT set-up for the study of the moduli of pairs is that of the group $G=\mathrm{SL}(3)$ acting diagonally on the parameter space $X$ of degree $d$ pairs, where

$$
X=\mathbb{P}\left(\mathrm{H}^{0}\left(\mathbb{P}^{2}, \mathcal{O}_{\mathbb{P}^{2}}(d)\right)\right) \times \mathbb{P}\left(\mathrm{H}^{0}\left(\mathbb{P}^{2}, \mathcal{O}_{\mathbb{P}^{2}}(1)\right)\right) \cong \mathbb{P}^{N} \times \mathbb{P}^{2}
$$

and $N=\left(\begin{array}{c}d+2 \\ 2\end{array}\right)-1$. The GIT quotient $X / / G$ depends on the choice of an ample $G$-linearized line bundle $\mathcal{L} \in \operatorname{Pic}^{G}(X)$. Namely, by definition, we have

$$
X / / \mathcal{L}_{\mathcal{L}} G=\operatorname{Proj} \bigoplus_{n \geq 0} \mathrm{H}^{0}\left(X, \mathcal{L}^{\otimes n}\right)^{G} .
$$

The dependence of the quotient on the choice of the linearization was analyzed in general circumstances by Thaddeus [37] and Dolgachev-Hu 8. In our situation, since $\operatorname{Pic}^{G}(X) \cong \operatorname{Pic}(X) \cong \mathbb{Z} \times \mathbb{Z}$, the results of [8, 37. say that the quotient $X / /{ }_{\mathcal{L}} G$ depends on a single rational parameter $t \in \mathbb{Q}_{+}$, the slope of $\mathcal{L}$, and that only finitely many non-isomorphic quotients are actually obtained.

Definition 2.2. An ample linearization $\mathcal{L} \in \operatorname{Pic}^{G}(X)$ is said to be of slope $t \in \mathbb{Q}_{+}$ if $\mathcal{L} \cong \pi_{1}^{*} \mathcal{O}_{\mathbb{P}^{N}}(a) \otimes \pi_{2}^{*} \mathcal{O}_{\mathbb{P}^{2}}(b)$ with $t=\frac{b}{a}$. We denote by $X^{s}(t)$ and $X^{s s}(t)$ the sets of stable points and semistable points respectively. The corresponding GIT quotient is denoted by $\mathcal{M}(t)$ or $X / / t G$. A point $x \in X^{s s}(t)$ will be called $t$-semistable or semistable at $t$ (and similarly for stable and unstable points). All these notions depend only on $t$.

Remark 2.3. The definitions make sense also for the two extremal cases $\mathcal{L}=$ $\pi_{1}^{*} \mathcal{O}_{\mathbb{P}^{N}}(1)$ and $\mathcal{L}=\pi_{2}^{*} \mathcal{O}_{\mathbb{P}^{2}}(1)$. We talk about linearizations of slope 0 and $\infty$. It is immediate that $\mathcal{M}(0)$ is isomorphic to the moduli space of degree $d$ plane curves and that $\mathcal{M}(\infty)=\emptyset$.

From the general results of the theory of variation of GIT quotients, it follows that there exists a finite number of critical slopes, say $0=t_{0}<t_{1}<\cdots<t_{n}<\infty$, such that:

i) $\mathcal{M}(t) \neq \emptyset$ iff $t \in\left[t_{0}, t_{n}\right]$;

ii) $\mathcal{M}(t)$ is birational to $\mathcal{M}\left(t^{\prime}\right)$ for all $t, t^{\prime} \in\left(t_{0}, t_{n}\right)$ (N.B. $\mathcal{M}\left(t_{0}\right)$ and $\mathcal{M}\left(t_{n}\right)$ are lower dimensional);

iii) $\mathcal{M}(t) \cong \mathcal{M}\left(t^{\prime}\right)$ for $t, t^{\prime} \in\left(t_{i}, t_{i+1}\right)($ for $i=0, \ldots, n-1)$; 
iv) For small $\epsilon>0$, we have the following commutative diagram:

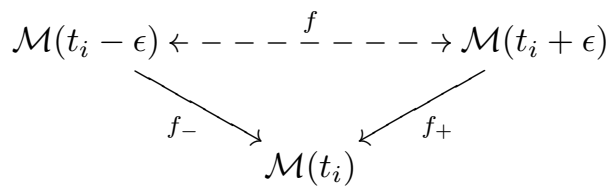

with $f_{-}, f_{+}$birational morphisms, and $f$ a flip (for $i=1, \ldots, n-1$ ). Furthermore, for the extremal critical values, there exist fibration morphisms $\mathcal{M}\left(t_{0}+\epsilon\right) \rightarrow \mathcal{M}\left(t_{0}\right)$ and $\mathcal{M}\left(t_{n}-\epsilon\right) \rightarrow \mathcal{M}\left(t_{n}\right)$.

The standard terminology is to call the critical slopes $t_{i}$ walls, the intervals $\left(t_{i}, t_{i+1}\right)$ chambers, and the birational modifications from iv) wall crossings.

In the context of these general results, we are interested in the following questions:

(Q1) Given a pair $(C, L)$, for which values of the slope $t \in \mathbb{Q}_{+}$is $(C, L)$ a semistable pair?

(Q2) Find the critical values $t_{1}, \ldots, t_{n}$ and describe their geometric relevance.

(Q3) Describe the wall crossing that occurs for slope $t_{i}$.

In this section, we establish a series of qualitative answers (valid for all degrees) to these questions. The degree 5 case is then considered in detail in section 3 ,

The first general result concerning the stability of degree $d$ pairs is to identify the boundary walls $t_{0}=0$ and $t_{n}=\frac{d}{2}$ and to describe the stability condition for the slopes 0,1 and $\frac{d}{2}$.

Theorem 2.4. Let $(C, L)$ be a degree d pair. Then, there exists an interval (possibly empty) $[\alpha, \beta] \subset\left[0, \frac{d}{2}\right]$ such that $(C, L)$ is t-semistable if and only if $t \in[\alpha, \beta]$. Furthermore,

i) $\alpha=0$ if and only if $C$ is a semistable degree d plane curve. If $C$ is stable as a plane curve, we also have $\beta>0$.

ii) $1 \in[\alpha, \beta]$ if and only if $C+L$ is semistable as a degree $d+1$ plane curve. If $C+L$ is stable as a plane curve, then $\alpha<1<\beta$.

iii) $\beta=\frac{d}{2}$ if and only if $C \cap L$ forms a semistable $d$-tuple of points in $L \cong \mathbb{P}^{1}$. If $C \cap L$ is stable as a d-tuple and $C$ is not a cone, then $\alpha<\frac{d}{2}$.

The theorem says that $t$ interpolates between two conditions of stability: the stability of degree $d$ curves in $\mathbb{P}^{2}$ (at $\left.t=0\right)$ and the stability of $d$-tuples of points in $\mathbb{P}^{1}\left(\right.$ at $\left.t=\frac{d}{2}\right)$. This can be rephrased as saying that, as $t$ increases, we allow $C$ to be more singular, but require stronger transversality conditions for $C \cap L$. More precisely, we have (see also [12, §10], [14]):

Theorem 2.5. Let $(C, L)$ be a degree d pair. If the pair $\left(\mathbb{P}^{2}, \frac{3}{d+t}(C+t L)\right)$ is log canonical, then $(C, L)$ is t-semistable.

The following example illustrates Theorems 2.4 and 2.5

Example 2.6. For degree 3 pairs, the critical slopes are $t_{0}=0, t_{1}=\frac{3}{5}, t_{2}=1$ and $t_{3}=\frac{3}{2}$. The stability of a degree 3 pair $(C, L)$ is described by the following rules:

i) If $L$ passes through a singular point of $C$, then the pair is $t$-unstable for all $t>0$. 
ii) Assume that $L$ does not meet $C$ in a singular point. Then the pair $(C, L)$ is $t$-(semi)stable iff $t \in(\alpha, \beta)(t \in[\alpha, \beta]$ respectively), where $\alpha$ and $\beta$ are given by

$\alpha=\left\{\begin{array}{ll}0 & \text { if } C \text { has at worst } A_{1} \text { singularities } \\ \frac{3}{5} & \text { if } C \text { has an } A_{2} \text { singularity } \\ 1 & \text { if } C \text { has an } A_{3} \text { singularity } \\ \frac{3}{2} & \text { if } C \text { has a } D_{4} \text { singularity }\end{array} \quad\right.$ and $\beta= \begin{cases}\frac{3}{5} & \text { if } L \text { is inflectional to } C \\ 1 & \text { if } L \text { is tangent to } C \\ \frac{3}{2} & \text { if } L \text { is transversal to } C .\end{cases}$

For degree 3 pairs, the converse of Theorem 2.5 also holds (compare with Example 2.23). For example, the case $\alpha=\frac{3}{5}$ is equivalent to saying that the log canonical threshold of a cusp is $\frac{5}{6}$.

The proofs of Theorems 2.4 and 2.5 are given in $\$ 2.2$ and 2.3 .1 respectively. Additionally, we discuss in this section the algorithmic determination of the critical slopes (2.1.2), and the relation to the theory of deformations with $\mathbb{C}^{*}$-action (\$2.4).

2.1. The numerical criterion for pairs. The main tool for investigating the dependence of the stability condition on the choice of linearization is the HilbertMumford numerical criterion ([26, Thm. 2.1]): a point $x \in X$ is stable (semistable) with respect to a linearization $\mathcal{L} \in \mathrm{Pic}^{G}(X)$ if and only if $\mu^{\mathcal{L}}(x, \lambda)>0$ (resp. $\mu^{\mathcal{L}}(x, \lambda) \geq 0$ ) for every non-trivial 1-PS $\lambda$ of $G$, where $\mu^{\mathcal{L}}(x, \lambda)$ is the numerical function of Mumford ([26, Def. 2.2]).

The functorial properties of $\mu^{\mathcal{L}}(x, \lambda)$ give the following identity:

$$
\mu^{\mathcal{O}(a, b)}(x, \lambda)=a \mu^{\mathcal{O}_{\mathbb{P}^{2}}(1)}(c, \lambda)+b \mu^{\mathcal{O}_{\mathbb{P}^{2}}(1)}(l, \lambda)=a\left(\mu(c, \lambda)+\frac{b}{a} \mu(l, \lambda)\right),
$$

where $\mathcal{O}(a, b)=\pi_{1}^{*} \mathcal{O}_{\mathbb{P}^{N}}(a) \otimes \pi_{2}^{*} \mathcal{O}_{\mathbb{P}^{2}}(b), x=(c, l) \in X \cong \mathbb{P}^{N} \times \mathbb{P}^{2}$, and $\mu(c, \lambda):=$ $\mu^{\mathcal{O}(1)}(c, \lambda)$ is the standard numerical function used for degree $d$ plane curves. Since the numerical criterion tests only for the sign of $\mu^{\mathcal{O}(a, b)}(x, \lambda)$, we can normalize by dividing by $a$. Thus, we test the (semi)stability of a point $x$ by using the function:

$$
\mu^{t}(x, \lambda):=\mu(c, \lambda)+t \mu(l, \lambda),
$$

where $t=\frac{b}{a} \in \mathbb{Q}_{+}$is the slope of the linearization $\mathcal{L}=\mathcal{O}(a, b)$. In particular, since $\mu^{t}(x, \lambda)$ is linear in $t$, we obtain the following corollary of the numerical criterion:

Corollary/Definition 2.7. For every $x \in X$ there exists a finite (possibly empty) interval $[\alpha, \beta](\alpha, \beta \in \mathbb{Q})$ such that

i) $x$ is semistable for $t \Longleftrightarrow t \in[\alpha, \beta] \cap\left(\mathbb{Q}_{+} \cup\{0\}\right)$;

ii) if $x$ is stable for some $t$, then it is stable for all $t \in(\alpha, \beta) \cap \mathbb{Q}_{+}$.

We will call the interval $[\alpha, \beta]$ the interval of stability of the point $x$.

Proof. Fix $x \in X$ and associate to a 1-PS $\lambda$ the closed interval $I_{\lambda}:=\{t \in[0, \infty) \mid$ $\left.\mu^{t}(x, \lambda) \geq 0\right\}$. By the numerical criterion, $x$ is $t$-semistable iff $t \in \bigcap_{\lambda} I_{\lambda}=:[\alpha, \beta]$. The rationality of the endpoints and the second statement follow easily from the fact that, for the application of the numerical criterion for $x$, one only needs to consider a finite number of subgroups $\lambda$ (see Remark 2.9). 
2.1.1. The numerical criterion and configurations of monomials. For applications, it is important to make $\mu^{t}(x, \lambda)$ explicit. As is customary, we choose coordinates such that the 1-PS $\lambda$ is diagonal, i.e. $\lambda$ is given by

$$
s \in \mathbb{G}_{m} \stackrel{\lambda}{\rightarrow} \operatorname{diag}\left(s^{r_{0}}, s^{r_{1}}, s^{r_{2}}\right) \in G=\mathrm{SL}(3)
$$

for some weights $r_{0}, r_{1}, r_{2} \in \mathbb{Z}$ (not all zero) with $r_{0}+r_{1}+r_{2}=0$. We assume additionally that $r_{0} \geq r_{1} \geq r_{2}$. With respect to these coordinates, a point $x \in X$ is represented by two homogenous forms $c=\sum_{i, j} c_{i j} x_{0}^{d-i-j} x_{1}^{i} x_{2}^{j}$ and $l=a_{0} x_{0}+$ $a_{1} x_{1}+a_{2} x_{2}$ of degrees $d$ and 1 respectively. Then, $\mu^{t}(x, \lambda)=\mu(c, \lambda)+t \mu(l, \lambda)$ is computed by ([26, p. 81]):

$$
\mu(c, \lambda)=\max \left\{\left(d-(i+j) r_{0}+i r_{1}+j r_{2} \mid \text { for all } i, j \text { such that } c_{i j} \neq 0\right\}\right.
$$

and

$$
\mu(l, \lambda)=\max \left\{r_{i} \mid a_{i} \neq 0\right\} .
$$

We note that the function $\mu^{t}(x, \lambda)$ depends on the slope $t \in \mathbb{Q}_{+}$and two other ingredients: the weights of $\lambda$ and the monomials occurring with non-zero coefficient in $c$ and $l$. It is convenient to further normalize $\mu^{t}(x, \lambda)$ as follows:

Definition 2.8. Let $\lambda$ be 1-PS. Fix coordinates such that $\lambda$ is diagonal with weights $r_{0}, r_{1}, r_{2}$ as above. For any $x=(c, l) \in X$, denote by $\Xi=\left(\Xi_{d}, \Xi_{1}\right)$ the set of monomials occurring with non-zero coefficient in $c$ and $l$, and call it the associated configuration of monomials. We denote $r:=\frac{r_{1}}{r_{0}}$ (the normalized weight of $\lambda$ ) and $\|\lambda\|:=r_{0}$ (the norm of $\lambda$ ). We then define

$$
\mu^{t}(\Xi, r):=\frac{\mu^{t}(x, \lambda)}{\|\lambda\|}
$$

(and similarly $\mu\left(\Xi_{i}, r\right)$ for $\left.i=1, d\right)$.

Remark 2.9. We make the following simple observations about the previous definition:

(1) As suggested by the notation, $\mu^{t}(\Xi, r)$ depends only on $\Xi, r$, and $t$. The function $\mu^{t}(\Xi, r)$ is linear in $t$, and piecewise linear in $r$.

(2) For a fixed degree, there are only finitely many possibilities for $\Xi$. Since $r_{0}+r_{1}+r_{2}=0$ and $r_{0} \geq r_{1} \geq r_{2}$ we get $r \in\left[-\frac{1}{2}, 1\right]$.

(3) Given a degree $d$ pair $(C, L)$ we say that $\Xi$ is associated to $(C, L)$ if there exists a choice of coordinates such that $\Xi$ is associated to the defining equations $(c, l)$ of the pair. The numerical criterion can be restated as: $a$ pair $(C, L)$ is t-semistable iff for any configuration $\Xi$ associated to the pair we have $\min _{r \in\left[-\frac{1}{2}, 1\right]} \mu^{t}(\Xi, r) \geq 0$.

(4) In particular, the finiteness results of the theory of variation of GIT quotients are easily obtained in our situation. For example, it follows that for a given pair $(C, L)$, one needs to apply the numerical criterion only for a finite number of 1 -parameter subgroups $\lambda_{1}, \ldots, \lambda_{k}$ (independent of $t$ ).

From (2.1) and (2.2), we note that $\mu^{t}(\Xi, r)$ depends only on the "maximal (or support) monomials" of $\Xi$. To make this precise, we introduce the following notions (see [24, Ch. 7]). 
Definition 2.10. Let $m=x_{0}^{a} x_{1}^{b} x_{2}^{c}$ be a monomial, and $r$ a rational number. We denote $\langle m, r\rangle:=a+b r-c(1+r)$ and define a partial ordering on the set of monomials of a given degree by $m>m^{\prime}$ iff $m \neq m^{\prime}$ and $\langle m, r\rangle \geq\left\langle m^{\prime}, r\right\rangle$ for all $r \in\left[-\frac{1}{2}, 1\right]$. For a set of degree $k$ monomials $\Xi_{k}$ we define the support $\operatorname{Supp}\left(\Xi_{k}\right)$ as the subset of maximal monomials with respect to this partial ordering. Similarly, for $\Xi=\left(\Xi_{d}, \Xi_{1}\right)$ we set $\operatorname{Supp}(\Xi):=\left(\operatorname{Supp}\left(\Xi_{d}\right), \operatorname{Supp}\left(\Xi_{1}\right)\right)$.

2.1.2. Algorithmic description of stability. For a fixed slope $t$, the analysis of stability for pairs is roughly equivalent to the analysis of stability for degree $d+t$ curves (see $\$ 2.2 .2$ below). This case is well known ([25, \$1.9], [24, §7.2]). Thus, we are essentially done once we have reduced the analysis to a finite number of critical values of $t$. This is achieved by the following algorithm:

Step 1 (Find the critical slopes $t_{i}$ ): For a given degree $d$, the number of configurations $\Xi$ is finite. For each $\Xi, \mu^{t}(\Xi, r)$ is continuous, piecewise linear in $r$, with critical points depending only on $\Xi$. It suffices to apply the numerical criterion only for the critical points $\left\{r_{0}=-\frac{1}{2}, \ldots, r_{k}=1\right\}$. Given $\Xi$ and a critical point $r_{i}, \mu^{t}\left(\Xi, r_{i}\right)$ is linear in $t$, changing sign at most once. Thus, the set of critical slopes is included in the set $T:=\left\{t \mid \mu^{t}\left(\Xi, r_{i}\right)=\right.$ $0, \mu^{t-\epsilon}\left(\Xi, r_{i}\right) \neq 0$ for some $\Xi$ and $\left.r_{i}\right\}$. A post-processing step removes the irrelevant slopes from $T$ (e.g. $t \in T$ with $t>\frac{d}{2}$ ). The effectivity follows from the fact that once $\Xi$ is fixed, the algorithm is linear, and the outcome depends only on $\operatorname{Supp}(\Xi)$. The number of possible supports is of order $O\left(2^{d}\right)$.

Step 2 (For each $t_{i}$ find the maximal relevant configurations): This is the standard analysis of stability for plane curves (compare [25, 1.11] with Figure 11). Essentially, one has to consider only the configurations $\Xi$ such that $\mu^{t_{i}}(\Xi, r)=0$ for some $r \in\left[-\frac{1}{2}, 1\right]$.

Step 3 (Interpret geometrically the results of Step 2): Again, this is the standard analysis of stability (e.g. [26, pp. 81-82]). The only slight difference is that we have to analyze in addition to $C$ also the relative position of $L$ (see Remark 2.12).

The first two steps are purely combinatorial, and are easily implemented. In contrast, the last step requires a careful geometric analysis, which is possible only for low degree pairs.

Remark 2.11. We can visualize the stability condition by drawing the monomials of a configuration $\Xi=\left(\Xi_{d}, \Xi_{1}\right)$ in a triangle in the plane as in Mumford [25, §1.9]. For the monomials in $\Xi_{1}$, we use the vertices of the triangle in the obvious way. The stability of $\Xi$ depends only on the support monomial of $\Xi_{1}$ and the boundary $\Gamma$ of the convex span of the support monomials of $\Xi_{d}$ (i.e. the Newton diagram). The configuration is unstable at $t=0$ (the plane curve case) iff the center of the triangle lies above the boundary $\Gamma$. More generally for slope $t$, we have the same rule, but $\Gamma$ is translated with $\frac{2 t}{3}$ units in the direction of the support monomial of $\Xi_{1}$. An example is given in Figure 1. There, $\Xi$ is $t$-semistable iff $t \geq \frac{3 D}{2}$.

Remark 2.12. For the geometric analysis of stability, an important role is played by the observation that a 1-PS $\lambda$ singles out a bad flag $p_{\lambda} \in L_{\lambda}$ ([26, p. 82]). With our convention on coordinates, we have $p_{\lambda}=(1: 0: 0)$ and $L_{\lambda} \equiv\left(x_{2}=0\right)$. A simple useful fact is that the relative position of the flag $\left(p_{\lambda}, L_{\lambda}\right)$ with respect to $L$ 


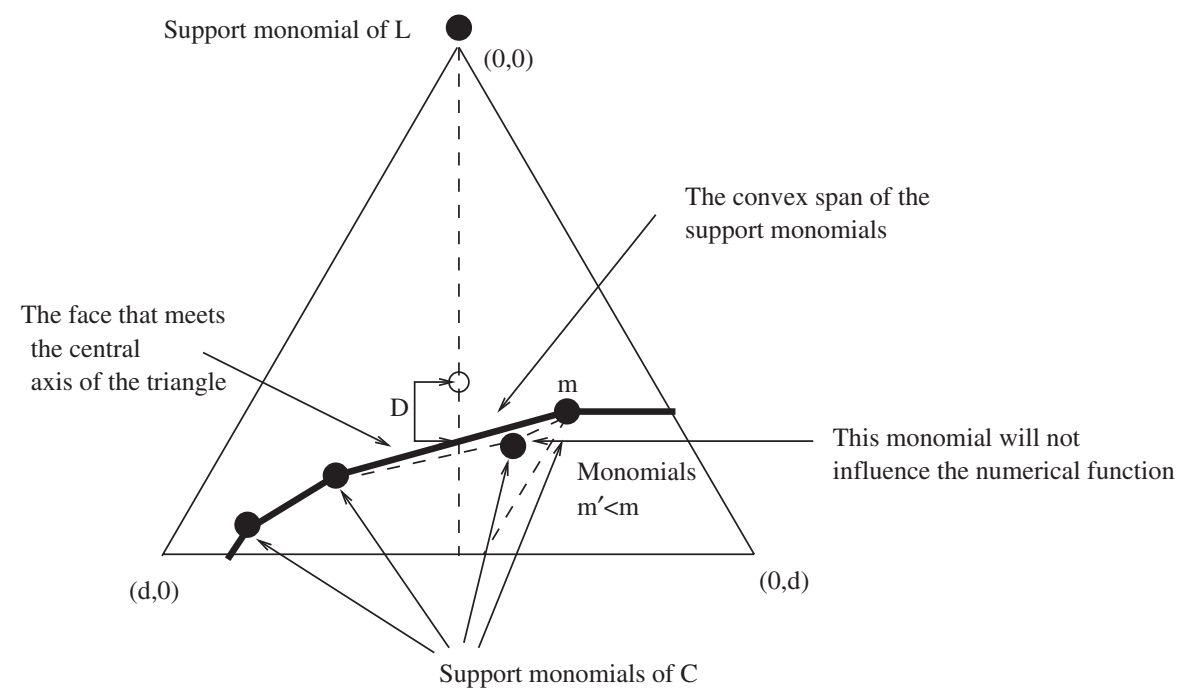

Figure 1. Configurations of monomials and stability

determines the support of $\Xi_{1}$ :

$$
\operatorname{Supp}\left(\Xi_{1}\right)= \begin{cases}x_{0} & \text { iff } p_{\lambda} \notin L, \\ x_{1} & \text { iff } p_{\lambda} \in L \text { but } L \neq \equiv L_{\lambda}, \\ x_{2} & \text { iff } L \equiv L_{\lambda} .\end{cases}
$$

2.2. Proof of Theorem 2.4. The theorem follows from Corollary 2.7 and the analysis of stability condition at the slopes 0,1 , and $\frac{d}{2}$. The notation is that of 2.1. In particular, $x=(c, l)$ is a point representing the pair $(C, L)$.

2.2.1. Slope $t=0$ case. Since $\mu^{0}(x, \lambda)=\mu(c, \lambda)$, the statement i) of Theorem 2.4 follows immediately from the numerical criterion.

Remark 2.13. The linearization of slope 0 defines the projection $X=\mathbb{P}^{N} \times \mathbb{P}^{2} \stackrel{\pi_{1}}{\longrightarrow}$ $\mathbb{P}^{N}$. Thus, $\mathcal{M}(0)$ is precisely the GIT quotient corresponding to degree $d$ plane curves. The variation of quotients morphism $\mathcal{M}(\epsilon) \stackrel{\pi}{\rightarrow} \mathcal{M}(0)$ can be interpreted as the forgetful map $(C, L) \rightarrow C$ which, over the locus of curves with trivial stabilizer, is a $\mathbb{P}^{2}$-bundle in the étale topology.

2.2.2. Slope $t=1$ case. Assume more generally that $t$ is any positive integer. We claim that $x$ is $t$-semistable iff $c \cdot l^{t}$ is semistable as a degree $d+t$ homogeneous form. This follows from the numerical criterion and the identity:

$$
\mu^{t}(x, \lambda)=\mu\left(c \cdot l^{t}, \lambda\right) .
$$

Alternatively, we can see ii) of Theorem 2.4 more intrinsically as follows. Let $V$ be the standard representation of $G=\mathrm{SL}(3)$. By definition, we have $X=$ $\mathbb{P}\left(\operatorname{Sym}^{d}\left(V^{*}\right)\right) \times \mathbb{P}\left(V^{*}\right)$. The choice of linearization $\mathcal{L}=\mathcal{O}(1, t)$ of slope $t$ gives the embedding:

$$
X=\mathbb{P}\left(\operatorname{Sym}^{d}\left(V^{*}\right)\right) \times \mathbb{P}\left(V^{*}\right) \hookrightarrow \mathbb{P}\left(\operatorname{Sym}^{d}\left(V^{*}\right) \otimes \operatorname{Sym}^{t}\left(V^{*}\right)\right)
$$


(a composition of the Veronese and Segre embeddings). By definition, the stability with respect to the linearization $\mathcal{L}$ is the stability with respect to the linear action of $G$ on $\mathbb{P}\left(\operatorname{Sym}^{d}\left(V^{*}\right) \otimes \operatorname{Sym}^{t}\left(V^{*}\right)\right)$. The representation of $G$ on $\operatorname{Sym}^{d}\left(V^{*}\right) \otimes$ $\operatorname{Sym}^{t}\left(V^{*}\right)$ is reducible with the top summand $\operatorname{Sym}^{d+t}\left(V^{*}\right)$ determined by the multiplication map:

$$
\operatorname{Sym}^{d}\left(V^{*}\right) \otimes \operatorname{Sym}^{t}\left(V^{*}\right) \stackrel{\pi}{\longrightarrow} \operatorname{Sym}^{d+t}\left(V^{*}\right) .
$$

The conclusion now follows by noting that the affine cone over the image of $X$ consists of pure tensors, none lying in the kernel of the projection $\pi$.

Remark 2.14. The morphism $X \stackrel{j}{\rightarrow} \mathbb{P}\left(\operatorname{Sym}^{d+t}\left(V^{*}\right)\right)$ constructed above is a normalization onto the image. The statement descends also to the GIT quotients.

2.2.3. Slope $t=\frac{d}{2}$ case. We note first that there are no semistable points for $t>\frac{d}{2}$.

Lemma 2.15. If $t>\frac{d}{2}$, then $X^{s s}(t)=\emptyset$. Similarly, $X^{s}\left(\frac{d}{2}\right)=\emptyset$.

Proof. Let $x=(c, l) \in X$. Choose coordinates such that $l=x_{2}$. Let $\lambda$ be the diagonal 1-PS of weights $r_{0}=r_{1}=1$ and $r_{2}=-2$. From (2.1) and (2.2), we get

$$
\mu(l, \lambda)=r_{2}=-2 \text { and } \mu(c, \lambda) \leq d,
$$

and then

i.e. $x$ is unstable.

$$
\mu^{t}(x, \lambda)=\mu(c, \lambda)+t \mu(l, \lambda)<0
$$

On the other hand, there exist $t$-semistable points for $t=\frac{d}{2}$.

Lemma 2.16. Let $(C, L)$ be a degree d pair. Assume that $L$ is transverse to $C$. Then $(C, L)$ is semistable at $t=\frac{d}{2}$.

Proof. We consider the following discriminant hypersurface in $X$ :

$$
\Sigma_{1}=\{(C, L) \mid L \text { is tangent to } C \text { or worse }\} .
$$

Clearly, $\Sigma_{1}$ defines a $G$-invariant divisor in $X$. Thus, $\Sigma_{1}$ gives an invariant section $\sigma_{1} \in H^{0}(X, \mathcal{O}(a, b))^{G}$ for some $(a, b)$. The invariant section $\sigma_{1}$ is non-vanishing exactly when $L$ is transverse to $C$. Therefore, such pairs will be semistable at $t=\frac{b}{a}$. It remains to find $(a, b)$, the bidegree of $\Sigma_{1}$. Fixing $C$ generic, we find that $b$ is the degree of the dual curve $\check{C}$, i.e. $b=d(d-1)$. Similarly, $a=2(d-1)$ is the degree of the discriminant for degree $d$ binary forms. We obtain $t=\frac{d}{2}$ and the lemma follows.

We now conclude the proof of Theorem 2.5 by the following lemma.

Lemma 2.17. A pair $(C, L)$ is semistable at $t=\frac{d}{2}$ if and only if $L$ is not a component of $C$ and $\operatorname{mult}_{p}(C \cap L) \leq \frac{d}{2}$ for every $p \in C \cap L$.

Proof. The "only if" part follows as in Lemma 2.15. Namely, given a point $p$ with $k=\operatorname{mult}_{p}(C \cap L)>\frac{d}{2}$, we choose coordinates such that $p=(1,0,0)$ and $L$ is given by $\left(x_{2}=0\right)$. It is then easy to see that there exists a choice of weights (e.g. $\left.r=\frac{k-2}{k+1}\right)$ such that the resulting diagonal 1-PS $\lambda$ destabilizes the pair $(C, L)$.

Conversely, assume that $L \not \subset C$ and $\operatorname{mult}_{p}(C \cap L) \leq \frac{d}{2}$ for all $p$. We claim that the pair is semistable at $\frac{t}{2}$. If we suppose not, then we obtain a contradiction as follows. Choose a destabilizing 1-PS $\lambda$. Normalizing as in Definition 2.8, we 
replace the pair $(C, L)$ by a configuration of monomials $\Xi=\left(\Xi_{d}, \Xi_{1}\right)$ and $\lambda$ by its normalized weight $r \in\left[-\frac{1}{2}, 1\right]$. The assumption that $\lambda$ destabilizes $(C, L)$ is equivalent to

$$
\mu^{\frac{d}{2}}(\Xi, r)=\mu\left(\Xi_{d}, r\right)+\frac{d}{2} \mu\left(\Xi_{1}, r\right)<0 .
$$

The proof now consists in analyzing the inequality (2.4) and deducing geometric consequences. Depending on the monomials occurring in $\Xi_{1}$ we distinguish 3 cases:

Case $\left(\operatorname{Supp}\left(\Xi_{1}\right)=\left\{x_{0}\right\}\right)$ : From (2.2) and the normalization procedure, we get $\mu\left(\Xi_{1}, r\right) \equiv 1$. Thus,

$$
\mu\left(\Xi_{d}, r\right)<-\frac{d}{2} \text { for some } r \in\left[-\frac{1}{2}, 1\right] .
$$

The function $\mu\left(\Xi_{d}, r\right)$ is computed by (2.1) with the weights normalized by $r_{0}=1, r_{1}=r$, and $r_{2}=-1-r$. A simple analysis gives that all the degree $d$ monomials contained in $\Xi_{d}$ must be divisible by $x_{2}^{\left\lfloor\frac{d}{2}\right\rfloor}+1$. Geometrically, this means that $C$ contains a line with multiplicity strictly greater than $\frac{d}{2}$, contradicting the assumption on the intersection $C \cap L$.

The argument in the remaining cases is similar. We obtain the following contradictions to the hypothesis:

Case $\left(\operatorname{Supp}\left(\Xi_{1}\right)=\left\{x_{1}\right\}\right): L$ passes through a point $p$ with $\operatorname{mult}_{p}(C)>\frac{d}{2}$.

Case $\left(\operatorname{Supp}\left(\Xi_{1}\right)=\left\{x_{2}\right\}\right): L$ is tangent to $C$ with multiplicity greater than $\frac{d}{2}$.

From the previous lemma and general results of the variation of GIT quotients, we obtain the following result regarding the structure of the GIT quotient at $t=\frac{d}{2}$.

Corollary 2.18. The space $\mathcal{M}\left(\frac{d}{2}\right)$ is naturally isomorphic to $\operatorname{Sym}^{d}\left(\mathbb{P}^{1}\right) / / \operatorname{SL}(2)$, the moduli of unordered d-tuples in $\mathbb{P}^{1}$. The variation of quotients morphism $\mathcal{M}\left(\frac{d}{2}-\epsilon\right) \stackrel{\pi}{\rightarrow} \mathcal{M}\left(\frac{d}{2}\right)$ can be interpreted as the forgetful map $(C, L) \rightarrow C \cap L \subset L \cong \mathbb{P}^{1}$. Furthermore, for $d \geq 5, \mathcal{M}\left(\frac{d}{2}-\epsilon\right) \rightarrow \mathcal{M}\left(\frac{d}{2}\right)$ is generically a weighted projective bundle in the étale topology.

Proof. We define a map $\operatorname{Sym}^{d}\left(\mathbb{P}^{1}\right) \rightarrow X=\mathbb{P}\left(\operatorname{Sym}^{d}\right) \times \mathbb{P}^{2}$ by associating to a $d$ tuple of points in $\mathbb{P}^{1}$ the projective cone $C$ over it together with a line $L$ not passing through the vertex. We make two basic observations:

(1) The pair $(C, L)$ is semistable at $t=\frac{d}{2}$ (cf. Lemma 2.17). The orbit of any pair $\left(C^{\prime}, L^{\prime}\right)$ with $C^{\prime} \cap L^{\prime} \cong C \cap L$ (as $d$-tuples) contains in its closure the orbit of $(C, L)$.

(2) The pair $(C, L)$ is stabilized by a 1-PS $\lambda$ of weights $(1,1,-2)$. Generically, for $d \geq 5$, the stabilizer of $(C, L)$ is precisely $\lambda$.

The first item guarantees that the induced map $\operatorname{Sym}^{d}\left(\mathbb{P}^{1}\right) / / \operatorname{SL}(2) \stackrel{j}{\rightarrow} \mathcal{M}\left(\frac{d}{2}\right)$ is well defined and surjective. From (2) and a theorem of Luna [21, Main Thm.] it follows that $j$ is actually a finite morphism. Clearly, $j$ has degree 1 . Thus, since both the source and the target of $j$ are normal varieties, the morphism $j$ is an isomorphism.

The statement concerning the structure of the variation of quotients morphism follows from Thaddeus [37, Thm. 5.6] and Dolgachev-Hu [8, Thm. 4.2.7]. 


\subsection{Relation to the log canonical threshold.}

2.3.1. Proof of Theorem 2.5. The case $t=0$ was previously established by Hacking [12, §10] and Kim-Lee [14]. The general case is essentially the same. Namely, in $\$ 2.2 .2$, we noted that the identity (2.3) implies that a pair $(C, L)$ is $t$-semistable if and only if $C+t L$ satisfies the numerical criterion for degree $d+t$ curves (N.B. the condition is purely numerical, so it makes sense for $t \in \mathbb{Q})$. Assume that $(C, L)$ is $t$-unstable. By the numerical criterion, we can find coordinates $\left(x_{0}: x_{1}: x_{2}\right)$ on $\mathbb{P}^{2}$ and relatively prime integral weights $w_{1}, w_{2}$ such that with respect to the affine coordinates $x=\frac{x_{1}}{x_{0}}$ and $y=\frac{x_{2}}{x_{0}}$ at $p=(1: 0: 0)$ we have

$$
w(f)>\frac{d+t}{3}\left(w_{1}+w_{2}\right)
$$

where $f(x, y)$ is the equation of $C+t L$ and $w(f)$ denotes the order of $f$ at $p$. By considering the weighted blow-up of $\mathbb{P}^{2}$ with respect to the weights $w_{1}$, $w_{2}$, we obtain via a standard discrepancy computation (see [17, 6.38]) that

$$
a\left(E, \mathbb{P}^{2}, \frac{3}{d+t}(C+t L)\right)=\left(w_{1}+w_{2}-1-\frac{3}{d+t} \cdot w(f)\right)<-1,
$$

where $E$ is the exceptional divisor of the blow-up and $a(\ldots)$ denotes the discrepancy of $E$ (see [16, §2.3]). We conclude that $\left(\mathbb{P}^{2}, C+t L\right)$ is not log canonical.

2.3.2. The stability threshold. Let $(C, L)$ be a degree $d$ pair and $[\alpha, \beta] \subset\left[0, \frac{d}{2}\right]$ its interval of stability. As a consequence of Theorem 2.5. it follows that the endpoints $\alpha$ and $\beta$ of the interval of stability are essentially determined by the worst singularity of $C$ and by the point of highest multiplicity for the intersection $C \cap L$ respectively. To simplify the computations involved in the complete analysis of stability for a given degree $d$ (e.g. $d=5$ in section 3), it is convenient to make the statement of Theorem 2.5 more explicit. Specifically, we measure the effect of the singularities of $C$ on the stability of the pair $(C, L)$ by the following notion:

Definition 2.19. Let $C$ be a plane curve of degree $d$ and $p \in C$ a singular point. We define the stability threshold of $p \in C$, denoted by $t_{p}(C)$, to be

$$
t_{p}(C):=-\inf _{\lambda \text { adapted to } p} \frac{\mu(c, \lambda)}{\|\lambda\|},
$$

where a 1-PS $\lambda$ is adapted to $p$ iff $p=p_{\lambda}$, the norm of $\lambda$ is $\|\lambda\|:=r_{0}$ (the highest weight of $\lambda$ ), and $c$ is the equation of $C$.

Note that the stability threshold does not depend on $L$. We then have:

Lemma 2.20. Let $(C, L)$ be a degree d pair with interval of stability $[\alpha, \beta]$. Then

$$
\alpha \leq \max \left\{\max _{p \in \operatorname{Sing}(C)} t_{p}(C), 0\right\}
$$

with equality if $L$ is transverse to $C$.

The stability threshold of a singular point is related to a well-known invariant of the singularity, the log canonical threshold (see [15] and [17, §6.5]).

Definition 2.21. Let $p \in C$ be a singular point. We say that the singularity at $p$ is linearly semi-quasihomogeneous if there exists a choice of homogeneous coordinates $\left(x_{0}: x_{1}: x_{2}\right)$ and a choice of weights $w_{1}$ and $w_{2}$ such that the associated affine 
equation $f(x, y)$ of $C$ at $p$ is semi-quasihomogeneous (i.e. the leading term $f_{w}$ defines an isolated singularity at the origin).

Corollary 2.22. The following inequality holds:

$$
t_{p}(C) \leq \frac{3}{\operatorname{lct}_{p}(C)}-d,
$$

where $\operatorname{lct}_{p}(C)$ denotes the $\log$ canonical threshold of $p \in C$. The equality holds if the singularity at $p$ is linearly semi-quasihomogeneous.

Proof. The claim follows by the same argument as in Theorem 2.5. If $p$ is a semiquasihomogeneous singularity, the log canonical threshold is computed by $\operatorname{lct}_{p}(C)=$ $\frac{w_{1}+w_{2}}{w(f)}\left(\left[15\right.\right.$, Prop. 8.14]). A similar formula holds for $t_{p}(C)$ under the assumption of linearity.

For low degree pairs, the equality in (2.7) almost always holds. We note, however, that starting with degree 4 there are examples in which this fails:

Example 2.23 (The stability threshold is not a local analytic invariant). Consider the following singularities of plane quartics: $C_{1}:\left(x^{2}+x y^{3}=0\right)$ and $C_{2}:\left(\left(x-y^{2}\right)^{2}-x^{2} y^{2}=0\right)$. Analytically, they both define an $A_{5}$ singularity at the origin $p$. Thus, the $\log$ canonical threshold $\mathrm{i}$ both cases is $\frac{2}{3}$. It is easy to compute that $t_{p}\left(C_{1}\right)=\frac{1}{2}$ and respectively $t_{p}\left(C_{2}\right)=0$. It follows that the equality in (2.7) holds only in the first case. The two situations are not distinguished locally, but globally. Namely, $C_{1}$ consists of a line tangent with multiplicity 3 to a cubic, and $C_{2}$ is the union of two conics tangent with multiplicity 3 at the point $p$.

The following bounds for the stability threshold in terms of the multiplicity of the singularity are easily established.

Proposition 2.24. Assume that $p$ is a point of multiplicity $k$ for a degree $d$ curve $C$. Then the following bounds hold:

$$
\frac{3 k}{2}-d \leq t_{p}(C) \leq 3 k-d .
$$

Moreover

i) $t_{p}(C)=\frac{3 k}{2}-d$ iff every line in the tangent cone at $p$ has multiplicity at most $\frac{k}{2}$

ii) $t_{p}(C) \leq 3 k \cdot \frac{d-1}{d+k-2}-d$ if $p$ is an isolated singularity;

iii) $t_{p}(C)=3 k-d$ iff $C$ contains a line with multiplicity $k$ passing through $p$.

Remark 2.25 . We close by noting that the stability threshold is easily computed in practice. Namely, let $p$ be an isolated singular point of multiplicity $k$ for $C$. By looking at the tangent cone, we have that either every tangent line occurs with multiplicity at most $\frac{k}{2}$ in the tangent cone, or there exists a unique special tangent $L_{0}$ with multiplicity greater than $\frac{k}{2}$. The first case is covered by the previous proposition. In the second case, it is easily seen that the 1-PS $\lambda$ that computes the stability threshold $t_{p}(C)$ has the property that $p_{\lambda}=p$ and $L_{\lambda}=L_{0}$. By choosing coordinates such that $p=(1: 0: 0)$ and the special tangent $L_{0}$ is given by $\left(x_{2}=0\right)$, we obtain a configuration $\Xi=\left(\Xi_{d}, \Xi_{1}\right)$ of monomials. The stability threshold is 
then given by $t_{p}(C)=-\min _{r \in\left[-\frac{1}{2}, 1\right]} \mu\left(\Xi_{d}, r\right)$, which is easily computed (Remark 2.11). The essential observation here is that, while $\Xi$ depends on the choice of coordinates, its support $\operatorname{Supp}(\Xi)$ does not.

2.3.3. Upper bounds for the interval of stability. We can apply similar considerations for the analysis of the failure of stability due to a point $p \in C \cap L$. Note that the singularity at $p$ of the $\mathbb{Q}$-divisor $C+t L$ becomes worse as $t$ increases. We obtain the following bounds for the endpoint $\beta$ of the interval of stability.

Proposition 2.26. Let $p$ be the point with the highest multiplicity in the intersection $C \cap L$. Then, the upper bound $\beta$ of the interval of stability of the pair $(C, L)$ satisfies the following estimates:

i) if $L$ is a component of $C$, then $\beta \leq \frac{d-3}{2}$;

ii) if $L$ is not a component of $C$ and $\operatorname{mult}_{p}(C \cap L) \leq \frac{d}{2}$, then $\beta=\frac{d}{2}$;

iii) if $L$ is not a component of $C$ and $\operatorname{mult}_{p}(C \cap L)=k>\frac{d}{2}$, then

$$
\frac{d}{2}-\frac{3}{2}(2 k-d) \leq \beta \leq \frac{d}{2}-\frac{3(2 k-d)}{2(2 k-1)} .
$$

Furthermore, the above estimates are sharp.

2.4. Relation to the deformations of non-positive weight. The motivation for the study of the moduli space of pairs comes from Pinkham's theory of deformations of singularities with $\mathbb{C}^{*}$-action. The basic idea of this theory is that in the presence of a $\mathbb{C}^{*}$-action a certain subspace of the deformation space, the deformations of negative weight, can be globalized and interpreted as a moduli space of pairs. This modular interpretation gives effective tools for the study of the deformations of certain classes of singularities, such as the unimodal singularities (e.g. [30, 29, 18, 19, 5]). For the general theory, we refer to the work of Pinkham [28, 32] (for a short exposition, see [19, Appendix]). Here, we briefly recall the basic concepts of the theory, and explain the relation between the deformations of cones over $d$-tuples of points in $\mathbb{P}^{1}$ and the variation of GIT quotients for degree $d$ pairs.

Let $\left(Y_{0}, y_{0}\right)$ be the germ of a singularity with good $\mathbb{C}^{*}$-action in the sense of Pinkham (e.g. an isolated quasihomogeneous hypersurface singularity). Then there exists a formal versal deformation $\mathcal{Y} \rightarrow S$ such that $\mathbb{C}^{*}$ acts equivariantly on $\mathcal{Y}$ and $S$. This determines subspaces $S_{-}, S_{0}, S_{\leq 0}$ and $S_{+}$and pull-back families (e.g. $\mathcal{Y}_{-} \rightarrow S_{-}$, the deformations of negative weight). Each of these spaces has an intrinsic interpretation (see 32]). In particular, the zero weight deformations are those preserving the $\mathbb{C}^{*}$-action, and the deformations of non-positive weight are the deformations that can be lifted to projective deformations of a completion $\bar{Y}_{0}$ of the singularity. Specifically, we can assume $Y_{0}=\operatorname{Spec} A$ for a graded $\operatorname{ring} A$. Then $\bar{Y}_{0}=\operatorname{Proj} A[t]$ (for $\operatorname{deg}(t)=1$ ) is a natural compactification of $Y_{0}$. The divisor $Y_{\infty} \cong \bar{Y}_{0} \backslash Y_{0}$ defined by $t$ is called the divisor at infinity. This construction works well in families and identifies the deformations of non-positive weight to the projective deformations of the pair $\left(\bar{Y}_{0}, Y_{\infty}\right)$ ([32, Thm. 2.9]). The deformations of negative weight correspond to the deformations of $\left(\bar{Y}_{0}, Y_{\infty}\right)$, fixing the hyperplane at infinity $Y_{\infty}$.

The key observation of Pinkham is that, due to the $\mathbb{C}^{*}$-action, the deformations of negative weight can be globalized in the following sense. The functor of deformations of negative weight is representable by an affine space $S_{-}$. The resulting weighted projective space $S_{-} / / \mathbb{C}^{*}$ is then the moduli of pairs $\left(\bar{Y}, Y_{\infty}\right)$, where $\bar{Y}$ 
is a projective deformation of $\bar{Y}_{0}$ and $Y_{\infty}$ is the fixed hyperplane section (for a functorial formulation, see [19, Appendix]). The globalization does not hold in the zero weight direction, but we can interpret $S_{0}$ as corresponding to the deformations of the hyperplane at infinity $Y_{\infty}$. Also, there exists a natural $\mathbb{C}^{*}$-equivariant map $S_{\leq 0} \rightarrow S_{0}$, whose fibers correspond to the deformations of negative weight.

The construction explained in the previous paragraphs is easily understood in the case of deformations of quasihomogeneous hypersurface singularities. For instance, in our situation, $Y_{0}$ is the affine cone over a $d$-tuple of points in $\mathbb{P}^{1}$ and $\bar{Y}_{0}=: C_{0}$ is the natural projective cone. The hyperplane at infinity is simply a transversal line $L$ to $C_{0}$. It follows that the deformations of negative weight of the cone $Y_{0}$ correspond to the pairs $(C, L)$ with $C \cap L$ projectively equivalent to $C_{0} \cap L$. Similarly, the nonpositive weight deformations correspond to the pairs $(C, L)$ such that $C \cap L$ is a small deformation of $C_{0} \cap L$.

Since a degree $d$ pair $(C, L)$ such that $C \cap L$ is a transversal and $C$ is not a cone is GIT stable at $t=\frac{d}{2}-\epsilon$ (cf. Theorem 2.4), we can interpret $\mathcal{M}\left(\frac{d}{2}-\epsilon\right)$ as a global object associated to the non-positive weight deformations $S_{\leq 0}$. Furthermore, the variation of the GIT map $\mathcal{M}\left(\frac{d}{2}-\epsilon\right) \rightarrow \mathcal{M}\left(\frac{d}{2}\right)$ can be interpreted as a globalization of the map $S_{\leq 0} \rightarrow S_{0}$ modulo the $\mathbb{C}^{*}$-action. To be more precise, let us recall that a point $x_{0} \in \mathcal{M}\left(\frac{d}{2}\right)$ corresponds to the closed orbit of a pair $\left(C_{0}, L\right)$ consisting of a degree $d$ cone and a transversal line. The stabilizer $G_{x_{0}}$ of the pair $\left(C_{0}, L\right)$ is $\mathbb{C}^{*}$ (at least generically, for $d \geq 5)$. Let $S_{\leq 0}$ be a normal slice to the orbit of $\left(C_{0}, L\right)$ and $S_{0}$ the invariant part. By Luna's slice theorem, locally at $x_{0}$ in the étale topology, we have the following commutative diagram:

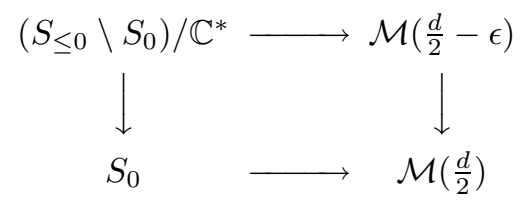

with the horizontal maps being étale. The stabilizer $G_{x_{0}} \cong \mathbb{C}^{*}$ acts naturally on the tangent space $T_{S_{\leq 0}}$. It is then a simple (almost tautological) computation to identify $T_{S_{\leq 0}}$ with the non-positive weight subspace of $\operatorname{Ext}^{1}\left(\Omega_{Y_{0}}, \mathcal{O}_{Y_{0}}\right) \cong \mathbb{C}\{x, y\} /\left\langle J\left(f_{d}\right)\right\rangle$, where $f_{d}(x, y)$ is the equation of $Y_{0}$ and $J\left(f_{d}\right)$ is the Jacobian ideal. It follows that the germ of $S_{\leq 0}$ at the origin is indeed the space of deformations of nonpositive weight, and similarly for $S_{0}$. In conclusion, (2.8) corresponds indeed to a globalization of the natural map of deformations $S_{\leq 0} \rightarrow S_{0}$. Moreover, the weighted projective fibers of $\mathcal{M}\left(\frac{d}{2}-\epsilon\right) \rightarrow \mathcal{M}\left(\frac{d}{2}\right)$ (cf. Corollary 2.18) correspond to the negative weight deformations $S_{-}$modulo $\mathbb{C}^{*}$.

Remark 2.27. The singularity $N_{16}$ is quasi-homogeneous with Milnor (and Tyurina) number $\mu=16$. The weights for $N_{16}$ are as follows: one is positive, two are zero, and 13 are negative. Since the only positive weight is in the Hessian direction, the positive weight deformations are topologically trivial and, as is customary, we ignore them.

\section{The Stability CONDitions For DEgRee 5 PAirs}

In this section, we do a detailed analysis of the stability conditions for degree 5 pairs. The first step of this analysis is the determination of the relevant critical slopes based on the algorithm described in $\$ 2.1 .2$ 
Lemma 3.1. The critical slopes for degree 5 pairs are: $0, \frac{1}{7}, \frac{1}{4}, \frac{2}{5}, \frac{5}{8}, 1, \frac{10}{7}, \frac{8}{5}, \frac{5}{3}$, $\frac{7}{4}, \frac{13}{7}, 2, \frac{11}{5}$, and $\frac{5}{2}$.

The semi-stability of the pair $(C, L)$ for the slopes 0,1 , and $\frac{5}{2}$ is equivalent to the semi-stability of $C, C+L$, and $C \cap L$ respectively (Theorem 2.4). Thus, the stability condition for the slopes 0,1 , and $\frac{5}{2}$ is well understood. In particular, as a corollary of the results of Shah on the stability of plane sextics ([35, Thm. 2.3]), we obtain:

Corollary 3.2. Let $(C, L)$ be a degree 5 pair. Assume that the sextic $C+L$ is reduced. Then the pair $(C, L)$ is stable (semistable) at $t=1$ if and only if $C+L$ has at worst simple (resp. simple elliptic or cusp) singularities.

The description of the stability for the remaining critical slopes is a standard GIT computation. For degree 5 pairs, the computation is simplified by the fact that the interval of stability can be determined by considering independently the worst singularity of $C$ and of the intersection $C \cap L$. It follows that the stability conditions are essentially determined by the results of 2.3 .2 and 2.3 .3 . The following statement summarizes the results of this section.

Theorem 3.3. Let $(C, L)$ be a degree 5 pair. The interval of stability $[\alpha, \beta] \subseteq\left[0, \frac{5}{2}\right]$ is determined by the following rules:

i) Assume that $L$ does not pass through a singularity that makes $C$ unstable. Then $\alpha$ is determined by the worst singularity of $C$, i.e.

$$
\alpha=\max _{p \in \operatorname{Sing}(C)}\left\{t_{p}(C), 0\right\} .
$$

Similarly, $\beta$ is determined by the worst intersection point of $C$ and $L$ (see $\$ 3.1$ and $\$ 3.2$ respectively).

ii) Assume that $L$ passes through a singularity that makes $C$ unstable. Then the interval of stability of the pair is either empty or consists of a single point $t=1$ (see Lemma 3.14).

In particular, it follows that the change of the stability condition is very simple for all critical slopes except $t=1$. Essentially, for such a slope, exactly one class of singularities becomes stable, and one type of degenerate intersection becomes unstable (see 33.3). The change of stability at $t=1$ is more involved (see 3.4), but it follows a pattern as noted by the following corollary:

Corollary 3.4. Let $(C, L)$ be a degree 5 pair. Assume that the intersection $C \cap L$ is a transversal and that $C$ is not a cone. Then the interval of stability of the pair $(C, L)$ is $\left[\alpha, \frac{5}{2}\right]$ with:

i) $\alpha \in[0,1)$ iff the quintic $C$ has at worst simple singularities;

ii) $\alpha=1$ iff the quintic $C$ has a simple elliptic or cusp singularity;

iii) $\alpha \in\left(1, \frac{5}{2}\right)$ iff the quintic $C$ has a triangle singularity.

The division of singularities in three large classes as above has significance both topologically (in terms of monodromy) and algebraically (i.e. canonical, log canonical, or worse singularities; see Remark 3.25). The corollary says that the division makes sense also in terms of GIT stability. The coincidence of these three different points of view plays a key role in the second part of our paper (see also the discussion from Mumford [25, §3]). 
3.1. The singularities of plane quintics and stability. The effect of a singular point $p \in C$ on the stability of a pair $(C, L)$ is measured by the stability threshold $t_{p}(C)$ as described in $\$ 2.3 .2$. Here we are interested in the possible values for $t_{p}(C)$ in the case of plane quintics.

3.1.1. Isolated singularities case. We start by noting the following list of possible singularities for a plane quintic.

Proposition 3.5 (Wall [40]). Let $C$ be a reduced plane quintic, and $p \in C$ a singular point. Then the singularity at $p$ is one of the following types:

- a simple singularity $A_{k}(k \leq 12), D_{l}(l \leq 12)$, or $E_{m}(m=6,7,8)$;

- a simple elliptic singularity $\widetilde{E}_{r}(r=7,8)$;

- a plane cusp singularity $T_{2,3, k}(k=7, \ldots, 10)$ or $T_{2, q, r}(4 \leq q \leq r \leq 6)$;

- a triangle singularity of type $Z_{11}, Z_{12}, W_{12}$, or $W_{13}$;

- a trimodal singularity of type $N_{16}$.

The simplest singularities of the list give stable plane quintics (see [26, p. 80]), and thus the corresponding stability threshold is less than 0 (Theorem 2.4).

Lemma 3.6. A plane quintic with only singularities of types $A_{k}, D_{4}$, or $D_{5}$ is GIT stable.

For the analysis of the remaining singularities, we need to separate the case of a singularity of analytic type $D_{8}$ into two subcases: either of type $D_{8}^{\prime}$ or not.

Definition 3.7. Let $p \in C$ be a singular point of a plane quintic. We say that $p$ has type $D_{8}^{\prime}$ iff the quintic $C$ decomposes as a line plus a nodal quartic such that the line is tangent with multiplicity 4 to the quartic and $p$ is both the node of the quartic and the intersection point of the two components.

For many classes of singularities of plane quintics, the stability threshold can be computed directly in terms of the log canonical threshold.

Lemma 3.8. Assume that $p \in C$ is an isolated singularity of one of the following types: $D_{6}, D_{8}^{\prime}, E_{k}(k=6,7,8), \widetilde{E_{7}}, \widetilde{E_{8}}, Z_{11}, Z_{12}, W_{12}$ or $W_{13}$. Then the singularity at $p$ is linearly semi-quasihomogeneous. In particular, the stability threshold is computed from the log canonical threshold by the formula $t_{p}(C)=\frac{3}{\operatorname{lct}_{p}(C)}-5$.

Proof. The first claim is a case-by-case analysis of the analytic type of the singularity at $p$ based on the Newton diagram. The second part follows from Corollary 2.22 .

The remaining cases are handled by the following two lemmas.

Lemma 3.9. Assume that $p \in C$ is a singularity of type $T_{2 q r}$ (with $4 \leq q \leq r \leq 6$ ) for the quintic $C$. Then we have $t_{p}(C)=1$.

Proof. The singularities of type $T_{2 q r}$ with $4 \leq q \leq r \leq 6$ have multiplicity 4 , but no line in the tangent cone has multiplicity greater than 2 . The conclusion follows from Proposition 2.24

Lemma 3.10. Let $p$ be an isolated singular point of the quintic curve $C$.

i) If $p$ is a singular point of type $D_{k}(k \geq 7)$, but not of type $D_{8}^{\prime}, t_{p}(C)=0$.

ii) If $p$ is a singular point of type $T_{23 k}(k \geq 7), t_{p}(C)=1$. 
Proof. The statement follows easily from the fact that we can choose affine coordinates at $p$ such that the leading terms (w.r.t. appropriate weights) of the defining equation of $C$ are $f_{w}(x, y)=x y^{2}+2 x^{3} y+x^{5}$ and $f_{w}(x, y)=y^{3}+2 y^{2} x^{2}+x^{4} y$ respectively (see Remark 2.25).

In conclusion, we obtain:

Proposition 3.11. Let $p \in C$ be an isolated singular point of a plane quintic. The stability threshold of $p \in C$ is computed by

$$
t_{p}(C)= \begin{cases}\leq 0 & \text { if } p \text { is of type } A_{k}, \text { or } D_{l}, \text { but not of type } D_{8}^{\prime} \\ \frac{1}{7}, \frac{1}{4}, \frac{2}{5}, \frac{5}{8} & \text { if } p \text { is of type } E_{6}, D_{8}^{\prime}, E_{7}, \text { or } E_{8} \text { respectively } \\ 1 & \text { if } p \text { is of type } \widetilde{E}_{r} \text { or } T_{2, p, q} \\ \frac{10}{7}, \frac{8}{5}, \frac{5}{3}, \frac{13}{7} & \text { if } p \text { is of type } Z_{11}, Z_{12}, W_{12}, \text { or } W_{13} \text { respectively } \\ \frac{5}{2} & \text { if } p \text { is of type } N_{16} .\end{cases}
$$

In particular, $t_{p}(C)<1$ iff $p$ is a simple singularity, $t_{p}(C)=1$ iff $p$ is a simple elliptic or cusp singularity, and $t_{p}(C)>1$ otherwise.

3.1.2. The stability conditions for non-reduced quintics. The stability of pairs $(C, L)$ such that $C$ is a non-reduced quintic is determined by the following two lemmas.

Lemma 3.12. Let $(C, L)$ be a degree 5 pair. If $C$ contains a triple line, then the pair $(C, L)$ is unstable for all slopes $t$.

If $C$ is non-reduced and contains no line with multiplicity 3 , we can write $C=$ $2 D+R$, where $D$ is either a line or a smooth conic and $R$ is the residual curve having no common component with $D$. We then have:

Lemma 3.13. Let $C=2 D+R$ be a non-reduced quintic containing no triple line. Then $\alpha=\max _{p \in \operatorname{Sing}(C)} t_{p}(C)$ is given by Table 1 .

TABle 1 . The stability of pairs $(C, L)$ with $C=2 D+R$

\begin{tabular}{|l|l|c|}
\hline The double component $D$ & Geometry of $D \cap R$ & $\alpha$ \\
\hline \hline$D$ is a smooth conic & $R$ is a secant to $D$ & 0 \\
\hline$D$ is a smooth conic & $R$ is tangent to $D$ & 1 \\
\hline \hline$D$ is a line & $|D \cap R| \geq 2$ & 1 \\
\hline$D$ is a line & $D \cap R=\{p\}$ and $p \in R$ is smooth & $\frac{7}{4}$ \\
\hline$D$ is a line & $D \cap R=\{p\}$ and $p \in R$ is of type $A_{1}$ & 2 \\
\hline$D$ is a line & $D \cap R=\{p\}$ and $p \in R$ is of type $A_{2}$ & $\frac{11}{5}$ \\
\hline$D$ is a line & $D \cap R=\{p\}$ and $p$ is a triple point of $R$ & $\frac{5}{2}$ \\
\hline
\end{tabular}

3.2. The stability for non-generic intersections. As a consequence of the relation between the GIT stability and log canonicity (see Theorem 2.5), the interval of stability of a pair $(C, L)$ is determined by the worst singularity $p$ of $C$ and the point $p^{\prime}$ of maximal multiplicity in the intersection $C \cap L$. As long as $p \neq p^{\prime}$, the contributions of the singularities of $C$ and of the intersection $C \cap L$ to the interval of 
stability are easily quantified. The situation $p=p^{\prime}$ (i.e. the line $L$ passes through a bad singularity of $C$ ) is geometrically more subtle, but in the case of quintics it is easily handled by the following result:

Lemma 3.14. Let $(C, L)$ be a degree 5 pair with interval of stability $[\alpha, \beta]$. Then,

$$
\alpha=\max _{p \in \operatorname{Sing}(C)}\left\{t_{p}(C), 0\right\}
$$

except when there exists a point $p \in C \cap L$ that makes $C$ unstable, in which case either:

i) $\alpha=1$ if $C$ is the union of a quartic $C^{\prime}$ and a 4-fold tangent line $L^{\prime}$ through a double point of $C^{\prime}$ with $L \neq L^{\prime}$ and $p=C^{\prime} \cap L^{\prime}$;

ii) $[\alpha, \beta]=\emptyset$ otherwise.

If $L$ does not pass through a singular point making $C$ unstable, then $\beta$ can be determined by considering only 1-PS $\lambda$ such that $p_{\lambda}$ is the point with the highest multiplicity in $C \cap L$.

Proof. Assume that $\alpha>\max _{p \in \operatorname{Sing}(C)}\left\{t_{p}(C), 0\right\}$. From the definition of the interval of stability, it follows that there exists a 1-PS $\lambda$ such that $\mu^{t}(x, \lambda) \geq 0$ iff $t \geq \alpha$, where $x=(c, l) \in X$ represents the pair $(C, L)$. Since $\alpha>0$ and $\mu^{t}(x, \lambda)=$ $\mu(c, \lambda)+t \mu(l, \lambda)$, we get $\mu(c, \lambda)<0$, i.e. $p:=p_{\lambda}$ is a singular point that destabilizes $C$. Since $\alpha>t_{p}(C)$, we also get $\mu(l, \lambda)<\|\lambda\|$, i.e. $p \in L$. Thus $L$ passes through a singularity which destabilizes $C$. By a computer-aided analysis, it follows that the only case when the interval of stability is non-empty is described by the lemma. The computation for $\beta$ is similar.

The following three lemmas determine the endpoint $\beta$ in terms of the geometry of the intersection $C \cap L$. We note the following two facts that simplify the computation:

(1) We can assume that either $L$ is a component of $C$, or there exists a (unique) point $p$ with $\operatorname{mult}_{p}(C) \geq 3$. Otherwise, from Theorem 2.4 it follows that $\beta=\frac{5}{2}$.

(2) We can assume that $L$ passes only through mild singularities of $C$. Otherwise, we are in the situation covered by the previous lemma.

The pairs which have $\beta>1$ play an important role in the following sections.

Lemma 3.15. Let $(C, L)$ be a degree 5 pair with interval of stability $[\alpha, \beta]$. Then, $\beta>1$ if and only if $L$ is not a component of $C$ and any point $p$ with $\operatorname{mult}_{p}(C \cap L) \geq 3$ defines a simple singularity for the total curve $C+L$. The geometric possibilities for $p \in C \cap L$ to define a simple singularity for $C+L$ and the corresponding value of $\beta$ are given in Table 2 ,

The cases where $L$ is a component of $C$ are described by the following lemma.

Lemma 3.16. Let $(C, L)$ be a degree 5 pair and let $[\alpha, \beta]$ be its interval of stability. Assume that $L$ is a component of $C$, and let $R$ be the residual curve. Then

i) If $L$ is a component of $R$, then the pair $(C, L)$ is unstable for all $t$.

ii) If $L$ is not a component of $R$, then we are in one of the following situations:

- if $\operatorname{mult}_{p}(L \cap R) \leq 2$ for all $p \in R \cap L$, then $\beta=1$;

- if $L$ passes through a singular point $p$ of $R$ and $\operatorname{mult}_{p}(L \cap R) \geq 3$, then $\beta \leq 0$; 
TABle 2. The case $p \in(C+L)$ defines a simple singularity

\begin{tabular}{|c|c|c|c|}
\hline mult $_{p}(C \cap L)$ & Sing. at $p \in C$ & Sing. at $p \in(C+L)$ & $\beta$ \\
\hline \hline$k \in\{1, \ldots, 5\}$ & smooth & $A_{2 k-1}$ & $\frac{5}{2}, \frac{5}{2}, \frac{11}{5}, \frac{13}{7}$, and $\frac{5}{3}$ resp. \\
\hline$k \in\{2, \ldots, 5\}$ & $A_{1}$ & $D_{2 k}$ & $\frac{5}{2}, 2, \frac{8}{5}$, and $\frac{10}{7}$ resp. \\
\hline 3 & $A_{2}$ & $E_{7}$ & $\frac{7}{4}$ \\
\hline 2 & $A_{n}$ & $D_{n+3}$ & $\frac{5}{2}$ \\
\hline
\end{tabular}

- if $L$ is 4-fold tangent to $R$ at a smooth point, then $\beta=\frac{1}{7}$;

- if $L$ is 3 -fold tangent to $R$ at a smooth point, then $\beta=\frac{2}{5}$.

The remaining cases are covered by the following lemma.

Lemma 3.17. Let $(C, L)$ be a degree 5 pair with interval of stability $[\alpha, \beta]$. Assume that $L$ is not a component of $C$ and that there exists a point p with mult $_{p}(C \cap L) \geq 3$. Assume additionally that the singularity at $p$ does not destabilize $C$, and that $p$ is a non-simple singularity for $C+L$. Then $\beta=1$ with the exception of the cases listed in Table 3 .

TABLE 3. Intersections that destabilize $(C, L)$ before slope $t=1$

\begin{tabular}{|c|c|c|}
\hline Sing. of $p \in C$ & Position of $L$ through $p$ & $\beta$ \\
\hline \hline$A_{7}$ & $C=L+R, L$ is 4-fold tangent to the residual quartic $R$ & $\frac{1}{7}$ \\
\hline$D_{5}$ & $L$ is a special tangent through $p$ & $\frac{1}{4}$ \\
\hline$A_{5}$ & $C=L+R, L$ is 3 -fold tangent to the residual quartic $R$ & $\frac{2}{5}$ \\
\hline$A_{4}$ & $L$ is 5 -fold tangent through $p$ & $\frac{5}{8}$ \\
\hline
\end{tabular}

We close the discussion of the GIT analysis by noting that, while the results for degree 5 generally follow some predictable patterns, there are some pathological examples. For example, there exist pairs which are strictly semistable for an entire interval (compare to [8, Appendix]).

Example 3.18. Let $(C, L)$ be the pair of equations $C:\left(x_{1}\left(x_{0}^{2} x_{2}-x_{1}^{2}\right)^{2}=0\right)$ and $L:\left(x_{1}=0\right)$. The quintic $C$ is a double conic together with a secant line. Thus, $C$ is semistable and $\alpha=0$ (cf. Lemma 3.13). The line $L$ is a component of $C$ (it coincides with the secant line), but it does not destabilize the pair $(C, L)$ until $t=1$ (cf. Lemma 3.17). Thus the pair $(C, L)$ has interval of stability $[0,1]$, but $(C, L)$ is never stable (e.g. it has a $\mathbb{C}^{*}$-stabilizer). Note also that the orbit of $(C, L)$ is a minimal orbit in $X^{s s}(t)$ for $t \in(0,1)$. At $t=1$, the closure of the orbit of $(C, L)$ contains the orbit of the semistable pair $\left(C_{0}, L\right)$ with equation $C_{0}:\left(x_{0}^{2} x_{1} x_{2}^{2}=0\right)$.

On the other hand, the type of example mentioned above does not occur for $t>1$.

Lemma 3.19. For any $t>1$ which is not critical, we have $X^{s}(t)=X^{s s}(t)$. Thus, $\mathcal{M}(t)$ is a geometric quotient for all non-critical $t \in\left(1, \frac{5}{2}\right)$. 
3.3. Variation of GIT quotients for degree 5 pairs. One of the essential aspects of the theory of variation of GIT quotients of [37, 8] is the fact that the birational transformations that occur at a wall crossing are very explicit. Specifically, let $t$ be a critical slope (wall). We have $X^{s s}(t \pm \epsilon) \subsetneq X^{s s}(t)$. These inclusions induce contraction maps $\mathcal{M}(t \pm \epsilon) \rightarrow \mathcal{M}(t)$, whose common center $Z$ is the GIT quotient of $X^{s s}(t) \backslash\left(X^{s s}(t+\epsilon) \cup X^{s s}(t-\epsilon)\right)$ by $G$. Similarly, the exceptional sets $E_{ \pm}$of these birational maps are $\left(X^{s s}(t \pm \epsilon) \backslash X^{s s}(t \mp \epsilon)\right) / / G$. Furthermore, precise results about the local structure of these maps can be obtained by applying Luna's slice theorem. In particular, the case when the stabilizer of the minimal orbits at $t$ is $\mathbb{C}^{*}$ is well behaved (see [37, Thm. 5.6] and [8, Thm. 4.2.7]).

Ours is a very simple situation. Namely, for all critical slopes $t$ except 0,1 and $\frac{5}{2}$, the center $Z \subset \mathcal{M}(t)$ is a point and the two exceptional sets $E_{ \pm}$are weighted projective spaces (possibly modulo a finite group) of complementary dimension, i.e. $\operatorname{dim} E_{+}+\operatorname{dim} E_{-}=\operatorname{dim} \mathcal{M}(t)-1$. Furthermore, $E_{ \pm}$can be given a modular interpretation. This is due to the fact that, for each critical $t$ (except 0,1 and $\frac{5}{2}$ ), there is only one geometric situation relevant for the change of stability as illustrated by the following example:

Example 3.20. The change of stability at $t=\frac{5}{3}$ can be described as: the pairs $(C, L)$ with $C$ having a singularity of type $W_{12}$ become stable, and those with $L 5$-fold tangent to $C$ become unstable (cf. Proposition 3.11 and Lemma 3.15). The center $Z$ of the birational transformations at $t=\frac{5}{3}$ corresponds to the unique pair $(C, L)$ with $C$ having a singularity of type $W_{12}$ and $L$ being 5 -fold inflectional to $C$. Moreover, $E_{+}$can be interpreted as the stratum of $W_{12}$ occurring in the deformation of $N_{16}$, and $E_{-}$as the locus of quintics with a flex of order 5 .

The following proposition concludes our discussion.

Proposition 3.21. For each critical slope $t \in\left\{\frac{1}{7}, \frac{1}{4}, \frac{2}{5}, \frac{5}{8}, \frac{10}{7}, \frac{8}{5}, \frac{5}{3}, \frac{7}{4}, \frac{13}{7}, 2, \frac{11}{5}\right\}$ there exists a unique closed orbit $O\left(\left(C_{t}, L\right)\right) \subset X^{s s}(t) \backslash X^{s s}(t \pm \epsilon)$ with stabilizer $G_{\left(C_{t}, L\right)}^{0} \cong$ $\mathbb{C}^{*}$. The equations and the geometry of the pairs $\left(C_{t}, L\right)$ are described in Table 4 (see also the similar list in Wall [40]).

For the special critical slopes 0,1 and $\frac{5}{2}$, we have the following structural results. From Remark 2.13 and Corollary 2.18 it follows that the natural morphisms $\mathcal{M}(\epsilon) \rightarrow \mathcal{M}(0)$ and $\mathcal{M}\left(\frac{5}{2}-\epsilon\right) \rightarrow \mathcal{M}\left(\frac{5}{2}\right)$ are fibrations: they are generically a $\mathbb{P}^{2}-$ bundle and a weighted projective bundle respectively. The remaining case $t=1$ can be understood similarly to the cases when $t \neq 1$, but there are several issues that one has to consider. For example, one complication is that the centers of the birational transformations that occur at $t=1$ are curves (see Proposition [3.22), and thus we obtain weighted projective bundles for the exceptional loci (cf. 8, 37]). A more serious issue is that there exist points in $\mathcal{M}(1)$ such that the corresponding stabilizers are larger than $\mathbb{C}^{*}$ (e.g. III(2) of Proposition 3.22). One can still apply the Luna slice theorem to understand the local structure at those points, but the situation is slightly more complicated than in the $\mathbb{C}^{*}$ case.

3.4. Stability of degree 5 pairs at $t=1$. In constrast to the other critical slopes, the change of stability at $t=1$ is quite involved, as seen by inspecting the statements of $\$ 3.1$ and 33.2 . If we restrict to the pairs $(C, L)$ with $L$ generic the change of stability at $t=1$ becomes more conceptual: it corresponds to the division of singularities into three classes as given by Corollary 3.4 (see also Remark 3.25). 
TABLE 4 . The minimal orbits for the critical slopes $t \neq 0,1, \frac{5}{2}$

\begin{tabular}{|c|c|c|c|c|c|}
\hline$t$ & Equation for $C$ & Sing. at $p$ & Sing. at $p^{\prime}$ & mult $_{p^{\prime}}(C \cap L)$ & List [40 \\
\hline \hline$\frac{1}{7}$ & $x_{0}^{2} x_{2}^{3}+x_{0} x_{1}^{4}=0$ & $E_{6}$ & $A_{7}$ & $L$ is a comp. of $C$ & $(\mathrm{H} 2)$ \\
\hline$\frac{1}{4}$ & $x_{0}^{2} x_{1} x_{2}^{2}+x_{1}^{4} x_{2}=0$ & $D_{8}^{\prime}$ & $D_{5}$ & $L$ is a comp. of $C$ & $(\mathrm{H} 3)$ \\
\hline$\frac{2}{5}$ & $x_{0} x_{1}^{3} x_{2}+x_{0}^{2} x_{2}^{3}=0$ & $E_{7}$ & $A_{5}$ & $L$ is a comp. of $C$ & $(\mathrm{H} 1)$ \\
\hline$\frac{5}{8}$ & $x_{0}^{2} x_{2}^{3}+x_{1}^{5}=0$ & $E_{8}$ & $A_{4}$ & 5 & $(\mathrm{H} 5)$ \\
\hline$\frac{10}{7}$ & $x_{0} x_{1} x_{2}^{3}+x_{1}^{5}=0$ & $Z_{11}$ & $A_{1}$ & 5 & $(\mathrm{H} 14)$ \\
\hline$\frac{8}{5}$ & $x_{0} x_{1} x_{2}^{3}+x_{1}^{4} x_{2}=0$ & $Z_{12}$ & $A_{1}$ & 4 & $(\mathrm{H} 10)$ \\
\hline$\frac{5}{3}$ & $x_{0} x_{2}^{4}+x_{1}^{5}=0$ & $W_{12}$ & smooth & 5 & $(\mathrm{H} 13)$ \\
\hline$\frac{7}{4}$ & $x_{0}^{2} x_{2}^{3}+x_{1}^{3} x_{2}^{2}=0$ & double line & $A_{2}$ & 3 & - \\
\hline$\frac{13}{7}$ & $x_{0} x_{1} x_{2}^{3}+x_{1}^{4} x_{2}=0$ & $W_{13}$ & smooth & 4 & $(\mathrm{H} 9)$ \\
\hline 2 & $x_{0} x_{1} x_{2}^{3}+x_{1}^{3} x_{2}^{2}=0$ & double line & $A_{1}$ & 3 & - \\
\hline$\frac{11}{5}$ & $x_{0} x_{2}^{4}+x_{1}^{3} x_{2}^{2}=0$ & double line & smooth & 3 & - \\
\hline
\end{tabular}

For us, the most important aspect about the slope $t=1$ is the fact noted in Corollary 3.2 namely that the pairs $(C, L)$ with $C+L$ defining a sextic with simple singularities are GIT stable. Thus, we can define a moduli space of such pairs as the geometric quotient:

$$
\mathcal{M}:=\{(C, L) \mid C+L \text { has at worst simple singularities }\} / \text { (proj. equiv.) }
$$

The space $\mathcal{M}$ is a quasi-projective variety, compactified by the GIT quotient $\mathcal{M}(1)$. The boundary components of this compactification are given by the following result.

Proposition 3.22. The boundary $\mathcal{M}(1) \backslash \mathcal{M}$ consists of four one-dimensional components and two zero-dimensional components as described below:

Zero-Dimensional Components:

III(1) The point corresponding to the closed orbit of the pair $(C, L)$ with equations $L:\left(x_{0}=0\right)$ and $C:\left(x_{2}\left(x_{0} x_{2}-x_{1}^{2}\right)^{2}=0\right)$, and

III(2) The point corresponding to the closed orbit of the pair $(C, L)$ with equations $L:\left(x_{0}=0\right)$ and $C:\left(x_{0} x_{1}^{2} x_{2}^{2}=0\right)$.

One-Dimensional Components:

II(1) The rational curve parameterizing the orbits of the pairs $\left(C_{\lambda}, L\right)$ with equations $L:\left(x_{0}=0\right)$ and $C_{\lambda}:\left(x_{2}\left(x_{0} x_{2}-x_{1}^{2}\right)\left(x_{0} x_{2}-\lambda x_{1}^{2}\right)=0\right)$, where $\lambda \neq 0,1, \infty$,

II(2a) The rational curve parameterizing the orbits of the pairs $\left(C_{\lambda}, L\right)$ with equations $L:\left(x_{0}=0\right)$ and $C_{\lambda}:\left(x_{0} x_{1} x_{2}\left(x_{2}-x_{1}\right)\left(x_{2}-\lambda x_{1}\right)=0\right)$, where $\lambda \neq 0,1, \infty$,

$\mathrm{II}(2 \mathrm{~b})$ The rational curve parameterizing the orbits of the pairs $\left(C_{\lambda}, L\right)$ with equations $L:\left(x_{1}=0\right)$ and $C_{\lambda}:\left(x_{0}^{2} x_{2}\left(x_{2}-x_{1}\right)\left(x_{2}-\lambda x_{1}\right)=0\right)$, where $\lambda \neq 0,1, \infty$, and

II(3) The rational curve parameterizing the orbits of the pairs $\left(C_{\lambda}, L\right)$ with equations $L:\left(x_{1}=0\right)$ and $C_{\lambda}:\left(\left(\lambda x_{0}-(\lambda+1) x_{1}+x_{2}\right)\left(x_{0} x_{2}-x_{1}^{2}\right)^{2}\right.$ $=0)$, where $\lambda \neq 0,1, \infty$. 
The incidence relations are given in Figure 2, All the components except II(3) are strict GIT boundary components (i.e. parameterizes strictly semistable pairs). The stabilizer for the type II boundary components (except II(3)) is $\mathbb{C}^{*}$.

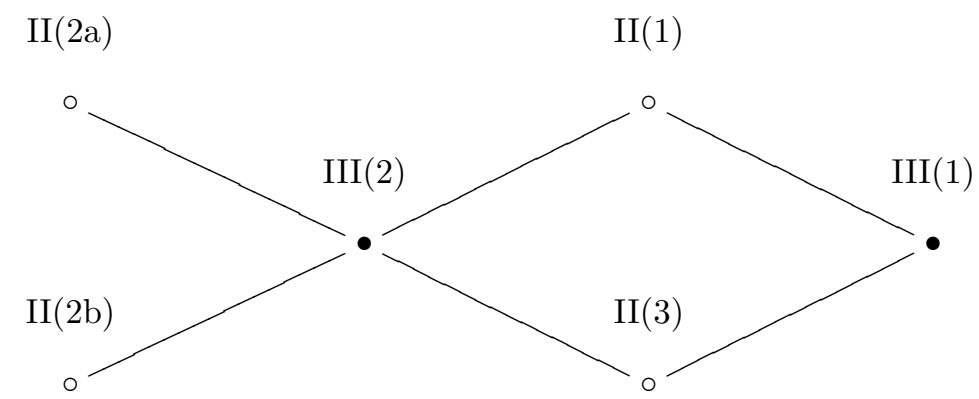

FIGURE 2. Incidence diagram of the boundary components

Remark 3.23. The labeling of the boundary components is in accordance with the similar list of Shah for plane sextics ([35, Thm. 2.4]). Some of the boundary components in Shah's list do not occur in our situation (e.g. II(4) and IV), and the case II(2) splits into two subcases (depending on the relative position of the line $L)$.

We note that there exists a close relationship between the boundary components of $\mathcal{M} \subset \mathcal{M}(1)$ and the simple elliptic and cusp singularities adjacent to $N_{16}$. A similar situation was observed by Brieskorn [6] for the case of the triangle singularities. He noticed that there exists a natural matching between the Baily-Borel compactification of a certain period domain $\mathcal{D} / \Gamma$ and the simple elliptic and cusp singularities adjacent to the given triangle singularity. Furthermore, the incidence diagram of the boundary components coincides with the adjacency diagram for the corresponding singularities. This is also the case in our situation. Here we note this for the GIT compactification. By the results of section 4, $\mathcal{M}(1)$ can be interpreted as a Baily-Borel compactification of an appropriate $\mathcal{D} / \Gamma$. Thus, the situation for $N_{16}$ is completely analogous to that for the triangle singularities.

Proposition 3.24. Assume that $(C, L)$ is a semistable pair at $t=1$ such that $C$ has at least one non-simple singularity. Assume also that $L$ is generic. Then, the image $x \in \mathcal{M}(1) \backslash \mathcal{M}$ of the pair under the natural projection map $X^{s s}(1) \rightarrow \mathcal{M}(1)$ satisfies:

i) If $C$ has a simple elliptic singularity of type $\widetilde{E_{7}}\left(\widetilde{E_{8}}\right)$, then $x$ belongs to the boundary component II(2a) (resp. II(1)).

ii) If $C$ has a cusp singularity of type $T_{2 q r}$ with $4 \leq q \leq r \leq 6$ ( $T_{23 k}$ with $7 \leq k \leq 10$ ), then $x$ is the point III(2) (resp. III(1)).

iii) If $C$ is non-reduced, then $x$ belongs to one of the components II(2b), II(3), $\operatorname{III}(1)$ or $\operatorname{III}(2)$.

In particular, there exists a natural matching between the simple elliptic and cusp singularities adjacent to $N_{16}$ and the boundary components of $\mathcal{M}(1)$ as given in Figure 3, 


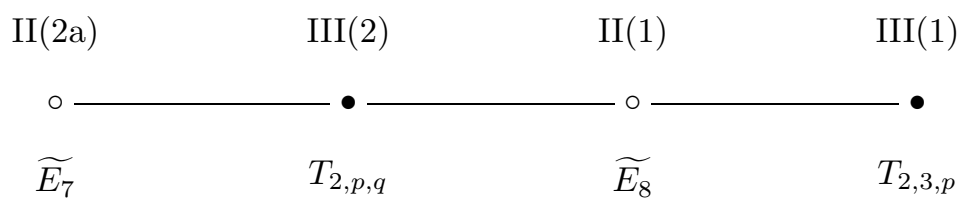

Figure 3 . The simple elliptic and cusp singularities adjacent to $N_{16}$

Remark 3.25. We close by noting the following explanation for the division of singularities given by Corollary 3.4. According to Theorem 2.5. the semistability at $t=1$ of the pair $(C, L)$ is roughly equivalent to the pair $\left(\mathbb{P}^{2}, \frac{1}{2}(C+L)\right)$ being $\log$ canonical. Let $S$ be the double cover of $\mathbb{P}^{2}$ branched along $C+L$. From [16, Prop. 5.20], it follows that $\left(\mathbb{P}^{2}, \frac{1}{2}(C+L)\right)$ is $\log$ canonical if and only if $S$ is $\log$ canonical. The classification of canonical and log canonical singularities is given by [16. Ch. 4]: the simple (du Val) singularities are canonical, and the simple elliptic and cusp singularities are strictly $\log$ canonical. The singularities of $S$ are in one-to-one correspondence (including the type) with the singularities of $C+L$. Since for $d=5$ and $t=1$, the converse of Theorem 2.5 also holds we obtain the characterization of Corollaries 3.2 and 3.4 for the stability at $t=1$.

\section{Moduli of Pairs via $K 3$ SURFACES}

An alternative construction of the moduli space of degree 5 pairs is obtained via the periods of $K 3$ as follows. To a generic degree 5 pair $(C, L)$ we associate the surface $S_{(C, L)}$ obtained as a double cover of $\mathbb{P}^{2}$ along the sextic $C+L$. The minimal desingularization $\widetilde{S}_{(C, L)}$ of $S_{(C, L)}$ is a degree two $K 3$ surface. It is easy to see that $\widetilde{S}_{(C, L)}$ is an $M$-polarized $K 3$ surface in the sense of Nikulin and Dolgachev (see [7]), where $M$ is the rank 6 hyperbolic lattice spanned by the polarization class and the exceptional divisors. It follows that the natural period map gives a birational isomorphism between the moduli space of degree 5 pairs and the moduli space $\mathcal{D} / \Gamma$ of $M$-polarized $K 3$ surfaces. This construction works in fact for all pairs $(C, L)$ such that $C+L$ has at worst simple singularities. As a consequence, we obtain:

Theorem 4.1. The birational map which associates to a degree 5 pair $(C, L)$ the periods of the $K 3$ surface $\widetilde{S}_{(C, L)}$ extends to an isomorphism $\mathcal{P}: \mathcal{M} \rightarrow \mathcal{D} / \Gamma$.

We recall that $\mathcal{M}$ denotes the moduli space of degree 5 pairs $(C, L)$ satisfying the condition that the sextic $C+L$ has at worst simple singularities. The space $\mathcal{M}$ is constructed as a geometric quotient, and it is compactified by the GIT quotient $\mathcal{M}(1)$. As mentioned above, $\mathcal{D} / \Gamma$ is the moduli space of $M$-polarized $K 3$ surfaces. The precise definitions are given in $\$ 4.2$ below. The proof of the theorem then follows from standard results on $K 3$ surfaces and the analysis of the geometric meaning of the notion of $M$-polarization. The details are given in $\$ 4.3$. We note additionally that it follows automatically from work of Looijenga 20, that the isomorphism of Theorem 4.1 extends to the boundary.

Theorem 4.2. The period map $\mathcal{P}: \mathcal{M} \rightarrow \mathcal{D} / \Gamma$ extends to an isomorphism of projective varieties $\overline{\mathcal{P}}: \mathcal{M}(1) \stackrel{\cong}{\longrightarrow}(\mathcal{D} / \Gamma)^{*}$, where $(\mathcal{D} / \Gamma)^{*}$ denotes the Baily-Borel compactification of $\mathcal{D} / \Gamma$. 
The Baily-Borel compactification and the matching of the boundary components given by the above theorem are discussed in $\$ 4.4$

4.1. Standard notation and facts about lattices and $K 3$ surfaces. By a lattice we understand a free $\mathbb{Z}$-module $L$ together with a symmetric bilinear form, which we denote by $x . y$ for $x, y \in L$. The basic invariant of a lattice is its signature. In particular, if the signature is $(1, n)$ we call the lattice hyperbolic. A lattice is even if $x . x \equiv 0 \bmod 2$ for every $x \in L$. The direct sum of lattices is always assumed orthogonal. For a lattice embedding $M \subseteq L, M_{L}^{\perp}$ denotes the orthogonal complement of $M$ in $L$.

The following standard lattices are used frequently: the root lattices $A_{n}$ (for $n \geq$ 1 ), $D_{m}$ (for $m \geq 4$ ), and $E_{r}$ (for $\left.r=6,7,8\right)$, the hyperbolic plane $U$, the parabolic lattices (negative semidefinite) $\widetilde{A}_{n}, \widetilde{D}_{m}$, and $\widetilde{E}_{r}$, and the hyperbolic lattices $T_{p, q, r}$ for $\frac{1}{p}+\frac{1}{q}+\frac{1}{r}<1$. Given $L$ a lattice, $L(n)$ denotes the lattice with the same underlying $\mathbb{Z}$-module as $L$ but with the bilinear form multiplied by $n$.

Notation 4.3. Let $L$ be an even lattice. We define:

- $\operatorname{div}(x)$ : the positive integer $d$ such that $x . L \cong d \mathbb{Z}$;

- $L^{*}:=\{y \in L \otimes \mathbb{Q} \mid x . y \in \mathbb{Z}$ for all $x \in L\}$ : the dual lattice;

- $A_{L}=L^{*} / L$ : the discriminant group endowed with the induced finite quadratic form $q_{L}$;

- $O(L)$ : the group of isometries of $L$;

- $O\left(q_{L}\right)$ : the automorphisms of $A_{L}$ that preserve the quadratic form $q_{L}$;

- $O_{-}(L)$ : the group of isometries of $L$ of spinor norm 1;

- $\widetilde{O}(L)$ : the group of isometries of $L$ that induce the identity on $A_{L}$;

- $O^{*}(L):=O_{-}(L) \cap \widetilde{O}(L)$;

- $\Delta(L)$ : the set of roots of $L$, where $\delta \in L$ is a root if $\delta^{2}=-2$;

- $\widetilde{\Delta}(L)$ : the set of generalized roots of $L$, where $\delta \in L$ is a generalized root if $\delta^{2}=-k$ and $\operatorname{div}(\delta)=\frac{k}{2}$ for some even positive integer $k$;

- $W(L)$ (and $\widetilde{W}(L))$ : the (generalized) Weyl group, i.e. the group of isometries generated by reflections $s_{\delta}$ in (generalized) roots $\delta$, where

$$
s_{\delta}(x)=x-2 \frac{x . \delta}{\delta^{2}} \delta .
$$

Definition 4.4. Given two lattices $L$ and $L^{\prime}$ and a lattice embedding $L \hookrightarrow L^{\prime}$, we call it a primitive embedding iff $L^{\prime} / L$ is a free $\mathbb{Z}$-module. Equivalently, the embedding is primitive iff $\operatorname{Sat}(L):=\left\{y \in L^{\prime} \mid n y \in L\right.$ for some positive integer $\left.n\right\}$ coincides with $L$.

For a surface $S$, the intersection form gives a natural lattice structure on the torsion-free part of $H^{2}(S, \mathbb{Z})$ and on the Neron-Severi group $\mathrm{NS}(S)$. For a $K 3$ surface, we have $H^{1}\left(S, \mathcal{O}_{S}\right)=0$, and we identify $\operatorname{Pic}(S) \cong \mathrm{NS}(S)$. Both $H^{2}(S, \mathbb{Z})$ and $\operatorname{Pic}(S)$ are torsion free. The natural map $\operatorname{Pic}(S) \stackrel{c_{1}}{\longrightarrow} H^{2}(S, \mathbb{Z})$ is a primitive lattice embedding. By the Hodge index theorem, $\operatorname{Pic}(S)$ is a hyperbolic lattice.

Notation 4.5. If $S$ is a $K 3$ surface, we use $O(S), W(S), \Delta(S)$, etc. to denote the corresponding objects associated to the lattice $\operatorname{Pic}(S)$. We also use:

- $\Delta^{+}(S)$ for the set of effective $(-2)$ divisor classes in $\operatorname{Pic}(S)$;

- $V^{+}(S) \subset S_{\mathbb{R}}$ : the Kähler cone; 
- $C^{+}(S)=\left\{x \in \operatorname{Pic}(S) \cap V^{+}(S) \mid x . \delta>0\right.$ for all $\left.\delta \in \Delta^{+}(S)\right\}$ : the ample cone.

We note that, on a $K 3$ surface, any $x \in \operatorname{Pic}(S)$ with $x . x \geq-2$ has the property that either $x$ or $-x$ is effective. In particular, $\Delta(S)=\Delta^{+}(S) \sqcup\left(-\Delta^{+}(S)\right)$.

Notation 4.6. Unless specified otherwise, the symbols $\Lambda, M$ and $T$ will denote throughout the chapter the lattices $E_{8}^{\oplus 2} \oplus U^{\oplus 3}, D_{4} \oplus U(2)$ and $D_{4} \oplus E_{8} \oplus U \oplus U(2)$ respectively. The lattice $\Lambda$ is the unique even, unimodular lattice of signature $(3,19) ; \Lambda$ is isometric to $H^{2}(S, \mathbb{Z})$ for any $K 3$ surface $S$. The lattices $M$ and $T$ have signature $(1,5)$ and respectively $(2,14)$ and they can be embedded in $\Lambda$ such that they are mutually orthogonal.

Definition 4.7. A polarization for a $K 3$ surface is the class of a nef and big divisor $H$. The degree of the polarization (and of the surface) is $H^{2}$.

We use the following standard results on $K 3$ surfaces: the global Torelli theorem, the surjectivity of the period for algebraic $K 3$ surfaces, and the results on linear systems on a $K 3$ surface. We recall the following theorem of Mayer ([11, Thm. 27]).

Theorem 4.8 (Mayer's Theorem). Let $H$ be a nef and big divisor on the K3 surface $S$. Then $|H|$ has a base point iff $|H|$ has a fixed curve iff $H=k E+R$ (linearly equivalent), where $E$ is a smooth elliptic curve, $R$ is a smooth rational curve, $R . E=1$, and $k \geq 2$.

We note also the following easy converse ([23, Prop. 1, p. 35]).

Proposition 4.9. Let $D$ be a big and nef divisor on a K3 surface. Assume that there exists a divisor $E$ such that D.E $=1$ and $E^{2}=0$. Then the linear system $|D|$ has a fixed component.

4.2. The $K 3$ surface associated to a degree 5 pair and the period map. Let $(C, L)$ be a degree 5 pair such that $C+L$ is a sextic with at worst simple singularities, and let $\widetilde{S}_{(C, L)}$ be the associated degree two $K 3$ surface. We are concerned here with establishing the basic properties of these types of surfaces, and finding a moduli space for them. We proceed in three basic steps. First, by considering the generic case, we see that $\widetilde{S}_{(C, L)}$ is naturally $M$-polarized for a certain lattice $M$. Then, we establish some basic arithmetic properties about $M$ and its orthogonal complement $T:=M_{\Lambda}^{\perp}$ and determine the period domain $\mathcal{D} / \Gamma$. Finally, we show that the construction can be extended to the non-generic case.

4.2.1. The Picard lattice of $\widetilde{S}_{(C, L)}$. In this subsection, we assume that $(C, L)$ is a generic degree 5 pair. In particular, $C$ is smooth and $L$ is a transversal. The $K 3$ surface $\widetilde{S}_{(C, L)}$ is obtained as the desingularization of $S_{(C, L)}$, the double cover of $\mathbb{P}^{2}$ along $C+L$. Let $\pi: \widetilde{S}_{(C, L)} \rightarrow \mathbb{P}^{2}$ be the natural projection, and $h=\pi^{*} l \in$ $\operatorname{Pic}\left(\widetilde{S}_{(C, L)}\right)$ the pullback of the class of a line from $\mathbb{P}^{2}$. Since the surface $S_{(C, L)}$ has five ordinary double points coming from the intersection $C \cap L$, it follows that $\operatorname{Pic}\left(\widetilde{S}_{(C, L)}\right)$ contains five additional classes $e_{1}, \ldots, e_{5}$ corresponding to the exceptional divisors of $\widetilde{S}_{(C, L)} \rightarrow S_{(C, L)}$. By construction we have that $h$ is a degree 2 polarization for $\widetilde{S}_{(C, L)}$ and the intersection numbers $h \cdot e_{i}=0$ and $e_{i} \cdot e_{j}=-2 \delta_{i j}$. 
Finally, since $L$ is in the branch locus of $\pi$, the surface $\widetilde{S}_{(C, L)}$ contains another rational curve, namely the inverse image $L^{\prime}$ of the line $L$. In conclusion, we obtain:

Lemma 4.10. Let $\widetilde{S}_{(C, L)}$ be the $K 3$ surface associated to a generic degree 5 pair $(C, L)$. Then $\widetilde{S}_{(C, L)}$ contains 6 irreducible $(-2)$-curves, whose classes $l^{\prime}, e_{1}, \ldots, e_{5}$ satisfy $l^{\prime} . e_{i}=1$ and $e_{i} . e_{j}=0$ for $i \neq j$. Additionally, the structural morphism $\pi: \widetilde{S}_{(C, L)} \rightarrow \mathbb{P}^{2}$ is given by the class $h:=2 l^{\prime}+e_{1}+\cdots+e_{5}$.

As a consequence of the previous lemma, it follows that the Picard lattice of $\widetilde{S}_{(C, L)}$ contains the sublattice spanned by the classes $l^{\prime}, e_{1}, \ldots, e_{5}$. We will see that generically $\operatorname{Pic}\left(\widetilde{S}_{(C, L)}\right)$ coincides with this sublattice. Conversely, we will prove that this condition characterizes the surfaces $\widetilde{S}_{(C, L)}$. To proceed, we need to fix the following notation (compatible with Notation 4.6).

Notation 4.11. We denote by $M$ the abstract rank 6 lattice spanned by $l^{\prime}, e_{1}, \ldots, e_{5}$ with the intersection form given by: $l^{\prime 2}=e_{i}^{2}=-2, l^{\prime} \cdot e_{i}=1$ and $e_{i} \cdot e_{j}=0$ for $i \neq j$. The basis $\left\{l^{\prime}, e_{1}, \ldots, e_{5}\right\}$ of $M$ is assumed fixed. We denote

$$
h:=2 l^{\prime}+e_{1}+\cdots+e_{5}
$$

and $f_{i}:=h-e_{i}$ for $i=1, \ldots, 5$. In particular, $h^{2}=2, h . l^{\prime}=1$, and $h . e_{i}=0$.

Proposition 4.12. Let $(C, L)$ be a generic degree 5 pair and $j: M \rightarrow \operatorname{Pic}\left(\widetilde{S}_{(C, L)}\right)$ the lattice embedding given by Lemma 4.10. Then $j$ is a primitive lattice embedding.

Proof. Assume that $j$ is not primitive. Then the embedding $j$ factors as

$$
M \subsetneq \operatorname{Sat}(M) \hookrightarrow \operatorname{Pic}\left(\widetilde{S}_{(C, L)}\right),
$$

where $\operatorname{Sat}(M)$ is the saturation of the lattice $M$ in $\operatorname{Pic}\left(\widetilde{S}_{(C, L)}\right)$. Thus, $\operatorname{Sat}(M)$ is a non-trivial overlattice of $M$ and as such is classified by a non-trivial isotropic subgroup of $A_{M}$ ([27, Sect. 4]). The discriminant group $A_{M}=M^{*} / M \cong(\mathbb{Z} / 2 \mathbb{Z})^{4}$ is generated by $\left\{f_{i}^{*} \mid i=1, \ldots 5\right\}$, where $f_{i}^{*}$ is the class of $f_{i} / \operatorname{div}\left(f_{i}\right)\left(\right.$ N.B. $\operatorname{div}\left(f_{i}\right)=2$ and the notation is that of Notations 4.3 and 4.11). It is easy to see that the only non-trivial isotropic elements of $A_{M}$ (for the induced quadratic form) are precisely the elements $f_{i}^{*}$. It follows that $M \neq \operatorname{Sat}(M)$ is equivalent to some $f_{i}$ being 2divisible in $\operatorname{Pic}\left(\widetilde{S}_{(C, L)}\right)$. We have $f_{i}=2 f_{i}^{\prime}$ for some $f_{i}^{\prime} \in \operatorname{Pic}\left(\widetilde{S}_{(C, L)}\right)$ and then

$$
h=f_{i}+e_{i}=2 f_{i}^{\prime}+e_{i} .
$$

By Proposition 4.9 it follows that $h$ is not base point free. This is a contradiction to the fact that $h$ defines the morphism $\pi: \widetilde{S}_{(C, L)} \rightarrow \mathbb{P}^{2}$.

Remark 4.13. More generally, let $S$ be any $K 3$ surface. The same argument as above gives the following statement. If $j: M \rightarrow \operatorname{Pic}(S)$ is a lattice embedding with $j(h) \in \operatorname{Pic}(S)$ a base point free polarization, then $j$ is primitive.

Proposition 4.14. Assume that $S$ is a $K 3$ surface such that $\operatorname{Pic}(S)$ is isometric to the lattice $M$. Then $S$ is the double cover of $\mathbb{P}^{2}$ branched over a reducible sextic $C+L$. Moreover, $C$ is a smooth quintic and $L$ is a line intersecting $C$ transversely.

Proof. By assumption there exist $h, l^{\prime}, e_{1}, \ldots, e_{5} \in \operatorname{Pic}(S)$ satisfying the numerical conditions from Notation 4.11. There is no loss of generality in assuming that $h$ is nef (if not, this can be achieved by acting by $\pm W(S)$ ). By acting with the 
reflections $s_{e_{i}}$ (i.e. change $e_{i}$ to $-e_{i}$ ) we can further assume that the classes $e_{i}$ are effective.

The class $h$ defines a degree two polarization for $S$. We claim that $h$ is base point free. If not, by Mayer's theorem, we get that $h=2 d+r$ with $d^{2}=0$ and $d . h=1$ for some $d, r \in \operatorname{Pic}(S) \cong M$. This gives a contradiction. Namely, we write:

$$
d=a l^{\prime}+b_{1} e_{1} \cdots+b_{5} e_{5}
$$

for some integers $a, b_{1}, \ldots, b_{5}$. The condition $d . h=1$ gives $a=1$, and then

$$
d^{2}=-2+2\left(b_{1}+\cdots+b_{5}\right)-2\left(b_{1}^{2}+\cdots+b_{5}^{2}\right) \equiv 2 \bmod 4,
$$

contradicting the assumption that $d^{2}=0$. In conclusion, the linear system defined by $h$ gives a degree two map $\pi: S \rightarrow \mathbb{P}^{2}$ branched along a sextic $B$.

Since $\langle h\rangle_{M}^{\perp} \cap \Delta(M)=\left\{ \pm e_{1}, \ldots, \pm e_{5}\right\}$, it follows that $e_{1}, \ldots, e_{5}$ are classes of irreducible rational curves $E_{1}, \ldots, E_{5}$. These curves are contracted by $\pi$ to five ordinary double points for the sextic $B$. Let $L^{\prime}$ be the curve corresponding to $l^{\prime}$ and $L:=\pi\left(L^{\prime}\right)$. Since $l^{\prime} \cdot h=1$, the projection formula gives that $L$ is a line. Moreover, since $l^{\prime} \cdot e_{i}=1, L$ has to pass through all 5 singular points of the branch curve $B$. By Bezout, the only possibility is that $L$ is a component of $B$.

From the previous two propositions, it follows that generically $\operatorname{Pic}\left(\widetilde{S}_{(C, L)}\right) \cong M$. Here, generically should be understood in the sense of moduli; i.e. it holds on the complement of the union of a countable number of proper subvarieties.

Corollary 4.15. Assume that $(C, L)$ is a sufficiently general degree 5 pair. Then there exists a lattice isometry $M \cong \operatorname{Pic}\left(\widetilde{S}_{(C, L)}\right)$.

Without the genericity assumption we obtain the following statement.

Lemma 4.16. Let $(C, L)$ be a degree 5 pair such that $C$ has at worst simple singularities and $L$ intersects $C$ transversely. Then there exists a primitive embedding $j: M \hookrightarrow \operatorname{Pic}\left(\widetilde{S}_{(C, L)}\right)$ such that $j(h)$ is a base point free degree 2 polarization.

Proof. Since we assume transversal intersection, the construction of Lemma 4.10 applies ad litteram. In particular, the class $j(h)$ defines the morphism $\pi: \widetilde{S}_{(C, L)} \rightarrow$ $\mathbb{P}^{2}$, and thus it is base point free. The embedding $j$ primitive by Remark 4.13 .

4.2.2. $M$-polarized K3 surfaces and the period map. The statements of Corollary 4.15 and Lemma 4.16 say that the $K 3$ surfaces $\widetilde{S}_{(C, L)}$ associated to a degree 5 pair $(C, L)$ are characterized by the fact that they are $M$-polarized $K 3$ surfaces.

Definition 4.17. Let $M$ be the lattice defined in Notation 4.11. An $M$-polarized $K 3$ surface is a pair $(S, j)$ such that $j: M \hookrightarrow \operatorname{Pic}(S)$ is a primitive lattice embedding. The embedding $j$ is called the $M$-polarization of $S$. If the polarization is understood, we simply say $S$ is an $M$-polarized $K 3$ surface.

The lattice $M$ admits a unique primitive embedding into the $K 3$ lattice $\Lambda$.

Lemma 4.18. Let $M$ be as in Notation 4.11. Then $M$ is isometric to the lattice $D_{4} \oplus U(2)$ and admits a unique primitive embedding $M \hookrightarrow \Lambda$ into the K3 lattice $\Lambda$. The orthogonal complement $T:=M_{\Lambda}^{\perp}$ with respect to this embedding is isometric to $D_{4} \oplus E_{8} \oplus U \oplus U(2)$. 
Proof. The isometry $M \cong D_{4} \oplus U(2)$ is given by taking $\left\{l^{\prime}, e_{1}, e_{2}, e_{3}, f_{4}, f_{5}\right\}$ as a basis for $M$. The existence of the primitive embedding $M \hookrightarrow \Lambda$ follows from [27. Thm. 1.14.4]. The uniqueness is essentially equivalent to the fact that $M$ and its orthogonal complement $T$ are uniquely determined by their invariants (i.e. signature and discriminant group). Since $M$ and $T$ are indefinite lattices, the latter statement follows from [27, Thm. 1.10.1].

It is a standard fact (see [7]) that the moduli space of $M$-polarized $K 3$ surfaces is a quotient $\mathcal{D} / \Gamma$ for a certain bounded symmetric domain $\mathcal{D}$ of type IV and a certain arithmetic group $\Gamma$. Namely, the condition of the $M$-polarization determines a tower of primitive embeddings

$$
M \hookrightarrow \operatorname{Pic}(S) \hookrightarrow H^{2}(S, \mathbb{Z}) \cong \Lambda .
$$

This implies that the period of an $M$-polarized $K 3$ surface belongs to the following subdomain of the period domain of $K 3$ surfaces:

$$
\mathcal{D}=\{\omega \in \mathbb{P}(\Lambda \otimes \mathbb{C}) \mid \omega \cdot \omega=0, \omega \cdot \bar{\omega}>0, \text { and } \omega \perp M\}_{0}
$$

(the (unique) embedding $M \hookrightarrow \Lambda$ is assumed fixed). Conversely, since $\operatorname{Pic}(S) \cong$ $H^{2}(S, \mathbb{Z}) \cap H^{1,1}(S)$, every point of $\mathcal{D}$ corresponds to an $M$-polarized $K 3$ surface. Let $T=M_{\Lambda}^{\perp}$ be the orthogonal complement of $M$ (called the transcendental lattice). It is convenient to identify $\mathcal{D}$ to the domain

$$
\{\omega \in \mathbb{P}(T \otimes \mathbb{C}) \mid \omega \cdot \omega=0, \omega \cdot \bar{\omega}>0\}_{0} .
$$

In particular, note the natural action of the groups $O^{*}(T)$ and $O_{-}(T)$ on $\mathcal{D}$.

To specify the moduli of $M$-polarized $K 3$ surfaces it suffices to determine the arithmetic group $\Gamma$. In the standard situation considered in [7] one requires that the $M$-polarization is pointwise fixed by the group $\Gamma$, and thus takes $\Gamma$ to be $O^{*}(T)$. In our geometric context we have to require less, namely $\Gamma$ should fix (not necessarily pointwise) $M$ and the element $h \in M$. Thus, we make the following definition for $\mathcal{D}$ and $\Gamma$. The reason for this choice is explained in Proposition 4.22 below.

Notation 4.19. Fix the primitive embedding $M \hookrightarrow \Lambda$ and let $T:=M_{\Lambda}^{\perp}$. We define $\mathcal{D}$ to be the domain given by (4.1), and $\Gamma:=O_{-}(T)$.

To explain the choice of $\Gamma$ and to see that indeed $\mathcal{D} / \Gamma$ is a moduli space for degree 5 pairs, we note the following properties of the lattices $M$ and $T$.

Lemma 4.20. Let $\left\{l^{\prime}, e_{1}, \ldots, e_{5}\right\}$ be the standard basis of $M$ and regard $\Sigma_{5}$ as the subgroup of $O(M)$ which permutes the basis elements $\left\{e_{1}, \ldots, e_{5}\right\}$. Then, the composition $\Sigma_{5} \rightarrow O(M) \rightarrow O\left(q_{M}\right)$ induces an isomorphism $\Sigma_{5} \rightarrow O\left(q_{M}\right)$. A similar statement holds for $T$.

The lemma establishes the following relation between the arithmetic groups that occur in the construction of the moduli space for $M$-polarized $K 3$ surfaces.

Corollary 4.21. Let $T$ be as above. Then $O_{-}(T)=O^{*}(T) \rtimes \Sigma_{5}$.

To understand the geometric meaning of the previous corollary, one has to investigate the relation of these groups to the (generalized) Weyl group (see Notation 4.3). 
Proposition 4.22. Let $T$ be the lattice of Notation 4.6. Then

i) $O^{*}(T)=W(T)$,

ii) $O_{-}(T)=\widetilde{W}(T)$.

Geometrically, $O^{*}(T)$ is the local monodromy group of the singularity $N_{16}, O_{-}(T)$ is the monodromy group for the degree 5 pairs $(C, L)$, and $\Sigma_{5} \cong O_{-}(T) / O^{*}(T)$ is the monodromy at infinity (i.e. it acts on the intersection $C \cap L$ ).

Proof. It is easy to verify that the lattice $T$ is isometric to the Milnor lattice of the singularity $N_{16}$. For example any singularity of class $N_{16}$ has a $\mu$-constant deformation to the special case $x^{5}+y^{5}+z^{2}$. For this case, the Milnor lattice can be computed by the Thom-Sebastiani theorem (see 3]). By a theorem of Ebeling (9, Thm. 5.5]) it follows that the local monodromy group coincides with $O^{*}(T)$ (see also [29]). The monodromy group is generated by Picard-Lefschetz transformations (reflections) in the vanishing cycles. Thus, $O^{*}(T)=W(T)$.

For the second part, we note $T \cong M \oplus E_{8} \oplus U$. The residual $\Sigma_{5} \cong O_{-}(T) / O^{*}(T)$ corresponds to the permutation of the basis elements $\left\{e_{1}, \ldots, e_{5}\right\}$ in the $M$ summand. We note that the reflection in the generalized root $\delta=e_{i}-e_{j}$ transposes $e_{i}$ and $e_{j}$ and leaves the other basis elements invariant (including $l^{\prime}$ ). Since $\widetilde{W}(T) \subseteq O_{-}(T)$ always holds, we conclude $O_{-}(T)=\widetilde{W}(T)$.

Let $U \subset X \cong \mathbb{P}^{N} \times \mathbb{P}^{2}$ be the open subset parameterizing degree 5 pairs $(C, L)$ with $C$ smooth and $L$ a transversal. Fix a base point $x \in U$ corresponding to a pair $(C, L)$. Then there exists a natural monodromy action of $\pi_{1}(U, x)$ on $H^{2}\left(\widetilde{S}_{(C, L)}, \mathbb{Z}\right)$. For obvious geometric reasons we have that the monodromy group $\Gamma$ satisfies:

$$
\Gamma:=\operatorname{Im}\left(\pi_{1}(U, x) \rightarrow \operatorname{Aut}\left(H^{2}\left(\widetilde{S}_{(C, L)}, \mathbb{Z}\right)\right)\right) \subseteq O_{-}(T) .
$$

The argument above shows that, in fact, we have equality. Namely, the reflections in vanishing cycles corresponding to the degenerations of $C$ generate $O^{*}(T) \subseteq \Gamma$. On the other hand, it is well known that $\Gamma$ acts as $\Sigma_{5}$ on the five points of the intersection $C \cap L$ (e.g. [13). By construction, a permutation of the intersection points gives a permutation of the basis elements $\left\{e_{1}, \ldots, e_{5}\right\}$ of $M \hookrightarrow \operatorname{Pic}\left(\widetilde{S}_{(C, L)}\right)$. Thus, indeed $\Gamma=O_{-}(T)$.

We can now conclude that the moduli space of degree 5 pairs $\mathcal{M}$ constructed by GIT is birationally equivalent to the moduli of $M$-polarized $K 3$ surfaces $\mathcal{D} / \Gamma$.

Corollary 4.23. The period map that associates to a generic degree 5 pair $(C, L)$ the periods of the $K 3$ surface $\widetilde{S}_{(C, L)}$ defines a birational map $\mathcal{P}: \mathcal{M} \rightarrow \mathcal{D} / \Gamma$.

Proof. Let $U$ be the open subset of $\mathcal{M}$ parameterizing the degree 5 pairs $(C, L)$ with $C$ smooth and with a transversal intersection $C \cap L$. Let $\widetilde{U}$ be the $\Sigma_{5}$-cover of $U$ that parameterizes triples $(C, L, \sigma)$, where $\sigma:\{1, \ldots, 5\} \rightarrow C \cap L$ is a labeling of the five points of intersection of $C$ and $L$. By the results of 4.2 .1 (esp. Lemma 4.16), the surface $\widetilde{S}_{(C, L)}$ carries a natural $M$-polarization $j: M \hookrightarrow \operatorname{Pic}\left(\widetilde{S}_{(C, L)}\right)$. Therefore, by [7], there is a well-defined map $\widetilde{\mathcal{P}}: \widetilde{U} \rightarrow \mathcal{D} / O^{*}(T)$ sending $(C, L, \sigma)$ to the periods of $\left(S_{(C, L)}, j\right)$. The two main theorems for $K 3$ surfaces (Torelli and surjectivity of the period map) together with Proposition 4.14 give that $\widetilde{\mathcal{P}}$ is a birational morphism. The map $\widetilde{\mathcal{P}}$ is clearly $\Sigma_{5}$-equivariant (see Proposition 4.22). Thus, it descends to a birational morphism $\mathcal{P}: U \rightarrow \mathcal{D} / \Gamma$ as needed. 
4.2.3. M-Polarizations for non-generic intersections. In order to conclude that we have a period map $\mathcal{P}: \mathcal{M} \rightarrow \mathcal{D} / \Gamma$ sending a degree 5 pair $(C, L)$ to the periods of $\widetilde{S}_{(C, L)}$, we need to address two points. Namely, we have to extend the construction of $M$ polarization to the case of a non-transversal intersection $C \cap L$, and we have to show that this construction fits in families.

The main problem in constructing an $M$-polarization in the case of non-transversal intersections is the fact that the Picard lattice $\operatorname{Pic}\left(\widetilde{S}_{(C, L)}\right)$ acquires additional $(-2)$ classes. A priori it is not clear which of those classes should be chosen as $l^{\prime}, e_{1}, \ldots, e_{5}$ (see Notation 4.11). For example, the reader is encouraged to consider the case when $L$ becomes simply tangent to $C$. It turns out that the right solution is to rigidify (or normalize) the notion of $M$-polarization.

Definition 4.24. Let $S$ be a $K 3$ surface, and $j: M \hookrightarrow \operatorname{Pic}(S)$ a primitive lattice embedding. We say that $j$ is a normalized $M$-polarization if it satisfies the following two conditions:

(1) $j(h) \in \operatorname{Pic}(S)$ is the class of a nef divisor;

(2) for $i=1, \ldots, 5$ : if $\delta \in \Delta^{+}(S) \cap\left\langle j(h), \ldots, j\left(e_{i-1}\right)\right\rangle_{\operatorname{Pic}(S)}^{\perp}$, then $\delta . j\left(e_{i}\right) \leq 0$.

We now establish the main result of this section, the existence of an $M$-polarization in the non-generic case. The main idea is that we always have 5 intersection points, but some of them are "infinitely near points".

Proposition 4.25. Let $(C, L)$ be a degree 5 pair such that $C+L$ has at worst simple singularities and let $\widetilde{S}_{(C, L)}$ be the associated $K 3$ surface. Then $\widetilde{S}_{(C, L)}$ carries a normalized $M$-polarization $j: M \hookrightarrow \operatorname{Pic}\left(\widetilde{S}_{(C, L)}\right)$.

Proof. Let $S_{(C, L)}$ be the double cover of $\mathbb{P}^{2}$ branched along $C+L$. The surface $\widetilde{S}_{(C, L)}$ is obtained from $S_{(C, L)}$ by taking a minimal resolution of the singularities. In the case of double covers, there exists a standard procedure for obtaining the desingularization (e.g. [4, §III.7, p. 107]). Namely, there exists a commutative diagram:

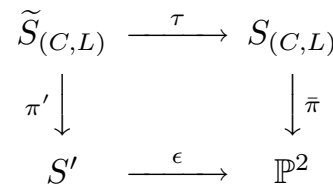

such that $\epsilon$ is a composition of blow-ups and $\pi^{\prime}$ is a double cover with smooth branch locus $B^{\prime} \subset S^{\prime}$. The surface $S^{\prime}$ is obtained by an inductive process. Start with $S_{0}=\mathbb{P}^{2}$ and $B_{0}=C+L$. Blow up a singular point of $B_{0}$, and let $\epsilon_{1}: S_{1} \rightarrow S_{0}$ be the resulting surface. The new branch divisor $B_{1}$ is the strict transform of $B_{0}$ together with the exceptional divisor of $\epsilon_{1}$ reduced mod 2. The process is repeated until the resulting divisor $B_{N}$ is smooth. Let $S^{\prime}=S_{N}, B^{\prime}=B_{N}$ and $\epsilon=\epsilon_{1} \ldots \epsilon_{N}$. The double cover of $S^{\prime}$ branched along $B^{\prime}$ is a minimal resolution of $S_{(C, L)}$.

Since the surface $\widetilde{S}_{(C, L)}$ does not depend on the order of the blow-ups, we choose to make the first 5 blow-ups in points belonging to (the strict transform of) the line $L$. By abuse of notation, we denote by $L$ the strict transform of the line $L$ on all the surfaces $S_{i}$ (including $S^{\prime}=S_{N}$ ). By construction, $L$ belongs to the branch divisor $B^{\prime}$. The inverse image $L^{\prime}$ of $L$ is a smooth rational curve on the $K 3$ surface $\widetilde{S}_{(C, L)}$. Thus, $\left(L^{\prime}\right)^{2}=-2$, which then gives $(L)^{2}=-4$. Since $L^{2}=1$ on $\mathbb{P}^{2}$ and $L$ is a smooth curve, it follows that $L$ is affected by exactly 5 blow-ups in 
the desingularization process. We choose these blow-ups as the first five steps of the sequence of blow-ups $S^{\prime} \rightarrow \cdots \rightarrow S_{1} \rightarrow \mathbb{P}^{2}$ (this is possible). Let $p_{i} \in L$ be centers of these blow-ups and $E_{i}$ the exceptional divisors (N.B. $p_{i} \in S_{i-1}$ and $E_{i}$ is a divisor on $S_{i}$ for $\left.i=1, \ldots, 5\right)$. We then define the following divisors:

$$
D_{i}=\pi^{\prime *} \epsilon_{N}^{*} \ldots \epsilon_{i+1}^{*}\left(E_{i}\right)
$$

for $i=1, \ldots, 5$.

The procedure described above produces 6 divisors $L^{\prime}, D_{1}, \ldots, D_{5}$ on the surface $\widetilde{S}_{(C, L)}$ such that the polarization class of $\widetilde{S}_{(C, L)}$ is

$$
H:=\left(\epsilon \circ \pi^{\prime}\right)^{*} L=2 L^{\prime}+D_{1}+\cdots+D_{5} .
$$

We immediately see that $L^{\prime}, D_{1}, \ldots, D_{5}$ satisfy the numerical conditions: $\left(L^{\prime}\right)^{2}=$ $-2, D_{i} . D_{j}=-2 \delta_{i j}$, and $L^{\prime} . D_{i}=1$. For example, since $E_{i}$ is an exceptional divisor we have $E_{i}^{2}=-1$ on $S_{i}$. It follows that the pullback of $E_{i}$ on $S^{\prime}$ will also have self-intersection -1 . Thus, on the double cover $\widetilde{S}_{(C, L)} \rightarrow S^{\prime}$ we get $D_{i}^{2}=-2$. In conclusion, the linear map $j: M \rightarrow \operatorname{Pic}\left(S_{(C, L)}\right)$ defined by sending $e_{i} \in M$ to be the class of $D_{i}$ given in (4.3) and $l^{\prime} \in M$ to the class of $L^{\prime}$ defines a lattice embedding. Since $j(h)$ corresponds to the polarization given by $H$, we obtain that $j$ is a primitive lattice embedding (cf. Remark 4.13).

The final aspect is to note that the embedding $j$ is normalized in the sense of Definition 4.24 (i.e. it satisfies the second requirement of the definition). This follows from the observation that the divisor $D_{i}$ on $\widetilde{S}_{(C, L)}$ is the fundamental cycle associated to the simple singularity of the curve $B_{i-1}$ in the point $p_{i}$.

Remark 4.26. We note that the $M$-polarization $j: M \hookrightarrow \operatorname{Pic}\left(\widetilde{S}_{(C, L)}\right)$ constructed in the previous proposition satisfies the following geometric properties:

i) $j(h)$ is the class of the base point free polarization $H$;

ii) $j\left(l^{\prime}\right)$ is the class of the irreducible rational curve $L^{\prime}$;

iii) $j\left(e_{1}\right), \ldots, j\left(e_{5}\right)$ are the classes of the effective divisors $D_{i}$.

We close by noting that the constructed $M$-polarizations for the surfaces $S_{(C, L)}$ fit well in families. Let $(\mathcal{C}, \mathcal{L}) \subset \mathbb{P}_{U}^{2} \rightarrow U$ be the universal family of degree 5 pairs satisfying the stability condition that $C+L$ has simple singularities. By taking a double cover, we obtain a family of surfaces $\mathcal{S} \rightarrow U$ with only du Val singularities and a flat family of rational curves $\mathcal{L}^{\prime} \rightarrow U$. After a finite base change, we can further assume that we have 5 sections $\sigma_{i}$ corresponding to the 5 points of intersection. By applying Brieskorn's simultaneous resolution to the family $\mathcal{S}$, we obtain a family of $K 3$ surfaces $\widetilde{\mathcal{S}} \rightarrow U$ (after a further finite base change of $U$ ). We note that the $M$-polarization in Proposition 4.25 is obtained by taking as the first 5 steps of the simultaneous resolution process the blow-up of $\mathcal{S}$ along the sections $\sigma_{i}$. In other words, the $M$-polarizations can be fitted together due to the fact that we can do the blow-up process of Proposition 4.25 in families (see the discussion of the simultaneous resolution from [16, pp. 128-135]). We get a family $\widetilde{\mathcal{S}} \rightarrow U$ of $M$-polarized $K 3$ surfaces, which gives a period map $\widetilde{\mathcal{P}}: U \rightarrow \mathcal{D} / O^{*}(T)$ (see [7]). This descends to a period map $\mathcal{P}: \mathcal{M} \rightarrow \mathcal{D} / \Gamma$ by noting that the construction of $M$-polarization depends only on the choice of a labeling of the intersection points $C \cap L$ (i.e. a surjective map $\sigma:\{1, \ldots, 5\} \rightarrow C \cap L$ respecting the intersection multiplicities). Passing from $\mathcal{D} / O^{*}(T)$ to $\mathcal{D} / \Gamma$ amounts to forgetting the labeling of the intersection (see the proof of Corollary 4.23). 
4.3. The proofs of Theorems 4.1 and 4.2, In 4.2 we have constructed a period map $\mathcal{P}: \mathcal{M} \rightarrow \mathcal{D} / \Gamma$ by sending a degree 5 pair $(C, L)$ to the periods of the $M$-polarized $K 3$ surface $\left(\widetilde{S}_{(C, L)}, j\right)$. Since $\mathcal{M}$ and $\mathcal{D} / \Gamma$ are normal quasi-projective varieties and the period map is algebraic, to prove Theorem 4.1 it suffices to prove that $\mathcal{P}$ is bijective. This amounts to showing that the data of $M$-polarization is rigid enough to recover uniquely the pair $(C, L)$ and, secondly, that any $M$ polarized $K 3$ surface is of type $\widetilde{S}_{(C, L)}$ for some degree 5 pair $(C, L)$. Once Theorem 4.1 is established, we obtain immediately the stronger statement, Theorem 4.2 by applying some general results of Looijenga 20] (see 44.3.3).

4.3.1. The surjectivity of the period map. The surjectivity of the period map $\mathcal{P}$ follows from the surjectivity of the period map for $K 3$ surfaces and Proposition 4.31 below. To prove Proposition 4.31, we need a series of technical lemmas. The first of those says that an $M$-polarized $K 3$ surface is a double cover of $\mathbb{P}^{2}$.

Lemma 4.27. Let $(S, j)$ be an $M$-polarized $K 3$ surface such that $j(h)$ is nef and $j\left(e_{1}\right), \ldots, j\left(e_{5}\right)$ are classes of effective divisors. Then, the complete linear system defined by $j(h)$ is base point free.

Proof. By assumption, we can represent the classes $j\left(l^{\prime}\right), j\left(\delta_{1}\right), \ldots, j\left(\delta_{5}\right)$ by effective divisors $L^{\prime}, D_{1}, \ldots, D_{5}$. Let $H=2 L^{\prime}+D_{1}+\cdots+D_{5}$. Assuming that $|H|$ is not base point free, we get $H \equiv 2 E+R$ for some smooth elliptic curve $E$ with $H$.E $=1$ (cf. Theorem 4.8). In particular, note that $E$ is nef. Since $L^{\prime}, D_{1}, \ldots, D_{5}$ are effective, it follows from $H . E=1$ that we can assume E. $L^{\prime}=\cdots=E \cdot D_{4}=0$ and E. $D_{5}=1$. Let $F_{5}=H-E_{5}$ and $D=2 E-F_{5}$ with classes $j\left(f_{5}\right)$ and $d$ respectively. We then have D. $L^{\prime}=\cdots=D \cdot D_{5}=0$ and $D^{2}=0$, which gives $d \in M_{\operatorname{Pic}(S)}^{\perp}$ and $d^{2}=0$. Since both $M$ and $\operatorname{Pic}(S)$ are hyperbolic, the lattice $M_{\operatorname{Pic}(S)}^{\perp}$ is negative definite. In conclusion, we obtain $d=0$. Thus, we have $j\left(f_{5}\right)=2 e$, where $e \in \operatorname{Pic}(S)$ is the class of the curve $E$. There are two possibilities: either $e \in j(M)$ or $e \notin j(M)$. The former case is not possible, since $f_{5}$ is not divisible in $M$ (see the proof of Proposition 4.14). The latter implies that the embedding $j: M \hookrightarrow \operatorname{Pic}(S)$ is not primitive (see the proof of Proposition 4.12), but this contradicts the assumptions. Therefore, the complete linear system $|H|$ is base point free.

The following lemma shows that any $M$-polarization can be normalized.

Lemma 4.28. Let $(S, j)$ be an $M$-polarized $K 3$ surface. Then there exists a $\phi \in$ $\pm W(S)$ such that the composite map

$$
M \stackrel{j}{\rightarrow} \operatorname{Pic}(S) \stackrel{\phi}{\rightarrow} \operatorname{Pic}(S)
$$

defines a normalized $M$-polarization for $S$.

Proof. By acting with \pm 1 , we can assume that $j(h) \in V^{+}(S)$. It is known that

$$
C(S)=\left\{x \in V^{+}(S) \mid x . \delta \geq 0 \text { for all } \delta \in \Delta^{+}(S)\right\}
$$

is a fundamental domain for the action of $W(S)$ on $V^{+}(S)$ (e.g. [4 p. 313]). Thus, there exists a $\phi \in W(S)$ such that changing the embedding by $\phi$, we have $\phi(j(h))$ is nef. It follows that we can assume $j(h)$ is nef. Acting with reflection in roots orthogonal to $j(h)$ preserves this condition.

Let $R$ be the sublattice of $\langle j(h)\rangle_{\operatorname{Pic}(S)}^{\perp}$ spanned by the roots. We have $j\left(e_{i}\right) \in R$ and $R$ is an even negative definite root lattice. In particular, $R$ decomposes as a 
direct sum $R_{1} \oplus \cdots \oplus R_{k}$ of irreducible root systems of type $A-D-E$. We can assume that $j\left(e_{1}\right) \in R_{1}$. By acting with $W\left(R_{1}\right)$, we can arrange that $j\left(e_{1}\right)$ is the highest root of $R_{1}$ (N.B. $\Delta^{+}(S)$ determines the set of positive roots for $R_{1}$ ). Since $j(h)$ is fixed by $W\left(R_{1}\right), j(h)$ remains nef. By construction, $j\left(e_{1}\right)$ satisfies the second condition of Definition 4.24 , i.e. $\delta \in\langle h\rangle_{\operatorname{Pic}(S)}^{\perp} \cap \Delta^{+}(S) \Longrightarrow \delta . j\left(e_{1}\right) \leq 0$. The claim follows by repeating the process for $j\left(e_{i}\right)$ (for $i=2, \ldots, 5$ ), but acting only with reflections that stabilize $j(h), \ldots, j\left(e_{i-1}\right)$.

Note that a normalized $M$-polarization is essentially unique.

Lemma 4.29. Assume that $S$ is a $K 3$ surface with two normalized $M$-polarizations $j$ and $j^{\prime}$. Assume that $j(h)=j\left(h^{\prime}\right)$ and $j\left(l^{\prime}\right)=j^{\prime}\left(l^{\prime}\right)$. Then up to a permutation of the labeling of $e_{i}$, we have $j=j^{\prime}$ (i.e. $j\left(e_{i}\right)=j^{\prime}\left(e_{\sigma(i)}\right)$ for a permutation $\left.\sigma\right)$.

Proof. Note first that for root $\delta \in \operatorname{Pic}(S)$ such that $\delta . j^{\prime}(h) \neq 0$ and $j^{\prime}(h) \not \perp j^{\prime}(M)$ we must have $\delta \cdot j^{\prime}\left(e_{i}\right) \neq 0$ for some $i$. By the definition of normalized polarization, $j\left(e_{1}\right)$ is the highest root of an irreducible summand in $\langle j(h)\rangle_{\operatorname{Pic}(S)}^{\perp}$ (w.r.t. $\Delta^{+}(S)$ ). Since $j\left(l^{\prime}\right)=j^{\prime}\left(l^{\prime}\right)$ and $j\left(l^{\prime}\right) \cdot j\left(e_{1}\right)=1$, we conclude that $j\left(e_{1}\right) \cdot j^{\prime}\left(e_{i}\right) \neq 0$ for some $i$. Thus, $j\left(e_{1}\right)$ and $j^{\prime}\left(e_{i}\right)$ belong to the same irreducible root system in $\langle j(h)\rangle_{\operatorname{Pic}(S)}^{\perp}=$ $\left\langle j^{\prime}(h)\right\rangle_{\mathrm{Pic}(S)}^{\perp}$. Since $j\left(e_{1}\right)$ is the highest root, from the fact that $j^{\prime}$ is also normalized we conclude that $j\left(e_{1}\right)=j^{\prime}\left(e_{k}\right)$ for some $k \leq i$. We can assume $k=1$, and the argument can be repeated for the remaining $e_{i}$.

The last preliminary result shows that a normalized $M$-polarized $K 3$ surface $(S, j)$ satisfies the geometric properties listed in Remark 4.26 (esp. ii). This is an important fact that allows us to pass from an $M$-polarization to a degree 5 pair. We note that the basic idea of the proof of the lemma is that the condition of normalized polarization forces all the (-2)-curves orthogonal to $j(h)$, but not orthogonal to $j(M)$, to be components of $j\left(e_{1}\right), \ldots, j\left(e_{5}\right)$. It follows then that $j\left(l^{\prime}\right)$ is irreducible.

Lemma 4.30. Assume that $(S, j)$ is a normalized $M$-polarization. Then $j\left(l^{\prime}\right)$ is the class of an irreducible curve (thus, smooth and rational).

Proof. As before, we represent the classes $j\left(l^{\prime}\right), j(h), \ldots, j\left(e_{5}\right)$ by effective divisors $H, L^{\prime}, D_{1}, \ldots, D_{5}$. By Lemma 4.27, we get that $H$ is a base point free polarization. Assuming that $L^{\prime}$ is not irreducible, we have a decomposition:

$$
L^{\prime}=\sum n_{i} C_{i}+\sum m_{i} R_{i},
$$

where $n_{i}, m_{i} \geq 0$, and $C_{i}$ and $R_{i}$ are irreducible curves with $C_{i}^{2} \geq 0$ and $R_{i}^{2}=-2$.

We note first that there cannot be any non-rational curve occurring in (4.4). From the Hodge index theorem, we have $C_{i}^{2} \cdot H^{2} \leq\left(H . C_{i}\right)^{2}$ with equality only if $C_{i}$ and $H$ are proportional. If $C_{i}^{2}>0$, we get $H . L^{\prime} \geq H . C_{i} \geq 2$, contradicting the assumption $H \cdot L^{\prime}=1$. Similarly, if $C_{i}^{2}=0$ we get $C_{i} . H=1$, a contradiction to $|H|$ being base point free (see Proposition 4.9). It follows that $L^{\prime}$ has the following decomposition:

$$
L^{\prime}=R_{0}+\sum_{i=1}^{k} m_{i} R_{i}
$$


where $R_{0}, \ldots, R_{k}$ are irreducible rational curves such that $H \cdot R_{0}=1$ and $H \cdot R_{i}=0$ for $i=1, \ldots, k$. Since $R_{0}^{2}=L^{\prime 2}=-2$, we get

$$
R_{0} \cdot\left(\sum m_{i} R_{i}\right)=-\frac{1}{2}\left(\sum m_{i} R_{i}\right)^{2} \geq 1 .
$$

Thus, $R_{0} \cdot L^{\prime} \geq-1$. On the other hand, it is easy to see that $R_{0} \cdot D_{i} \geq 0$. We conclude $1=R_{0} . H \geq 2 R_{0} . L^{\prime}$, which together with (4.6) gives $R_{0} \cdot L^{\prime} \in\{0,-1\}$.

Assume that $R_{0} \cdot L^{\prime}=0$. Using $H \cdot R_{0}=1$, we get $D_{i} . R_{0}=1$ for exactly one value of $i$, and $D_{i} \cdot R_{0}=0$ for the remaining values. Assume first that $D_{1} \cdot R_{0}=0$. Since $D_{1} \cdot L^{\prime}=1$, we get $D_{1} \cdot\left(\sum m_{i} R_{i}\right)=1$. By assumption the embedding $j$ is normalized. In particular, $D_{1}$ is the highest root in $\langle j(h)\rangle^{\perp}$. Since the $R_{i}$ are effective roots in $\langle h\rangle^{\perp}$, we get $D_{1} . R_{i} \leq 0$ for all $i=1, \ldots, k$. Thus, $1=$ $D_{1}$. $\left(\sum m_{i} R_{i}\right) \leq 0$, a contradiction. It follows that we must have $D_{1} \cdot R_{0}=1$. The same argument as above gives $D_{1} \cdot R_{i}=0$ for all $i$. Then the $R_{i}$ are effective roots in $\left\langle j(h), j\left(e_{1}\right)\right\rangle^{\perp}$ and we obtain a contradiction to $D_{2} \cdot R_{0}=0$.

The case $R_{0} \cdot L^{\prime}=-1$ is handled by a similar computation. We omit the details. In conclusion, the decomposition (4.5) is trivial, i.e. $L^{\prime}$ is irreducible.

We can now conclude that any $M$-polarized $K 3$ surface is of type $\widetilde{S}_{(C, L)}$.

Proposition 4.31. Let $S$ be an $M$-polarized $K 3$ surface. Then, there exists a degree 5 pair $(C, L)$ such that $S \cong \widetilde{S}_{(C, L)}$.

Proof. We are given a $K 3$ surface $S$ together with a primitive lattice embedding $j: M \hookrightarrow \operatorname{Pic}(S)$. Without loss of generality, we can assume that $j$ is a normalized embedding (cf. Lemma 4.28). In particular, $j(h)$ is nef and $j\left(e_{i}\right)$ are classes of effective divisors. It follows that $j(h)$ is base point free (cf. Lemma 4.27). Thus, $j(h)$ defines a generically $2: 1$ morphism $\pi: S \rightarrow \mathbb{P}^{2}$, with branch curve a sextic $B$.

By Lemma 4.30, the class $j\left(l^{\prime}\right)$ contains a (unique) smooth rational curve $L^{\prime}$. It follows that $L=\pi_{*} L^{\prime}$ is a line in $\mathbb{P}^{2}$. To prove that $L$ is a component of the branch locus $B$, it is enough to prove that class $j\left(l^{\prime}\right)$ of $L^{\prime}$ is invariant under involution $i$, where $\tau$ is the natural sheet-exchanging involution on $S$ and $i=\tau^{*}$ the induced involution on $H^{2}(S, \mathbb{Z})$. The polarization class $j(h)$ is left invariant by $i$. We claim that also the classes $j\left(e_{i}\right)$ are invariant. Namely, the morphism $\pi$ will contract all the $(-2)$-curves orthogonal to $j(h)$ to singularities of the sextic $B$. From the normalization assumption, it follows that $j\left(e_{1}\right)$ is the fundamental cycle of a singularity of $B$. Thus, $j\left(e_{1}\right)$ is invariant. A similar argument works for all $e_{i}$. Since $2 l^{\prime}=h-e_{1}-\cdots-e_{5}$, we conclude that $j\left(l^{\prime}\right)$ is also invariant as needed.

4.3.2. The proof of Theorem 4.1. First, the surjectivity follows from Proposition 4.31, which assures us that the construction of $\$ 4.2$ can be reversed. Given a point $w \in \mathcal{D} / \Gamma$, we choose a lift $\omega \in T_{\mathbb{C}} \subset \Lambda_{\mathbb{C}}$. This determines a lattice $P:=\Lambda \cap\langle\omega\rangle^{\perp}$ and a factorization of the fixed primitive embedding $M \hookrightarrow \Lambda$ into $M \hookrightarrow P \hookrightarrow \Lambda$. To associate a $K 3$ surface $S$ to $\omega$, we have to provide a choice for $V^{+}(P)$ and $\Delta^{+}(P) \subset \Delta(P)$. We define $V^{+}(P)$ to be the connected component of $V(P)$ that contains $j(h)$. Then, we define the partition $\Delta(P)=\Delta^{+}(P) \sqcup\left(-\Delta^{+}(P)\right)$ by:

i) if $\delta \in \Delta(P)$ and $\delta . j(h) \neq 0: \delta \in \Delta^{+}(S)$ if $\delta . j(h)>0$ or $-\delta \in \Delta^{+}(P)$ otherwise;

ii) if $\delta \in\left\langle j(h), \ldots, j\left(\delta_{i-1}\right)\right\rangle \frac{\perp}{P} \cap \Delta(P)$ and $\delta . j\left(\delta_{i}\right) \neq 0: \delta \in \Delta^{+}(P)$ if $\delta . j\left(\delta_{i}\right)<0$ or $-\delta \in \Delta^{+}(P)$ otherwise (for $i=1, \ldots, 5$ and $\delta_{0}=h$ );

iii) for $\Delta(P) \cap j(M) \stackrel{\perp}{P}$ we choose an arbitrary Weyl chamber. 
By the surjectivity of the period map for $K 3$ surfaces, there exists a $K 3$ surface $S$ with period point $\omega$ and such that $\operatorname{Pic}(S)=P, V^{+}(S)=V^{+}(P)$, and $\Delta^{+}(S)=$ $\Delta^{+}(P)$. By construction $j: M \hookrightarrow \operatorname{Pic}(S)$ is a normalized $M$-polarization. Thus, there exists a degree 5 pair $(C, L)$ such that $S \cong \widetilde{S}_{(C, L)}$ (cf. Proposition 4.31).

By the global Torelli theorem, the surface $S$ obtained above is unique up to isomorphism. The lattice $M$ is left invariant by $O^{*}(T)$. It is easily seen that the classes $h$ and $l^{\prime}$ are left invariant by the full monodromy group $\Gamma=O_{-}(T)$ (see Proposition 4.22). It follows that the period point $\omega \in \mathcal{D} / \Gamma$ uniquely determines the classes $j(h), j\left(l^{\prime}\right) \in \operatorname{Pic}(S)$. The polarization $j(h)$ determines the double cover map $S \rightarrow \mathbb{P}^{2}$ with branch curve $B$, and $j\left(l^{\prime}\right)$ determines a line component $L$ of $B$. Thus, $B=C+L$, and we conclude that a period point $\omega$ determines uniquely (up to projective isomorphism) a degree 5 pair $(C, L)$.

4.3.3. The proof of Theorem 4.2. E. Looijenga 20] has developed a general framework of comparing GIT compactifications to appropriate compactifications of the period space. Specifically, [20, Thm. 7.6] says that once a period map gives an isomorphism $\mathcal{M} \cong(\mathcal{D} \backslash \mathcal{H}) / \Gamma$ between a geometric quotient $\mathcal{M}$ and the complement of an arithmetic arrangement $\mathcal{H}$ of hyperplanes in a type IV domain, it automatically extends to an isomorphism $\overline{\mathcal{M}} \cong \widetilde{\mathcal{D} / \Gamma}$ between the GIT compactification of $\mathcal{M}$ and the Looijenga compactification associated to $\mathcal{H}$.

In our situation, we apply [20, Thm. 7.6] to the empty arrangement of hyperplanes. By Theorem 4.1, we have an isomorphism $\mathcal{M} \cong \mathcal{D} / \Gamma$. Since the Looijenga compactification associated to the empty arrangement of hyperplanes is precisely the Baily-Borel compactification $(\mathcal{D} / \Gamma)^{*}$, we conclude that Theorem 4.2 holds once the technical assumptions of [20, Thm. 7.6] are verified. In our situation, this is easy. First, the codimension condition for the GIT quotient is clearly satisfied. The complement of the $G$-invariant open subset $U$ parameterizing degree 5 pairs $(C, L)$ such that $C+L$ has simple singularities has high codimension in $X^{s s}(1)$. The second assumption in [20, Thm. 7.6] is that the isomorphism $\mathcal{M} \cong \mathcal{D} / \Gamma$ is an isomorphism of polarized varieties (both spaces are naturally polarized). Since $\mathcal{M}$ is an open subset of the GIT quotient $\mathcal{M}(1)$, its polarization is obtained by restricting the polarization of the moduli space of plane sextics (see Theorem 2.4). Similarly, the polarization on $\mathcal{D} / \Gamma$ is obtained by restricting the polarization of the moduli space of degree two $K 3$ surfaces. Thus the identification of polarizations on $\mathcal{M}$ and $\mathcal{D} / \Gamma$ follows from Looijenga's computation [20, §8] (esp. [20, Thm. 8.6]) for degree two $K 3$ surfaces.

4.4. The Baily-Borel compactification. The quotient of a bounded symmetric domain by an arithmetic group $\mathcal{D} / \Gamma$ admits a canonical minimal compactification, the Baily-Borel compactification $(\mathcal{D} / \Gamma)^{*}$. In the case of Type IV domains, the boundary components of $(\mathcal{D} / \Gamma)^{*}$ are either 0-dimensional (type III) or 1-dimensional (type II), and are in bijective correspondence with the equivalence classes of the primitive isotropic sublattices of $T$ of ranks 1 and 2 respectively. Thus, to determine the Baily-Borel compactification is a pure arithmetic question, which in our situation has the following answer:

Theorem 4.32. The boundary of $\mathcal{D} / \Gamma$ in the Baily-Borel compactification $(\mathcal{D} / \Gamma)^{*}$ consists of two 0-dimensional components and four 1-dimensional components. Their incidence graph is given in Figure 4 , 
Proof. The 0-dimensional boundary components correspond to the classes of rank 1 isotropic lattices of $T$. These are classified in 4.4.1 (esp. Lemma 4.35). Lemma 4.36 reduces the classification of isotropic rank 2 lattices to the classification of isotropic vectors in the hyperbolic lattice $N:=E_{8} \oplus D_{4} \oplus U(2)$. We conclude by Vinberg's algorithm applied to $N$ (see $\$ 4.4 .2$ ).

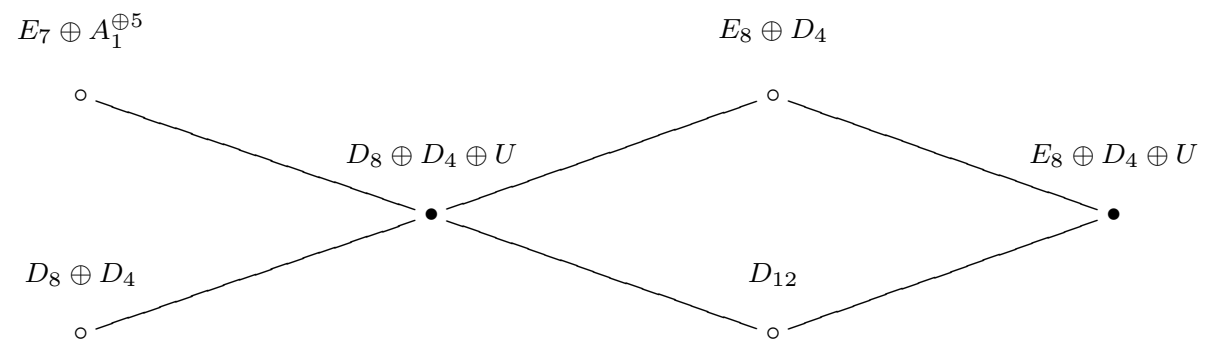

FIgURE 4. The boundary components of the Baily-Borel compactification

According to Theorem 4.2, the projective varieties $\mathcal{M}(1)$ and $(\mathcal{D} / \Gamma)^{*}$ are isomorphic. Since they compactify the same space $\mathcal{M} \cong \mathcal{D} / \Gamma$, the two sets of boundary components are isomorphic. Geometrically, the matching of the boundary components (Figures 2 and (4) is obtained as follows. Let $\left(C_{0}, L_{0}\right)$ be a degree 5 pair corresponding to a boundary point of $\mathcal{M}(1) \backslash \mathcal{M}$. We consider a generic family of degree 5 pairs $\left(C_{t}, L_{t}\right)$ degenerating to $\left(C_{0}, L_{0}\right)$. This produces a family of $K 3$ surfaces $S_{t}$ (where $S_{t}=\widetilde{S}_{\left(C_{t}, L_{t}\right)}$ ) degenerating to $S_{0}$, the double cover corresponding to $\left(C_{0}, L_{0}\right)$. To the family of $K 3$ surfaces $\left(S_{t}\right)_{t \in \Delta^{*}}$ there is associated a canonical limit mixed Hodge structure $H_{\text {lim }}^{2}$, which determines a boundary point in the Baily-Borel compactification. Since the period map extends to the boundary, the resulting point does not depend on the choice of the degenerating family. Thus, to compute the limit mixed Hodge structure $H_{\mathrm{lim}}^{2}$ it is enough to do semistable reduction and apply the Clemens-Schmid sequence (e.g. 22]) for a suitable degeneration to $\left(C_{0}, L_{0}\right)$. One distinguishes two cases, types II and III, depending on the monodromy of the family. Using the incidence relation of the boundary components, the matching for the type III case is obtained from that for type II. Finally, the computation in the type II case is very similar to that for degree two $K 3$ surfaces (e.g. [10, Rem. 5.6]). We conclude:

Corollary 4.33. Via the extended period map $\overline{\mathcal{P}}: \mathcal{M}(1) \rightarrow(\mathcal{D} / \Gamma)^{*}$, the boundary components of the GIT quotient $\mathcal{M}(1)$ map to boundary components of the BailyBorel compactification $(\mathcal{D} / \Gamma)^{*}$ as given in Table 5 .

4.4.1. Invariants of isotropic sublattices. Explicit computations of the Baily-Borel compactification for type IV domains are given in [6, 33, 36. The computations in our situation are similar, and we only sketch the arguments.

To compute the Baily-Borel compactification of the period space $\mathcal{D} / \Gamma$ we have to classify the isomorphism classes of isotropic sublattices $E$ of the transcendental 
TABLE 5. The matching of the boundary components

\begin{tabular}{ll}
\hline GIT boundary (see Figure 2]) & Baily-Borel boundary (see Figure [4) \\
\hline II(1) & $E_{8} \oplus D_{4}$ \\
II(2a) & $E_{7} \oplus A_{1}^{\oplus 5}$ \\
II(2b) & $D_{4} \oplus D_{8}$ \\
II(3) & $D_{12}$ \\
\hline III(1) & $E_{8} \oplus D_{4} \oplus U$ \\
III $(2)$ & $D_{8} \oplus D_{4} \oplus U$ \\
\hline
\end{tabular}

lattice $T \cong D_{4} \oplus E_{8} \oplus U(2) \oplus U$ modulo $O(T)$ (N.B. $\Gamma=O_{-}(T)$ and $\mathbb{Z} / 2 \mathbb{Z} \cong$ $O(T) / O_{-}(T)$ correspond to the choice of component for $\left.\mathcal{D}\right)$. We start by noting the following invariants for an isotropic lattice $E$ (see 6, 33):

- the rank $k \in\{1,2\}$ of $E$;

- the isotropic subgroup $H_{E}:=E_{T^{*}}^{\perp} / E$ of the discriminant group $A_{T}$;

- the isomorphism class of the lattice $E^{\perp} / E$.

The main conclusion of our computations below is that these invariants completely determine the isomorphism classes of the isotropic sublattices of $T$.

For the moment, let us describe the possible values for these invariants. Since the discriminant group $A_{T}$ is isomorphic to $(\mathbb{Z} / 2 \mathbb{Z})^{4}$, it follows easily that $H_{E}$ is either trivial or isomorphic to $\mathbb{Z} / 2 \mathbb{Z}$. Furthermore, in the latter case, all possible subgroups $H_{E}$ are conjugate by $O(T)$. Fixing the rank $k$ and the group $H_{E}$ is equivalent to specifying the signature and the discriminant group for the lattice $E^{\perp} / E$ (N.B. $\left.A_{E^{\perp} / E} \cong H_{E}^{\perp} / H_{E}\right)$. In particular, since the lattice $E^{\perp} / E$ is 2-elementary, it follows that $k$ and $H_{E}$ determine the genus of $E^{\perp} / E$ ([27, Thm. 3.6.2]). In the rank 1 case, the lattice $E^{\perp} / E$ is indefinite and its isomorphism class is completely determined by the genus. Thus, if $E$ is isotropic of rank 1, then $E^{\perp} / E$ is isomorphic to either $D_{4} \oplus D_{8} \oplus U$ or $D_{4} \oplus E_{8} \oplus U$. In the rank 2 case, the two possibilities for $H_{E}$ determine two genera for $E^{\perp} / E$, namely $\mathcal{G}\left(D_{4} \oplus D_{8}\right)$ and $\mathcal{G}\left(D_{4} \oplus E_{8}\right)$, which in turn give 4 possibilities for the isomorphism classes.

Lemma 4.34. The genera of $\mathcal{G}\left(D_{4} \oplus D_{8}\right)$ and $\mathcal{G}\left(D_{4} \oplus E_{8}\right)$ contain exactly two isomorphism classes of even negative definite lattices, namely

i) $\mathcal{G}\left(D_{4} \oplus E_{8}\right)$ consists of the root lattices $D_{4} \oplus E_{8}$ and $D_{12}$;

ii) $\mathcal{G}\left(D_{4} \oplus D_{8}\right)$ consists of the lattice $D_{4} \oplus D_{8}$ and an index 2 overlattice of $E_{7} \oplus A_{1}^{5}$.

Proof. By [33, Prop. 6.1.1], every lattice in the genus of $L=D_{4} \oplus E_{8}$ can be obtained as the orthogonal complement $L_{\Lambda}^{\perp}$ for a suitable primitive embedding of $L$ into an even unimodular negative definite lattice $\Lambda$ of rank 24. Since $L$ is a root lattice, the embedding $L \hookrightarrow \Lambda$ factors as $L \hookrightarrow \underline{\Lambda} \subseteq \Lambda$, where $\underline{\Lambda}$ is the sublattice spanned by the roots of $\Lambda$. The possibilities for $\Lambda$ (and $\underline{\Lambda}$ ) are classified by Niemer's theorem. Finally, since the embeddings of root lattices are well understood, we conclude by a case-by-case analysis. The item ii is similar.

It is easy to see that each choice of admissible invariants actually corresponds to some isotropic sublattice $E$. For example, there exists a rank 2 isotropic sublattice $E$ such that $E^{\perp} / E \cong D_{12}$ by the following argument. Since the lattice $T$ is determined by its invariants, there exists an isomorphism $T \cong D_{12} \oplus U \oplus U(2)$. 
By choosing an isotropic vector in each of the hyperbolic summands, we obtain a rank 2 isotropic sublattice $E$ having the right invariants. In conclusion, we have obtained 6 boundary components for the Baily-Borel compactification satisfying the incidence relations of Figure 4 (N.B. the label in Figure 4 corresponds to the isomorphism class of $E^{\perp} / E$ ). The remaining part for the proof of Theorem 4.32 is to see the converse. There exists at most one class of isotropic sublattices having some prescribed invariants. For the rank 1 case, this is automatic by the following result of Brieskorn [6, Kor. 2, p. 87].

Lemma 4.35. Let $T$ be an even lattice containing at least two hyperbolic planes such that the natural map $O(T) \rightarrow O\left(q_{T}\right)$ is surjective. Then the classes of rank 1 isotropic sublattices modulo $O(T)$ are in bijection with the classes of isotropic vectors in $A_{T}$ modulo $\pm O\left(q_{T}\right)$.

In our situation, $T \cong D_{4} \oplus D_{8} \oplus U^{2}$ and $O(T) \rightarrow O\left(q_{T}\right)$ is surjective; thus we can apply the previous lemma. The bijection of the lemma is given by associating to an isotropic lattice $E$ the generator of $H_{E}$. Thus, we obtain indeed only two classes of isotropic rank 1 sublattices (distinguished by the invariant $H_{E}$ ).

4.4.2. Rank 2 isotropic sublattices. Let $N \cong E_{8} \oplus D_{4} \oplus U(2)$ be the sublattice of $T$ obtained by splitting off a hyperbolic plane from $T$. We claim that the classification of the isotropic rank 2 sublattices of $T \cong N \oplus U$ is essentially equivalent to the classification of the isotropic rank 1 sublattices in $N$. The idea is simple. Namely, given $E$ a rank 2 isotropic sublattice we can choose a rank 1 sublattice $E^{\prime}$ in $E$ and use the classification of $E^{\prime}$ given by Lemma 4.35 to reduce to $N$. More precisely, we have:

Lemma 4.36. Let $E$ be a rank 2 isotropic sublattice of $T$. Then there exists a basis $\left\{b_{1}, b_{2}\right\}$ of $E$ and an isometry $\phi: T \rightarrow U \oplus N$ such that $\phi\left(b_{1}\right) \in U$ and $\phi\left(b_{2}\right) \in N$.

Thus, to classify $E$ it is enough to classify the isotropic rank 1 sublattices in $N$ modulo $O(N)$. Note that going back from an isotropic vector in $N$ to an isotropic rank 2 sublattice in $T$ might give some repetitions, but these are easy to detect. In conclusion, we are done once we classify the isotropic vectors in $N$.

Since $N$ contains only one hyperbolic plane, Lemma 4.35 cannot be applied. Instead, we use the fact that $N$ is a hyperbolic lattice, i.e. it has signature $(1, n)=$ $(1,13)$. For such lattices there exists a classification algorithm for the isotropic vectors due to Vinberg [39, §1.4] (see also [36, §4.3]). The algorithm starts by fixing an element $h \in N$ of positive square. Then, the algorithm consists of inductively choosing roots $\delta_{1}, \delta_{2}, \ldots$ such that a certain distance function to $h$ is minimized. The algorithm stops with the choice of the root $\delta_{N}$ if the following stop condition ([39. Thm. 2.6bis]) is satisfied: every connected parabolic subdiagram of the Dynkin diagram $\Sigma$ associated to the roots $\delta_{1}, \ldots, \delta_{N}$ is a connected component of some parabolic subdiagram of rank $n-1$ (i.e. the maximal rank). If the algorithm stops, the classes of isotropic vectors in $N$ are determined by the rule: every isotropic line $E$ in $N$ is equivalent to the null space of the lattice corresponding to a parabolic subdiagram of rank $n-1$ of the Dynkin diagram $\Sigma$. We recall that by a parabolic diagram we understand the extended Dynkin diagram of a root system, and the rank is the rank of the corresponding root system.

In our situation, a straightforward application of Vinberg's algorithm for $N$ produces the Dynkin diagram $\Sigma$ given in Figure 5. The computation is simplified by 


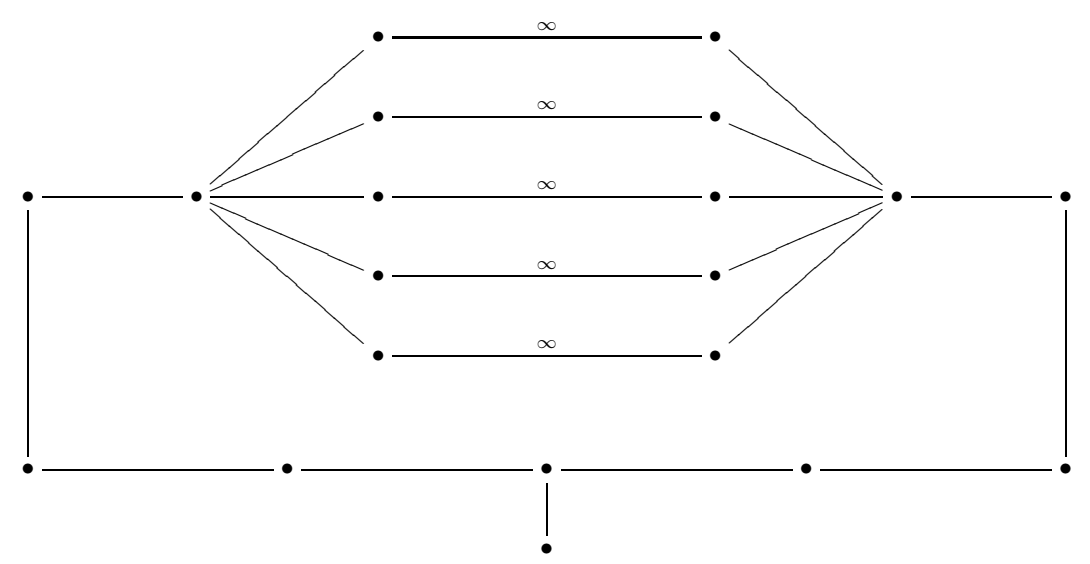

FiguRE 5. Vinberg diagram for $N$

the observation $N \cong M \oplus E$. This gives the natural choice $h \in M$ (the polarization class used in $(4.2)$ to start the algorithm. Note also that the Dynkin diagram $\Sigma$ satisfies the stopping criterion. Up to the obvious symmetries, there are only 4 distinct possibilities for the maximal rank parabolic subdiagrams of the Dynkin diagram of Figure 5, namely $\widetilde{A}_{1}^{\oplus 5} \oplus \widetilde{E}_{7}, \widetilde{D}_{8} \oplus \widetilde{D}_{4}, \widetilde{E}_{8} \oplus \widetilde{D}_{4}$, and $\widetilde{D}_{12}$. Together with Lemma 4.34, this implies that there are exactly 4 classes of isotropic rank 2 sublattices of $T$ modulo $O(T)$. This concludes the proof of Theorem 4.32 .

\section{Applications to the deformations of $N_{16}$}

In this section, we return to our original motivation, the study of the deformations of $N_{16}$. We consider the germ $\left(Y_{0}, y_{0}\right)$ of a singularity of type $N_{16}$ and let $\mathcal{Y} \rightarrow S$ be the semi-universal deformation. Since $Y_{0}$ is a hypersurface singularity, the base $S$ is the germ of a smooth 16-dimensional space $\left(\mu=\tau=16\right.$ for $\left.N_{16}\right)$. The main question that one would like to understand is the natural stratification of $S$ in terms of the singularities of the nearby fibers $Y_{s}$. We recall that there exists an open dense stratum of $S$ which parameterizes the smooth fibers $Y_{s}$, whose complement is called the discriminant hypersurface $\Sigma \subset S$. The discriminant $\Sigma$ is stratified in terms of the complexity of the singularities occurring for $Y_{s}(s \in \Sigma)$. For instance, we can discuss the equisingular stratum, the stratum of simple singularities, the stratum of simple elliptic singularities, etc. The basic question, then, is to say something about the structure of this stratification. The easiest and the best understood situation is that of the deformations of the simple singularities. In that case, $S$ can be taken to be the quotient of the affine space $\mathbb{A}^{\mu}$ by a finite group $W$, and the discriminant corresponds to $\mathcal{H} / W$, where $\mathcal{H}$ is an arrangement of hyperplanes in $\mathbb{A}^{\mu}$. Furthermore, the stratification of the discriminant corresponds to the natural stratification given by the intersections of hyperplanes from $\mathcal{H}$ (e.g. [1, Ch. 5]). By work of Pinkham [30, 29, Looijenga [18, 19], Brieskorn [5], and others, a rather similar situation was shown to hold also for the unimodal (e.g. triangle) singularities. In our situation, putting together the results of the previous sections, we show that the structure of the deformations of $N_{16}$ is very similar to the structure of the deformations of the triangle singularities. The only 
major difference is that one has to work in a relative setting over the equisingular deformations.

In our study of the deformations of $N_{16}$, we make the following two standard restrictions (see [19], [20, §10]). First, we consider only the case of quasi-homogeneous $N_{16}$. This restriction implies the existence of a natural $\mathbb{C}^{*}$-action on the base $S$ of the versal deformation, which in turn determines subspaces $S_{\leq 0}, S_{0}, S_{+}$, etc. (see \$2.4). In our situation, the space of the deformations of positive weight $S_{+}$ (the equisingular deformations moving away from the $\mathbb{C}^{*}$-action) is 1-dimensional. The second restriction is to ignore these deformations; i.e. we consider only the codimension 1 subspace $S_{\leq 0}$ of $S$. This type of restriction is harmless, at least from a topological point of view. By results of Wirthmüller and Damon, for nonsimple hypersurface germs, the universal deformation is topologically trivial along the Hessian direction (i.e. the positive weight direction in our situation).

Due to the assumptions made in the previous paragraph, we are in the situation covered by the theory of deformations with $\mathbb{C}^{*}$-action of Pinkham 28. It follows that the non-positive weight deformations of $N_{16}$ can be identified with the projective deformations of the pair $\left(C_{0}, L_{0}\right)$, where $C_{0} \subset \mathbb{P}^{2}$ is the cone over 5 points in $\mathbb{P}^{1}$ and $L_{0}$ is a generic line. By gluing together these deformations in a stack, we obtain a modular interpretation for the deformations of non-positive weight of $N_{16}$. As discussed in section 2 (esp. $\$ 2.4$ ), we obtain a diagram:

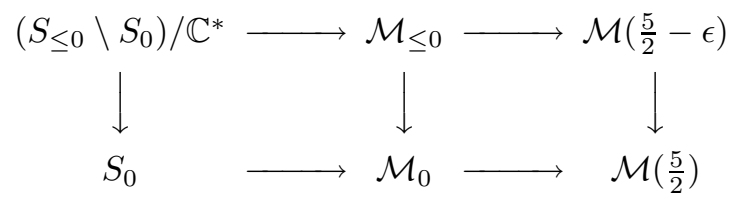

where $\mathcal{M}_{0}$ is the moduli of 5 distinct points in $\mathbb{P}^{1}, \mathcal{M}_{\leq 0}$ is the moduli space of pairs $(C, L)$ such that $C$ is not a cone and $C \cap L$ is a transversal, and the map $\mathcal{M}_{\leq 0} \rightarrow \mathcal{M}_{0}$ is the natural forgetful map sending $(C, L)$ to the intersection $C \cap L$. The left side square of (5.1) is the usual process of passing from deformations to the (coarse) moduli space, and the right side square is the compactification given by the variation of GIT quotients. Note that everything is done in a relative version over the weight 0 deformations (the equisingular deformations preserving the $\mathbb{C}^{*}$ action). By restricting to fibers, we obtain the deformations of negative weight (the smoothing directions) and the usual globalization of Pinkham. For comparison, we note that for triangle singularities, $S_{0}$ is trivial (i.e. a point).

As mentioned above, we are concerned here with the natural stratification of $\mathcal{M}_{\leq 0}$. For this, we introduce the following notation.

Notation 5.1. Let $\mathcal{M}_{r}$ be the regular locus, i.e. the moduli space of pairs $(C, L)$ such that $C$ is smooth and $L$ is transversal, and $\mathcal{M}_{f}$ the simple singularity locus ( $C$ has at worst simple singularities). Both spaces are open subsets of $\mathcal{M}_{\leq 0}$, and we have: $\mathcal{M}_{r} \subset \mathcal{M}_{f} \subset \mathcal{M}_{\leq 0}$. Note also that $\mathcal{M}_{r} \subset \mathcal{M}_{f} \subset \mathcal{M} \cong \mathcal{D} / \Gamma$ (Theorem 4.1).

To describe the stratification of $\mathcal{M}_{\leq 0}$, we make use of the construction of section 4 of the moduli space of degree 5 pairs as the quotient of a bounded symmetric domain by an arithmetic group. We recall the isomorphism $\mathcal{M}(1) \cong(\mathcal{D} / \Gamma)^{*}$ (cf. Theorems 4.1 and 4.2). Since $\mathcal{M}(1)$ and $\mathcal{M}\left(\frac{5}{2}-\epsilon\right)$ are birational having $\mathcal{M}_{f}$ a common open subset (cf. Corollary [3.2), from the $\mathcal{D} / \Gamma$ description of the moduli of pairs we obtain a good understanding of the regular and simple singularities 
strata $\mathcal{M}_{r}$ and $\mathcal{M}_{f}$. The main conclusion, as in the case of simple and unimodal singularities, is that these spaces can be described as complements of arithmetic arrangements of hyperplanes (Theorem [5.6). Additionally, the possible combinations of simple singularities for a nearby fiber can be obtained in an algorithmic way (see \$5.2).

The structure of the strata $\mathcal{M}_{\leq 0}$ corresponding to the non-simple singularities adjacent to $N_{16}$ is obtained by following the birational modifications that transform $\mathcal{M}(1)$ into $\mathcal{M}\left(\frac{5}{2}-\epsilon\right)$. The nature of the birational map $\mathcal{M}(1) \rightarrow \mathcal{M}\left(\frac{5}{2}-\epsilon\right)$ is both explicit and simple. As noted in $\sqrt{3.3}$, the flips occurring at the wall crossings between 1 and $\frac{5}{2}$ are of the simplest type possible: at the critical slope $t \in\left(1, \frac{5}{2}\right)$ the flip replaces a weighted projective subspace in $\mathcal{M}(t-\epsilon)$ by a weighted projective subspace in $\mathcal{M}(t+\epsilon)$ of complementary dimension. Geometrically, the introduced space corresponds to the stratum of a non-simple singularity. To be more precise, the strata of the triangle singularities adjacent to $N_{16}$ (i.e. $Z_{11}, Z_{12}, W_{12}$, and $W_{13}$ ) are introduced in this way (one at a time) at the slopes $\frac{10}{7}, \frac{8}{5}, \frac{5}{3}$, and $\frac{13}{7}$ respectively. The strata of simple elliptic and cusp singularities are introduced by the birational morphism $\mathcal{M}(1+\epsilon) \rightarrow \mathcal{M}(1)$. The center of this birational map is over the boundary of $\mathcal{D} / \Gamma \cong \mathcal{M}$ in the Baily-Borel compactification $(\mathcal{D} / \Gamma)^{*} \cong \mathcal{M}(1)$ (see $\$ 3.4$ and $\$ 4.4$. The structure of these strata can be made quite explicit by invoking Luna's slice theorem. We only note here that the simple elliptic strata (corresponding to $\widetilde{E_{7}}$ and $\widetilde{E_{8}}$ ) are weighted projective bundles over rational curves (type II boundary components) that parameterize the modulus of the corresponding simple-elliptic singularity.

We close by noting the strong arithmetic nature of the stratification of $\mathcal{M}_{\leq 0}$. For the stratum of simple singularities, this is clear (see Theorem 5.6 below). For the simple elliptic and cusp singularities, we have noted in 33.4 (esp. Figure 3) the relation to the Baily-Borel compactification, analogous to what was observed by Brieskorn [5, 6] for triangle singularities. Finally, the introduction of the strata corresponding to the triangle singularities is very similar to the compactification procedure of Looijenga [20] (esp. [20, §10]). This is discussed in $\$ 5.3$ below.

Remark 5.2. It is probably worthwhile to note what is general and what is special about the case of $N_{16}$. The results of section 2 and the connection between the variation of GIT quotients and the deformations with $\mathbb{C}^{*}$-action are quite general. They hold for any degree $d$ cones and even in higher dimensions. The basic facts behind this are: the singularities are naturally stratified by the log canonical threshold, the log canonical threshold is closely related to the GIT stability condition, the cones are the worst singularities and, finally, a basic construction of the versal deformation space is as a slice of an appropriate Hilbert scheme. What is special about $N_{16}$ is the relation to the $K 3$ surfaces. This is due to the fact that $N_{16}$ is a simple elliptic singularity, and as such it shares quite a few common characteristics (esp. of cohomological nature) with the triangle singularities.

5.1. Discriminants. We start by identifying the complements (the discriminants) of the regular and simple singularities locus, $\mathcal{M}_{r}$ and $\mathcal{M}_{f}$ respectively, in $\mathcal{D} / \Gamma$.

Lemma 5.3. Let $(C, L)$ be a degree 5 pair such that $C+L$ has at worst simple singularities, and let $j: M \hookrightarrow \operatorname{Pic}\left(\widetilde{S}_{(C, L)}\right)$ be the $M$-polarization constructed in 
Proposition 4.25. The following conditions are equivalent:

i) the curve $C$ is smooth and the intersection $C \cap L$ is a transversal;

ii) for every $\delta \in \operatorname{Pic}\left(\widetilde{S}_{(C, L)}\right) \backslash j(M)$ we have $\delta . j(h) \neq 0$.

Proof. By construction, the polarization $j(h)$ defines the double cover map $\widetilde{S}_{(C, L)} \rightarrow$ $\mathbb{P}^{2}$ with branch locus the sextic $B=C+L$. It is well known that the singularities of $B$ are in one-to-one correspondence with the irreducible summands of the root sublattice of $\langle j(h)\rangle_{\operatorname{Pic}\left(\widetilde{S}_{(C, L)}\right)}^{\perp}$. Thus, the condition that $C$ is smooth and that the intersection is a transversal is equivalent to saying that there is no $\operatorname{root} \delta \in \operatorname{Pic}\left(\widetilde{S}_{(C, L)}\right)$ orthogonal to $j(h)$ except those coming from $M$.

The previous lemma says that the non-generic pairs $(C, L)$ are detected by the existence of a root $\delta \in \operatorname{Pic}\left(\widetilde{S}_{(C, L)}\right)$ orthogonal to the polarization class $h$. Arithmetically, one distinguishes two possibilities: either $\delta$ is orthogonal to $M$, or not. The next lemma gives a geometric meaning to this division.

Lemma 5.4. Let $(C, L)$ be a pair and $\left(\widetilde{S}_{(C, L)}, j\right)$ the associated $K 3$ surface. Then

i) the quintic $C$ is singular if and only if there exists $\delta \in \Delta\left(\widetilde{S}_{(C, L)}\right) \cap\langle j(h)\rangle^{\perp}$ such that $\delta \notin j(M)$ and $\delta \perp j(M)$;

ii) the intersection $C \cap L$ is not a transversal if and only if there exists $\delta \in$ $\Delta\left(\widetilde{S}_{(C, L)}\right) \cap\langle j(h)\rangle^{\perp}$ such that $\delta \notin j(M)$ and $\delta \not \perp j(M)$.

Proof. The "only if" part follows by construction. Namely, the case when $C$ is singular away from $L$, or $L$ is tangent to $C$, is immediate. The only thing to check is that if $L$ passes through a singular point of $C$, there exist both a root which is orthogonal to $j(M)$, and another which is not. This follows easily from the construction of Proposition 4.25.

Conversely, assume that there exists $\delta \in \Delta\left(\widetilde{S}_{(C, L)}\right) \cap\langle j(h)\rangle^{\perp}$ such that $\delta \notin j(M)$. By the previous lemma, either $C$ is singular, or the intersection $C \cap L$ is degenerate. The claim is that we can distinguish between the two cases based on the fact that either $\delta$ is orthogonal to $j(M)$ or not. Since $\delta \cdot j(h)=0$, the case that $\delta$ is not orthogonal to $j(M)$ implies $\delta . j\left(e_{i}\right) \neq 0$ for some $i$. Thus, the irreducible summand of the root sublattice of $\langle j(h)\rangle_{\operatorname{Pic}\left(\widetilde{S}_{(C, L)}\right)}^{\perp}$ containing $j\left(e_{i}\right)$ is larger than $A_{1}$. Since by construction $j\left(e_{i}\right)$ corresponds to a point of intersection $p \in C \cap L$, we obtain that the intersection at $p$ is not a transversal.

Assume now that there exists a root $\delta \in \operatorname{Pic}\left(\widetilde{S}_{(C, L)}\right)$ such that $\delta \perp j(M)$. Assume $C$ is smooth. We obtain a contradiction as follows. Since all the singularities of $C+L$ come from the intersection $C \cap L$, we must have that the intersection $C \cap L$ is degenerate at some point $p$ and that $\delta$ belongs to the root system corresponding to the singularity at $p$. Since $C$ is smooth, the only geometric possibility is that $L$ is tangent with multiplicity $k \geq 2$ to $C$ in the point $p$. Thus, the singularity at $p$ for $C+L$ is $A_{2 k-1}$, which gives an embedding $A_{2 k-1} \hookrightarrow\langle j(h)\rangle_{\operatorname{Pic}\left(\widetilde{S}_{(C, L)}\right)}^{\perp}$. Without loss of generality, we can assume $j\left(e_{1}\right), \ldots, j\left(e_{k}\right)$ belong to this $A_{2 k-1}$ root system (see Proposition 4.25). By assumption, $\delta$ also belongs to $A_{2 k-1}$. We obtain that $\delta, \ldots, j\left(e_{k}\right)$ span a sublattice of $A_{2 k-1}$ isometric to $A_{1}^{\oplus(k+1)}$. This is not possible since there is no lattice embedding of $A_{1}^{\oplus(k+1)}$ into $A_{2 k-1}$. 
Notation 5.5. Fix a primitive embedding $M \hookrightarrow \Lambda$ (see 4.2 .2 ). We denote $\Delta_{h}:=$ $\{\delta \in \Delta(\Lambda) \mid \delta . h=0\}$ the set of roots orthogonal to the polarization class $h \in M$. We then define a partition $\Delta_{h}(\Lambda)=\Delta_{\infty} \sqcup \Delta_{f}$ by setting

$$
\Delta_{\infty}=\left\{\delta \in \Delta(\Lambda) \mid \delta . h=0 \text { and } \delta . \delta_{i} \neq 0 \text { for some } i=1, \ldots, 5\right\}
$$

and $\Delta_{f}=\{\delta \in \Delta(\Lambda) \mid \delta \perp M\}=\Delta(T)$ respectively. We denote by $H_{\delta} \subset \mathcal{D}$ the hyperplane orthogonal to a root $\delta$; i.e. $H_{\delta}=\{\omega \in \mathbb{P}(\Lambda \otimes \mathbb{C}) \mid \omega \cdot \omega=0, \omega \cdot \bar{\omega}>$ $0, \omega . \delta=0$, and $\omega \perp M\}$. The sets of roots $\Delta_{\infty}$ and $\Delta_{f}$ are stable under the monodromy group $\Gamma$, and they define two arithmetic arrangements of hyperplanes (in the sense of [20]): $\mathcal{H}_{\infty}=\bigcup_{\delta \in \Delta_{\infty}} H_{\delta}$ and $\mathcal{H}_{f}=\bigcup_{\delta \in \Delta_{f}} H_{\delta}$ respectively.

With these preliminaries, we obtain the following result describing the structure of the regular and the simple singularity part in the deformation space of $N_{16}$. This is analogous to the situation for the triangle singularities ([19, Thm. 6.4] and [5]).

Theorem 5.6. With notation as above, we have the isomorphisms:

i) $\mathcal{M}_{r} \cong\left(\mathcal{D} \backslash\left(\mathcal{H}_{\infty} \cup \mathcal{H}_{f}\right)\right) / \Gamma$,

ii) $\mathcal{M}_{f} \cong\left(\mathcal{D} \backslash \mathcal{H}_{\infty}\right) / \Gamma$.

Proof. This follows from Theorem 4.1 and the fact that the period point $\omega \in \mathcal{D} / \Gamma$ associated to the $K 3$ surface $\widetilde{S}_{(C, L)}$ satisfies:

i) $C$ is singular if and only if $\omega \in \mathcal{H}_{f} / \Gamma$;

ii) the intersection $C \cap L$ is not transversal if and only if $\omega \in \mathcal{H}_{\infty} / \Gamma$

(cf. Lemma 5.4).

5.2. The simple singularities locus. One important application of the construction of section 4 is the description of the possible combinations of simple singularities for a nearby fiber in the deformation of $N_{16}$. This type of application was extensively considered in the case of unimodal singularities (e.g. for triangle singularities see [19, §6] and [38]). In our situation, we obtain the following purely arithmetic characterization of the nearby singularities.

Theorem 5.7. A configuration of simple singularities occurs as the singular locus of a fiber in the universal deformation of $N_{16}$ if and only if the root lattice $R$ associated to the configuration satisfies the following property: there exists an overlattice $N$ of $M \oplus R$ such that

i) the composition $M \hookrightarrow M \oplus R \subseteq N$ is primitive;

ii) the root sublattice of $\langle h\rangle \frac{\perp}{N}$ coincides with $R \oplus A_{1}^{\oplus 5}$;

iii) there exists a primitive embedding of $N$ into the $K 3$ lattice $\Lambda$.

Proof. Assume that the given configuration is realized for a nearby fiber. By Pinkham's compactification procedure, this is equivalent to the existence of a degree 5 pair $(C, L)$ such that $C$ has the given combination of simple singularities, and $L$ is transverse to $C$. It is immediate to see that $M \oplus R$ embeds in the $\operatorname{Pic}\left(\widetilde{S}_{(C, L)}\right)$, and its saturation $N:=\operatorname{Sat}_{\operatorname{Pic}\left(\widetilde{S}_{(C, L)}\right)}(M \oplus R)$ satisfies the required properties.

Conversely, assume that there exists a lattice $N$ satisfying the above properties. By iii) and the surjectivity of the period map for the $K 3$ surfaces there exists a $K 3$ surface $S$ with $\operatorname{Pic}(S)=N$. The assumption i) says that $S$ is $M$-polarized. We 
can arrange that the $M$-polarization is normalized (without affecting the conditions i-iii). By the arguments of Proposition 4.31, it follows that $h \in M$ defines a double cover $\pi: S \rightarrow \mathbb{P}^{2}$ with branch locus a reducible sextic $B=C+L$. The singularities of the sextic $B$ are in bijective correspondence with the irreducible summands of root sublattices of $\langle h\rangle_{\operatorname{Pic}(S)}^{\perp}$. From ii), it then follows that $L$ is transverse to $C$ and the configuration of singularities of $C$ is described by $R$.

It is well known that the combinations of singularities that occur in the deformation of a given simple singularity are described by the proper subgraphs of the corresponding Dynkin diagram. From this fact and the previous theorem, it follows immediately that the singularities of a nearby fiber with only simple singularities can be deformed independently.

Corollary 5.8. Assume that a given configuration of simple singularities occurs as the singular locus for a nearby fiber in the deformation of $N_{16}$. Then, any configuration obtained by deforming this configuration also occurs.

A list of combinations with maximal Milnor number (12 or 13) was obtained by Wall [40 by a case-by-case analysis of the degenerations of plane quintics. Such a list can also be obtained in an algorithmic way by applying Theorem 5.7 and the results of Nikulin [27]. Specifically, given a configuration of simple singularities, we consider the associated root lattice $R$ (the direct sum of the corresponding root lattices of type $A-D-E)$. We can check that the configuration occurs in three basic steps:

Step I: Find all the overlattices $N$ of $M \oplus R$. These are classified by the isotropic subgroups of the corresponding discriminant group ([27, Sect. 4]). Step II: Given $N$ from Step I, check the properties i) and ii) of Theorem 5.7. As the embedding $M \subset N$ constructed above is explicit, this is routine. Step III: Check that $N$ can be primitively embedded in $\Lambda$ (see [27, Thm. 3.6.2]).

We illustrate Theorem 5.7 and the above algorithm with two examples.

Example 5.9. The worst $A_{n}$ singularity that occurs for a nearby fiber is $A_{12}$. First, we note that $A_{13}$ does not occur. We apply Theorem 5.7 for $R=A_{13}$. Since $A_{M \oplus R} \cong(\mathbb{Z} / 2 \mathbb{Z})^{4} \times \mathbb{Z} / 14 \mathbb{Z}$, it follows that there is no proper overlattice of $M \oplus R$ satisfying i). Thus, we must have $N=M \oplus R$. The lattice $N$ cannot be embedded into $\Lambda$, since it does not satisfy the necessary condition $l\left(A_{N}\right) \leq(\operatorname{rk}(\Lambda)-\operatorname{rk}(N))$ (here $l\left(A_{N}\right)=5$ ). On the other hand, for $A_{12}$ the embedding of $N:=M \oplus R$ in $\Lambda$ exists by [27, Thm. 3.6.2]. Note also that all the singularities $A_{n}$ with $n \leq 12$ occur (cf. Corollary [5.8).

Example 5.10. We note that typically in Theorem 5.7 we need to consider proper overlattices of $M \oplus R$. For example, the maximal number of nodes that can occur is 10. Let $R=A_{1}^{\oplus 10}$. Since $l\left(A_{M \oplus R}\right)>\operatorname{rk}(\Lambda)-\operatorname{rk}(M \oplus R)$, the lattice $M \oplus R$ cannot be primitively embedded into the $K 3$ lattice $\Lambda$. Thus we have to consider a proper overlattice $N$ of $M \oplus R$. The overlattices of $M \oplus R$ are classified by the isotropic subgroups $H$ (w.r.t. the induced quadratic form $q_{M}$ ) of $A_{M \oplus R} \cong(\mathbb{Z} / 2 \mathbb{Z})^{\oplus 14}$. Since $A_{N} \cong H^{\perp} / H$, we obtain $l(H) \geq 4$. Together with the conditions i-ii, this 
restriction gives (up to permutations) only one possibility for $H$. The resulting overlattice $N$ embeds into $\Lambda$. Geometrically, the divisibility conditions given by taking the overlattice $M \oplus R \subset N$ translate into collinearity conditions for the nodes. Thus, the need to consider the overlattice $N$ can be interpreted as saying that 10 nodes occur only for quintics consisting of 5 generic lines.

5.3. Triangle singularities adjacent to $N_{16}$ and flips. In $\$ 5.1$ we have seen that the birational modifications that occur when passing from $\mathcal{M} \cong \mathcal{D} / \Gamma$ to $\mathcal{M}_{\leq 0}$ occur over the arrangement of hyperplanes $\mathcal{H}_{\infty}$. Here we show that, in fact, the variation of GIT quotients between the slopes 1 and $\frac{5}{2}$ is essentially dictated by the arithmetic properties of the arrangement $\mathcal{H}_{\infty}$. The situation is very similar to that considered by Looijenga [20, §10], but due to the fact that we work in a relative version, we prove less than [20,10.1]. As in 20], the intersections of the various hyperplanes from the arrangement give a natural stratification of $\mathcal{H}_{\infty}$. We show then that the variation of GIT quotients flips the strata of this stratification, starting with the highest codimension one. Geometrically, these birational transformations can be interpreted as removing the equisingular stratum $\Sigma_{\mathcal{T}}^{+} \subset \mathcal{M}_{\leq 0}$ of the triangle singularities of type $\mathcal{T}$ and replacing it by the deformations of negative weight $\Sigma_{\mathcal{T}}^{-} \subset \mathcal{D} / \Gamma$ of the singularities of type $\mathcal{T}$ (N.B. everything is modulo a $\mathbb{C}^{*}$-action).

The situation described above is essentially identical to that for the deformation of triangle singularities considered in Looijenga [20, §10]. However, there is a major difference: we have to distinguish two cases for the intersection of hyperplanes from the arrangement $\mathcal{H}_{\infty}$, either "transversal" or "tangential". The birational transformations that occur in our situation affect only the strata coming from tangential intersections. In other words, we perform only some of the steps in Looijenga's compactification procedure. It is likely that an appropriate modification of Looijenga's construction will select precisely these steps and give the natural fibration $\mathcal{M}\left(\frac{5}{2}-\epsilon\right) \rightarrow \mathcal{M}\left(\frac{5}{2}\right)$ (see the following remark).

Remark 5.11. We noted in the proof of Lemma 2.16 the existence of two natural discriminant divisors $\Sigma_{0}$ and $\Sigma_{1}$ parameterizing the pairs $(C, L)$ with a singular $C$, and the pairs with a non-transversal $C \cap L$ respectively. Additionally, it is easily seen that $\Sigma_{0}$ and $\Sigma_{1}$ are the natural polarizations on the spaces $\mathcal{M}(0)$ and $\mathcal{M}\left(\frac{5}{2}\right)$ (or more precisely they are nef divisors on appropriate birational models that give the Mori fiber structures $\mathcal{M}(\epsilon) \rightarrow \mathcal{M}(0)$ and $\mathcal{M}\left(\frac{5}{2}-\epsilon\right) \rightarrow \mathcal{M}\left(\frac{5}{2}\right)$ respectively). It was noted in Thaddeus [37, (3.4)] that a 1-parameter variation of GIT quotients is equivalent to running a directed minimal model program (MMP). In our situation, this means that we are running an MMP to change the polarization $\Sigma_{0}$ into $\Sigma_{1} 11$ On the other hand, the proper transforms of $\Sigma_{0}$ and $\Sigma_{1}$ in $\mathcal{M}(1) \cong(\mathcal{D} / \Gamma)^{*}$ can be identified with the discriminants $\mathcal{H}_{f} / \Gamma$ and $\mathcal{H}_{\infty} / \Gamma$ respectively (cf. Lemma 5.4). Since Looijenga's construction [20] is essentially the same as running a directed MMP program for the divisor $\mathcal{H}_{\infty} / \Gamma$, we see that Looijenga's construction should coincide with the variation of GIT construction. The technical details to make this identification more precise are beyond the aim of this paper, so we choose to prove only some weaker statements (e.g. Theorem 5.13 below).

\footnotetext{
${ }^{1}$ Since all the birational morphisms occurring between 0 and $\frac{5}{2}$ are small, the divisors $\Sigma_{0}$ and $\Sigma_{1}$ are well defined Weil divisors in $\mathcal{M}(t)$. Moreover, for non-critical $t \in\left(1, \frac{5}{2}\right)$, the normal variety $\mathcal{M}(t)$ is a geometric quotient, in particular $\mathbb{Q}$-factorial. Thus, for such $t, \Sigma_{0}$ and $\Sigma_{1}$ are $\mathbb{Q}$-Cartier.
} 
5.3.1. The stratification of $\mathcal{H}_{\infty}$ and flips. We make the basic observation that we can characterize in arithmetic terms the locus of degree 5 pairs which are stable at $t=1$, but cease to be stable before $t=\frac{5}{2}$. Namely, all such pairs have the property that there exists a point $p$ such that $\operatorname{mult}_{p}(C \cap L) \geq 3$ and the singularity at $p$ of $C+L$ is a simple singularity (cf. Lemma 3.15). This type of condition can be translated into statements about the Picard lattice of $\widetilde{S}_{(C, L)}$ as in $\$ 4.2$.

Definition 5.12. Let $\delta_{1}, \delta_{2} \in \Delta_{\infty}$ be two roots such that the corresponding hyperplanes in $\mathcal{H}_{\infty}$ satisfy $\mathcal{H}_{\delta_{1}} \neq \mathcal{H}_{\delta_{2}}$ and $\mathcal{D} \cap \mathcal{H}_{\delta_{1}} \cap \mathcal{H}_{\delta_{2}} \neq \emptyset$. We say that the intersection of the hyperplanes $\mathcal{H}_{\delta_{1}}$ and $\mathcal{H}_{\delta_{2}}$ is tangential iff $e_{i} . \delta_{j} \neq 0$ for $j=1,2$ and some fixed $i \in\{1, \ldots, 5\}$. Otherwise, we say that the intersection is transversal.

Geometrically, a hyperplane $\mathcal{H}_{\delta}$ parameterizes the pairs $(C, L)$ with $L$ tangent to $C$. The tangential (transversal) intersection of such hyperplanes corresponds to the case when $L$ is inflectional (resp. bitangent) to $C$. As mentioned above, the birational modification $\mathcal{M}(1) \rightarrow \mathcal{M}\left(\frac{5}{2}-\epsilon\right)$ occurs precisely over the codimension 2 locus in $\mathcal{D} / \Gamma$ given by the tangential intersections of hyperplanes from $\mathcal{H}_{\infty}$. The following result gives a finer stratification of this locus in terms of the factorization of the birational map $\mathcal{M}(1) \rightarrow \mathcal{M}\left(\frac{5}{2}-\epsilon\right)$ in a sequence of simple flips.

Theorem 5.13. For $t \in\left\{\frac{10}{7}, \frac{8}{5}, \frac{5}{3}, \frac{7}{4}, \frac{13}{7}, 2, \frac{11}{5}\right\}$, let $\Sigma_{t}$ be the closure in $\mathcal{M} \cong \mathcal{D} / \Gamma$ of the locus of degree 5 pairs $(C, L)$ with the interval of stability $[\alpha, t]$ (for some $\alpha<1)$. Then $\Sigma_{t}$ is an irreducible intersection of hyperplanes from $\mathcal{H}_{\infty}$, which has the property that a generic point $\omega \in \Sigma_{t}$ determines an $M$-polarized K3 surface with Picard lattice isometric to $M_{t}$ as given in Table 6. Additionally, an embedding $\Sigma_{t} \subset \Sigma_{t}^{\prime}$ coresponds to a tower of primitive embeddings $M \hookrightarrow M_{t^{\prime}} \hookrightarrow M_{t} \hookrightarrow \Lambda$ (where $M$ and $\Lambda$ are as in Notation 4.6). The possible inclusions are described by Table 6 .

Proof. By the results of section 3 (esp. Lemma 3.15] and Table 22), the pairs belonging to $\Sigma_{t}$ are characterized by the worst singularity at infinity, i.e. the type of the simple singularity occurring for $C+L$ in the point of maximal multiplicity for the intersection $C \cap L$. The list of possibilities is given in Table 6. By applying the procedure given by Proposition 4.25, we obtain lattice embeddings $M \hookrightarrow M_{t} \subseteq \operatorname{Pic}\left(\widetilde{S}_{(C, L)}\right)$ (an explicit example is computed in Lemma 5.15 below) such that $M \hookrightarrow \operatorname{Pic}\left(\widetilde{S}_{(C, L)}\right)$ is a normalized embedding (cf. Definition 4.24). By arguments similar to those in $\$ 4.2$, one verifies that the embedding $M_{t} \subseteq \operatorname{Pic}\left(\widetilde{S}_{(C, L)}\right)$ is primitive, that generically we have equality, and that all embeddings $M \hookrightarrow M_{t} \hookrightarrow \Lambda$ are conjugate by $\Gamma$ (N.B. $M \hookrightarrow \Lambda$ is assumed fixed). The fact that $\Sigma_{t}$ is an intersection of hyperplanes from $\mathcal{H}_{\infty}$ is equivalent to saying that $M_{t}$ is generated by elements of $M$ and $\operatorname{rank}\left(M_{t}\right)-\operatorname{rank}(M)$ roots orthogonal to $h$. Finally, the statement about the inclusion $\Sigma_{t} \subset \Sigma_{t}^{\prime}$ is a compatibility statement for the construction of Proposition 4.25. This is easily verified by using the obvious embeddings of root lattices corresponding to the singularities at infinity (e.g. $D_{8} \oplus A_{1} \hookrightarrow D_{10}$ ).

Remark 5.14. Note that the embeddings $M \hookrightarrow M^{\prime} \hookrightarrow \Lambda$ determine embeddings $T^{\prime} \hookrightarrow T$ for the transcendental lattices (i.e. the orthogonal complements in $\Lambda$ ), which in turn determine embeddings of bounded symmetric domains $\mathcal{D}^{\prime} \subset \mathcal{D}$. Thus each $\Sigma_{t}$ has the structure of a locally symmetric variety $\mathcal{D}^{\prime} / \Gamma^{\prime}$ for some $\mathcal{D}^{\prime}$ and $\Gamma^{\prime}$ (or more precisely $\mathcal{D}^{\prime} / \Gamma^{\prime} \rightarrow \Sigma_{t} \subset \mathcal{D} / \Gamma$ is a normalization morphism). 
TABLE 6 . The stratification of $\mathcal{H}_{\infty}$

\begin{tabular}{|c|c|c|c|c|c|}
\hline Case & Wall & Sing. at infinity & Picard lattice $M_{t}$ & Codim. & Specialization of \\
\hline \hline$(1)$ & $\frac{10}{7}$ & $D_{10}$ & $T_{2,3,8}$ & 5 & $(2),(3)$ \\
\hline$(2)$ & $\frac{8}{5}$ & $D_{8}+A_{1}$ & $T_{2,4,6}$ & 4 & $(5),(6)$ \\
\hline$(3)$ & $\frac{5}{3}$ & $A_{9}$ & $T_{2,5,5}$ & 4 & $(5)$ \\
\hline$(4)$ & $\frac{7}{4}$ & $E_{7}+2 A_{1}$ & $E_{8} \oplus U$ & 4 & $(6)$ \\
\hline$(5)$ & $\frac{13}{7}$ & $A_{7}+A_{1}$ & $T_{3,4,4}$ & 3 & $(7)$ \\
\hline$(6)$ & 2 & $D_{6}+2 A_{1}$ & $E_{7} \oplus U$ & 3 & $(7)$ \\
\hline$(7)$ & $\frac{11}{5}$ & $A_{5}+2 A_{1}$ & $E_{6} \oplus U$ & 2 & - \\
\hline
\end{tabular}

Lemma 5.15. Let $S$ be a $K 3$ surface such that $\operatorname{Pic}(S) \cong T_{2,3,8}$. Then there exists $h \in \operatorname{Pic}(S)$ a degree two base point free polarization which defines a double cover map $\pi: S \rightarrow \mathbb{P}^{2}$ with branch locus a reducible sextic $B=C+L$ such that

i) $L$ is a line meeting the residual quintic $C$ in a single point $p$;

ii) $p$ is an ordinary double point for $C$.

Conversely, for a sufficiently general degree 5 pair $(C, L)$ satisfying i) and ii), we have $\operatorname{Pic}\left(\widetilde{S}_{(C, L)}\right) \cong T_{2,3,8}$.

Proof. Let $S$ be a $K 3$ surface such that $\operatorname{Pic}(S) \cong T_{2,3,8}$. We claim that: there exists a normalized embedding $j: M \hookrightarrow \operatorname{Pic}(S)$ such that $\langle j(h)\rangle_{\operatorname{Pic}(S)}^{\perp} \cong D_{10}$. Assuming the claim, from 4.3 .1 it follows that $j(h)$ defines a base point free degree 2 polarization. Again from $\$ 4.3 .1$, the resulting double cover $S \rightarrow \mathbb{P}^{2}$ is branched along a reducible sextic $B=C+L$. The condition $\langle j(h)\rangle_{\operatorname{Pic}(S)}^{\perp} \cong D_{10}$ implies that $B$ has a $D_{10}$ singularity at some point $p$. For numerical reasons, $j\left(l^{\prime}\right)$ has to meet the lattice $D_{10}$. It follows that $p \in L$ (note Lemma 4.30). The only geometric possibility is the situation given by i) and ii). To prove the claim on the existence of a normalized embedding, it suffices to produce an embedding $M \hookrightarrow T_{2,3,8}$ such that the sublattice orthogonal to $h$ is $D_{10}$ (cf. Lemma 4.28). This is done below (see (5.2) ).

Conversely, assume that the pair $(C, L)$ satisfies the conditions i) and ii). Since mult $_{p}(C \cap L)=5$ and $p$ is a node for $C$, it follows that $p$ is a singularity of type $D_{10}$ for $C+L$. The surface $\widetilde{S}_{(C, L)}$ is obtained by desingularizing the double cover of $\mathbb{P}^{2}$ branched along $C+L$. It follows that $\operatorname{Pic}\left(\widetilde{S}_{(C, L)}\right)$ contains 10 exceptional curves $e_{1}, \ldots, e_{10} \in \operatorname{Pic}\left(S_{(C, L)}\right)$, which together with the class $l^{\prime}$ span the $T_{2,3,8}$ sublattice:

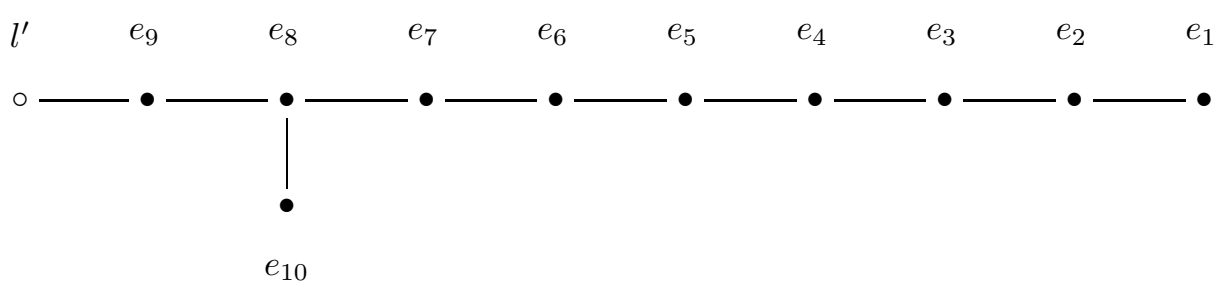


The $M$-polarization $j: M \hookrightarrow \operatorname{Pic}\left(\widetilde{S}_{(C, L)}\right)$ constructed by Proposition 4.25 factors through $T_{2,3,8}$ :

$$
\begin{aligned}
j\left(l^{\prime}\right) & =l^{\prime} \\
j\left(e_{1}\right) & =e_{1}+2\left(e_{2}+\cdots+e_{8}\right)+e_{9}+e_{10} \\
j\left(e_{2}\right) & =e_{3}+2\left(e_{4}+\cdots+e_{8}\right)+e_{9}+e_{10} \\
j\left(e_{3}\right) & =e_{5}+2\left(e_{6}+e_{7}+e_{8}\right)+e_{9}+e_{10} \\
j\left(e_{4}\right) & =e_{7}+2 e_{8}+e_{9}+e_{10} \\
j\left(e_{5}\right) & =e_{9} .
\end{aligned}
$$

The conclusion now follows as in 4.2 .1

Remark 5.16. In principle, it is possible to classify the possible intersection strata of the hyperplanes from $\mathcal{H}_{\infty}$ in purely arithmetic terms. Appropriately considered (e.g. ignore the transversal intersections), the resulting stratification of $\mathcal{H}_{\infty}$ should coincide with the stratification of Theorem 5.13. For triangle singularities, the analogous analysis was done by Looijenga [19, §5]. The arithmetic of our situation is considerably more involved (e.g. the occurrence of high codimension strata).

5.3.2. The geometric interpretation of the flips. We close by taking a closer look at the flips that occur at the walls $\frac{10}{7}, \frac{8}{5}, \frac{5}{3}$, and $\frac{13}{7}$. These are the walls where we introduce strata corresponding to the triangle singularities adjacent to $N_{16}$ into the universal deformation space $\mathcal{M}_{\leq 0}$. We recall the relevant adjacency diagram:

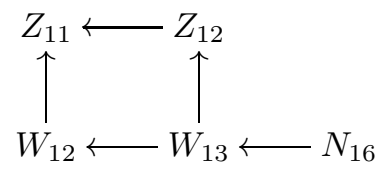

For simplicity, we focus on the first wall $\frac{10}{7}$ and the associated singularity $Z_{11}$. The variation of GIT quotients gives a commutative diagram of birational maps:

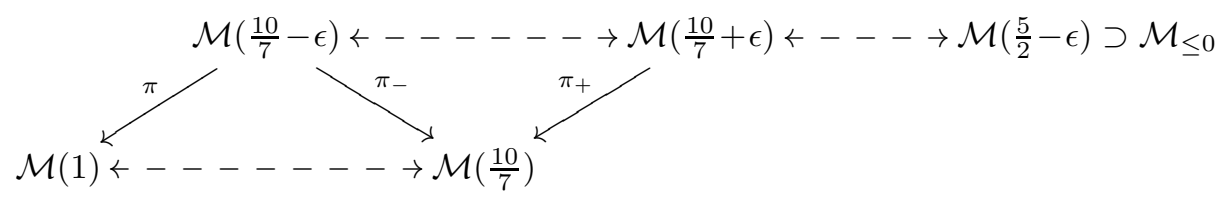

The morphism $\pi$ is a small blow-up of the boundary $\mathcal{D} / \Gamma$ in $(\mathcal{D} / \Gamma)^{*}$ and leaves untouched the stratum $\Sigma_{\frac{10}{7}}$ given by Theorem 5.13. The structure of the morphisms $\pi_{-}$and $\pi_{+}$is very simple (see $\left.§ 3.3\right)$. They contract subspaces $E_{ \pm}$in $\mathcal{M}\left(\frac{10}{7} \pm \epsilon\right.$ ) to a point $z_{0}$ in $\mathcal{M}\left(\frac{10}{7}\right)$ corresponding to the orbit of the pair $\left(C_{0}, L_{0}\right)$, where

$$
C_{0}: x_{0} x_{1} x_{2}^{3}+x_{1}^{5}=0, \quad L_{0}: x_{0}=0
$$

(such a pair is semistable only at $t=\frac{10}{7}$ ). The curve $C_{0}$ has a singularity of type $Z_{11}$ at $x_{0}=(1: 0: 0)$, and $L_{0}$ intersects $C$ with multiplicity 5 in a unique point $p=(0: 0: 1)$. Additionally, the stability condition for degree 5 pairs identifies the two exceptional loci $E_{ \pm}$:

(-) $E_{-}=U_{-} / G$, where $U_{-} \subset X^{s}\left(\frac{10}{7}-\epsilon\right)$ is the $G$-invariant set of pairs $(C, L)$ such that there exists a point $p$ with mult $_{p}(C \cap L)=5$ and $p$ is an ODP for $C$. Furthermore, $C$ has at worst simple, simple elliptic or cusp singularities. 
(+) $E_{+}=U_{+} / G$, where $U_{+} \subset X^{s}\left(\frac{10}{7}+\epsilon\right)$ is the $G$-invariant set of pairs $(C, L)$ such that $C$ has a singularity of type $Z_{11}$. Furthermore, the intersection $C \cap L$ is not too degenerate (better than $L$ being 5 -fold tangent to $C$ in a node).

In particular, it follows that the open stratum $E_{+}^{\circ}$ of $E_{+}$parameterizing pairs with $C \cap L$ a transversal is precisely the $Z_{11}$ equisingular stratum in $\mathcal{M}_{\leq 0}$. Similarly, $E_{-}^{\circ}$ parameterizes pairs with $C$ having simple singularities is the stratum $\Sigma_{\frac{10}{7}} \subset \mathcal{D} / \Gamma$.

It is interesting to note that Luna's slice theorem and the arguments of 22.4 identify $E_{-}$to the quotient $S_{-} / / \operatorname{Aut}\left(C_{0}, L_{0}\right)$, where $S_{-}$denotes the deformations of negative weight of the $Z_{11}$ singularity of $C_{0}$ at $p$. The deformations of negative weight of $Z_{11}$ were studied by Pinkham 31] and Looijenga [19. In particular, they identify the simple singularities locus modulo the $\mathbb{C}^{*}$-action with the moduli space of $T_{2,3,8}$-polarized $K 3$ surfaces. By Lemma 5.15, a $T_{2,3,8}$-polarized $K 3$ surface is $M$-polarized, and the corresponding locus in $\mathcal{D} / \Gamma$ is $\Sigma_{\frac{10}{7}}$. We conclude that the identification $\Sigma_{\frac{10}{7}} \cong E_{-}^{\circ}$ is precisely Pinkham's construction for $Z_{11}$ (N.B. the normalization of $\Sigma_{\frac{10}{7}}$ is isomorphic to $\mathcal{D}^{\prime} / \Gamma^{\prime}$ for appropriate choices for $\mathcal{D}^{\prime}$ and $\Gamma^{\prime}$ ). The role of the lattice $T_{2,3,8}$ for $Z_{11}$ is explained by Pinkham [31, and a posteriori it offers an explanation for the occurrence of $T_{2,3,8}$ in Table 6 ,

The situation for the other triangle singularities adjacent to $N_{16}$ is similar. One only has to note that an adjacency of triangle singularities determines a flip. For example, $Z_{12} \rightarrow Z_{11}$ gives the embedding $\Sigma_{\frac{10}{7}} \subset \Sigma_{\frac{8}{5}}$. To pass from $\Sigma_{\frac{8}{5}} \subset \mathcal{M}(1)$ to $E_{-} \subset \mathcal{M}\left(\frac{8}{5}-\epsilon\right)$, we first need to flip the locus $\Sigma_{\frac{10}{7}}$ (roughly speaking, this introduces the $Z_{11}$ stratum in the deformation of $Z_{12}$ ). This is easily seen to coincide with Looijenga's construction [20, §10] for the triangle singularities. The locus $\Sigma_{\frac{10}{7}} \subset \Sigma_{\frac{8}{5}}$ is precisely the locus of "critical embeddings" of Looijenga [19, $\S 5, \S 6]$. Equivalently, the restriction of the arrangement $\mathcal{H}_{\infty}$ to the subdomain $\mathcal{D}^{\prime \prime}$ corresponding to the singularity $Z_{12}$ (or $\Sigma_{\frac{8}{5}}$ ) is the arrangement considered by Looijenga in [20, 110$]$.

\section{ACKNOWLEDGMent}

This paper is a revised version of the author's thesis. The author is grateful to her advisor, Robert Friedman, for his guidance and help. The author also thanks Michael Thaddeus for teaching her about the variation of GIT quotients, and Igor Dolgachev and Rob Lazarsfeld for helpful comments and suggestions. The referee's comments helped to better organize and clarify the material.

\section{REFERENCES}

1. V. I. Arnol'd, V. V. Goryunov, O. V. Lyashko, and V. A. Vasil'ev, Singularity theory. I, Springer-Verlag, Berlin, 1998. MR1660090(99f:58024)

2. V. I. Arnol'd, S. M. Guseı̌n-Zade, and A. N. Varchenko, Singularities of differentiable maps. Vol. I, Monographs in Mathematics, vol. 82, Birkhäuser, Boston, MA, 1985. MR0777682 (86f:58018)

3. Singularities of differentiable maps. Vol. II, Monographs in Mathematics, vol. 83, Birkhäuser Boston Inc., Boston, MA, 1988. MR0966191 (89g:58024)

4. W. Barth, K. Hulek, C. A. M. Peters, and A. Van de Ven, Compact complex surfaces, second ed., Ergebnisse der Mathematik und ihrer Grenzgebiete. 3. Folge., vol. 4, Springer-Verlag, Berlin, 2004. MR2030225 (2004m:14070) 
5. E. Brieskorn, The unfolding of exceptional singularities, Nova Acta Leopoldina (N.F.) $\mathbf{5 2}$ (1981), no. 240, 65-93, Leopoldina Symposium: Singularities (Thüringen, 1978). MR642697 $(83 \mathrm{k}: 32020)$

6. _ Die Milnorgitter der exzeptionellen unimodularen Singularitäten, Bonner Mathematische Schriften, 150, Universität Bonn Mathematisches Institut, Bonn, 1983. MR733785 (85k:32014)

7. I. V. Dolgachev, Mirror symmetry for lattice polarized K3 surfaces, J. Math. Sci. 81 (1996), no. 3, 2599-2630. MR1420220 (97i:14024)

8. I. V. Dolgachev and Y. Hu, Variation of geometric invariant theory quotients, Inst. Hautes Études Sci. Publ. Math. (1998), no. 87, 5-56. MR1659282(2000b:14060)

9. W. Ebeling, An arithmetic characterisation of the symmetric monodromy groups of singularities, Invent. Math. 77 (1984), no. 1, 85-99. MR751132 (87b:14001)

10. R. Friedman, A new proof of the global Torelli theorem for K3 surfaces, Ann. of Math. (2) 120 (1984), no. 2, 237-269. MR763907 (86k:14028)

11. Algebraic surfaces and holomorphic vector bundles, Universitext, Springer-Verlag, New York, 1998. MR1600388 (99c:14056)

12. P. Hacking, Compact moduli of plane curves, Duke Math. J. 124 (2004), no. 2, 213-257. MR.2078368 (2005f:14056)

13. J. Harris, Galois groups of enumerative problems, Duke Math. J. 46 (1979), no. 4, 685-724. MR.552521 (80m:14038)

14. H. Kim and Y. Lee, Log canonical thresholds of semistable plane curves, Math. Proc. Cambridge Philos. Soc. 137 (2004), no. 2, 273-280. MR2090618(2005m:14055)

15. J. Kollár, Singularities of pairs, Algebraic geometry-Santa Cruz 1995, Proc. Sympos. Pure Math., vol. 62, Amer. Math. Soc., Providence, RI, 1997, pp. 221-287. MR1492525(99m:14033)

16. J. Kollár and S. Mori, Birational geometry of algebraic varieties, Cambridge Tracts in Mathematics, vol. 134, Cambridge University Press, Cambridge, 1998. MR1658959 (2000b:14018)

17. J. Kollár, K. E. Smith, and A. Corti, Rational and nearly rational varieties, Cambridge Studies in Advanced Mathematics, vol. 92, Cambridge University Press, Cambridge, 2004. MR2062787 (2005i:14063)

18. E. Looijenga, On the semi-universal deformation of a simple-elliptic hypersurface singularity. II. The discriminant, Topology 17 (1978), no. 1, 23-40. MR0492380 (58:11503)

19. - The smoothing components of a triangle singularity. II, Math. Ann. 269 (1984), no. 3, 357-387. MR761312 (86d:14033)

20. - Compactifications defined by arrangements. II. Locally symmetric varieties of type $I V$, Duke Math. J. 119 (2003), no. 3, 527-588. MR2003125(2004i:14042b)

21. D. Luna, Adhérences d'orbite et invariants, Invent. Math. 29 (1975), no. 3, 231-238. MR0376704 (51:12879)

22. D. R. Morrison, The Clemens-Schmid exact sequence and applications, Topics in transcendental algebraic geometry, Ann. of Math. Stud., vol. 106, Princeton Univ. Press, 1984, pp. 101119. MR756848

23.

24. S. Mukai, An introduction to invariants and moduli, Cambridge Studies in Advanced Mathematics, vol. 81, Cambridge University Press, Cambridge, 2003. MR 2004218 (2004g:14002)

25. D. Mumford, Stability of projective varieties, Enseignement Math. (2) 23 (1977), no. 1-2, 39-110. MR0450272 (56:8568)

26. D. Mumford, J. Fogarty, and F. Kirwan, Geometric invariant theory, third ed., Ergebnisse der Mathematik und ihrer Grenzgebiete (2), vol. 34, Springer-Verlag, Berlin, 1994. MR 1304906 (95m:14012)

27. V. V. Nikulin, Integral symmetric bilinear forms and some of their applications, Math. USSR Izvestiya 43 (1980), no. 1, 103-167. MR.525944 (80j:10031)

28. H. C. Pinkham, Deformations of algebraic varieties with $G_{m}$ action, Société Mathématique de France, Paris, 1974, Astérisque, No. 20. MR0376672 (51:12847)

29. - Groupe de monodromie des singularités unimodulaires exceptionnelles, C. R. Acad. Sci. Paris Sér. A-B 284 (1977), no. 23, A1515-A1518. MR0439840 (55:12722)

30. Simple elliptic singularities, Del Pezzo surfaces and Cremona transformations, Proc. Sympos. Pure Math., Vol. XXX, Part 1, Amer. Math. Soc., Providence, R. I., 1977, pp. 69-71. MR0441969 (56:358) 
31. Singularités exceptionnelles, la dualité étrange d'Arnold et les surfaces $K-3$, C. R. Acad. Sci. Paris Sér. A-B 284 (1977), no. 11, A615-A618. MR0429876 (55:2886)

32. - Deformations of normal surface singularities with $C^{*}$ action, Math. Ann. 232 (1978), no. 1, 65-84. MR0498543(58:16648)

33. F. Scattone, On the compactification of moduli spaces for algebraic K3 surfaces, Mem. Amer. Math. Soc. 70 (1987), no. 374, x+86. MR912636 (88m:14030)

34. J. Shah, Insignificant limit singularities of surfaces and their mixed Hodge structure, Ann. of Math. (2) 109 (1979), no. 3, 497-536. MR534760(81e:14021)

35. _ A complete moduli space for K3 surfaces of degree 2, Ann. of Math. (2) 112 (1980), no. 3, 485-510. MR.595204 (82j:14030)

36. H. Sterk, Compactifications of the period space of Enriques surfaces. I, Math. Z. 207 (1991), no. 1, 1-36. MR 1106810 (92e:14030)

37. M. Thaddeus, Geometric invariant theory and flips, J. Amer. Math. Soc. 9 (1996), no. 3, 691-723. MR1333296 (96m:14017)

38. T. Urabe, Dynkin graphs, Gabrièlov graphs and triangle singularities, Singularity theory (Liverpool, 1996), London Math. Soc. Lecture Note Ser., vol. 263, Cambridge Univ. Press, Cambridge, 1999, pp. xvii-xviii, 163-174. MR1709351 (2000f:32041)

39. È. B. Vinberg, Some arithmetical discrete groups in Lobačevskǐ spaces, Discrete subgroups of Lie groups and applications to moduli, Oxford Univ. Press, Bombay, 1975, pp. 323-348. MR 0422505 (54:10492)

40. C. T. C. Wall, Highly singular quintic curves, Math. Proc. Cambridge Philos. Soc. 119 (1996), no. 2, 257-277. MR1357043 (97b:14058)

Department of Mathematics, University of Michigan, Ann Arbor, Michigan 48109

E-mail address: rlaza@umich.edu 